\title{
CYCLIC HOMOLOGY AND NONSINGULARITY
}

\author{
JOACHIM CUNTZ AND DANIEL QUILLEN
}

From the pioneering work of Connes [Co1] one knows that periodic cyclic homology can be regarded as a natural extension of de Rham cohomology to the realm of noncommutative geometry. Our aim in this paper is to present the noncommutative analogue of the approach of Deligne [D] and Hartshorne $[\mathrm{H}]$ to de Rham cohomology in algebraic geometry. In this approach de Rham cohomology is first obtained for a nonsingular algebraic variety by means of the de Rham complex of differential forms. An arbitrary variety is then treated by embedding it in a nonsingular variety and completing the de Rham complex of the latter along the subvariety.

In our noncommutative version algebraic varieties are replaced by associative unital algebras over the complex numbers, and nonsingular varieties become algebras which are quasi-free [CQ1]. Indeed, nonsingular varieties are described locally by commutative algebras which behave like free commutative algebras with respect to nilpotent extensions of commutative algebras, while quasi-free algebras are those algebras behaving like free algebras relative to nilpotent algebra extensions. Like a free algebra, a quasi-free algebra $R$ has cohomological dimension $\leq 1$ with respect to Hochschild cohomology, and this implies that its periodic cyclic homology $H P_{\nu} R, \nu \in \mathbb{Z} / 2$, is calculated by the supercomplex

$$
X(R): R \leftrightarrows \Omega^{1} R_{\natural}
$$

discussed for free algebras and coalgebras in [Q1]. This means that $X(R)$ for $R$ quasi-free plays the role in the noncommutative setting of the de Rham complex of a nonsingular variety. Our version of the way de Rham cohomology can be obtained by embedding into a nonsingular variety says that for any algebra extension $A=R / I$ with $R$ quasi-free we have a canonical isomorphism

$$
H P_{*} A=H_{*}\left({\underset{\lim }{n}}_{n} X\left(R / I^{n}\right)\right) .
$$

As an immediate consequence we deduce Goodwillie's theorem [G] that a nilpotent extension $A^{\prime} \rightarrow A$ gives rise to an isomorphism on periodic cyclic homology. In fact, as we show in $\S 10$, our methods yield a refinement of this theorem in which an inverse with respect to cup product for the homomorphism $A^{\prime} \rightarrow A$ is constructed in bivariant periodic cyclic cohomology. For this result to be valid, it is necessary to define bivariant periodic cyclic cohomology in a

Received by the editors October 7, 1993.

1991 Mathematics Subject Classification. Primary 14A22, 58B30.

(C) 1995 American Mathematical Society $0894-0347 / 95 \$ 1.00+\$ .25$ per page 
slightly different way from Jones-Kassel [JK], since the inverse class need not come from $H C^{2 n}\left(A, A^{\prime}\right)$ for large $n$.

In order to extend (2) to include Hochschild and cyclic homology, we consider towers of supercomplexes. On one hand, an ideal $I$ in $R$ gives rise to a decreasing filtration $F_{I}^{p} X(R)$ and a corresponding tower $\mathscr{X}(R, I)$ of quotient supercomplexes of $X(R)$. On the other hand, an algebra $A$ determines a mixed complex $\Omega A$ consisting of noncommutative differential forms with the operators $b$ and $B$, and this in turn gives rise to a tower of supercomplexes $\theta \Omega A$ from which the Hochschild, cyclic, and periodic cyclic homology associated to $A$ can be obtained. Our main result says that for a quasi-free extension $A=R / I$, i.e., $R$ quasi-free, one has a homotopy equivalence of towers

$$
\mathscr{X}(R, I) \sim \theta \Omega A \text {. }
$$

Besides (2) one can derive from (3) the main results of [Q2] in improved form. For instance we find that any class in $H C^{2 n} A$ or $H C^{2 n+1} A$ is represented by a trace or cyclic 1-cocycle respectively on $R / I^{n+1}$. Moreover, when $R$ is free, since any cyclic 1 -cocycle on $R / I^{n+1}$ comes from a trace on $I^{n+1}$ via a connecting homomorphism, we conclude that any class in $H C^{2 n+1} A$ is represented by a trace on $I^{n+1}$.

The proof of (3) breaks into two parts, the first being to construct explicitly the desired homotopy equivalence in the case of the universal extension $A=$ $R A / I A$. In $\S 5$ we identify $X(R A)$ with $\Omega A$ as $\mathbb{Z} / 2$-graded vector spaces and calculate the differential in $X(R A)$ in terms of the canonical operators $d, b, \kappa$ on $\Omega A$ studied in [CQ2]. This differential is similar to $b+B$, and we can relate the two by means of the spectral decomposition associated to the Karoubi operator $\kappa$. The spectral projection associated to the eigenvalue 1 then yields canonical special deformation retractions of both $X(R A)$ and $(\Omega A, b+B)$ onto subcomplexes which are isomorphic. In this way we obtain in $\S 6$ an explicit homotopy equivalence between these two supercomplexes as well as between the towers $\mathscr{X}(R A, I A)$ and $\theta \Omega A$.

This part of the proof can be understood as a variation on the basic theme of representing cyclic cohomology classes on $A$ by traces of the appropriate type on algebras constructed from $A$ such as $\Omega A, R A$, and $Q A$; cf. [Co1], [Co2], [CC], [Cu1], [Cu2]. To be more specific, recall that Connes [Co2] has established an equivalence between supertraces on $Q A$ and $b+B$ cocycles on $A$ fixed under $\kappa$; cf. [CQ2] for this formulation. We in effect prove a similar assertion with $Q A$ replaced by $X(R A)$, namely, that if contributions from the eigenvalues $\neq 1,-1$ of $\kappa$ are ignored, then traces and cyclic 1-cocycles on $R A$ are equivalent to even and odd $b+B$ cocycles on $A$ respectively.

The second part of the proof corresponds to Deligne's result that de Rham cohomology defined by an embedding into a nonsingular variety is independent of the choice of embedding. It involves showing for a quasi-free extension $A=R / I$ that up to homotopy equivalence the tower $\mathscr{X}(R, I)$ depends only on $A$. This is done in $\S \S 8-9$ by following the construction of derived functors, with the extension $A=R / I$ playing the role of a projective resolution of $A$ and the tower $\mathscr{X}(R, I)$ corresponding to a functor applied to this resolution. Given another extension $A=R^{\prime} / I^{\prime}$, we use the lifting property of $R$ with respect to 
nilpotent extensions to construct a homomorphism $u$ of inverse systems of algebras from $\left(R / I^{n+1}\right)$ to $\left(R^{\prime} / I^{\prime n+1}\right)$, which then induces a map $u_{*}$ from $\mathscr{X}(R, I)$ to $\mathscr{X}\left(R^{\prime}, I^{\prime}\right)$. Moreover, two choices for $u$ can be joined by a oneparameter family $u_{t}$ depending on $t$ in a polynomial way. The key point is that $\left(u_{t}\right)_{*}$ modulo (chain) homotopy is independent of $t$, and this is established with the aid of a suitable Cartan homotopy formula for the $X$ complex which is derived in $\S 7$.

This paper is organized as follows. In the first section we recall from [CQ2] how to handle cyclic type homology by means of towers of supercomplexes, and then in $\S 2$ we extend this to include bivariant cyclic cohomology. The third section is an account of the canonical operators on $\Omega A$ and their properties [CQ2], including the spectral decomposition associated to the Karoubi operator. In $\S 4$ we introduce the basic objects $X(R)$ and $\mathscr{X}(R, I)$ and explain the dual interpretation of $X(R)$ in terms of traces and the homotopy relation on traces. The next five sections are devoted to the proof of the main result (3) and some applications, while $\S 10$ contains the refinement of Goodwillie's theorem we have mentioned. $\S 11$ is devoted to a simple construction of Nistor's bivariant Chern character $[\mathrm{N}]$ in our framework.

In $\S 12$ we apply our description (2) to construct the Chern character from $K_{0} A$ and $K_{1} A$ to $H P_{*} A$ by lifting idempotent and invertible matrices in nilpotent extensions. $\S 13$ concerns special features in the case of commutative algebras due to the fact that one has the ordinary de Rham complex $\Omega_{A}$ in addition to $\Omega A$. One of the purposes of these two sections is to make a link with index theory, especially to point out the relation of our work to basic ideas going back to Fedosov's version of the index theorem [F]. In the last section we study the $X$ complex of the tensor product of two algebras, and then apply this to derive a higher homotopy result for the $X$ complex.

Throughout this paper we work over the complex numbers $\mathbb{C}$, however, with minor modifications which will be pointed out at the appropriate places, the arguments are easily seen to hold over an arbitrary groundfield of characteristic zero.

\section{TOWERS OF SUPERCOMPLEXES AND CYCLIC TYPE HOMOLOGY}

The cyclic homology of an algebra $A$ is usually defined with the aid of a suitable mixed complex of chains on the algebra. From the bicomplex associated to the mixed complex one obtains an $S$-module (i.e., a complex equipped with an endomorphism $S$ of degree -2), and the cyclic homology is defined as the homology of this $S$-module. The $S$-module associated to an algebra in this way serves also to define periodic cyclic and negative cyclic homology, as well as bivariant cyclic cohomology [JK] in the cases of two algebras.

For our purposes we require another approach to this cyclic type homology and cohomology which utilizes supercomplexes instead of complexes. Recall that a supercomplex $K$ is a $\mathbb{Z} / 2$-graded vector space equipped with an odd operator $d$ of square zero, and that it has homology groups $H_{\nu} K, \nu \in \mathbb{Z} / 2$. In this paper it will be convenient to write an element of $\mathbb{Z} / 2$ as a coset $n+2 \mathbb{Z}$, and also, depending on the context, to use the standard abbreviations + and - for even and odd respectively. 
As motivation we begin with the periodic cyclic homology arising from a mixed complex. We recall [K1] that a mixed complex $M$ is a $\mathbb{Z}$-graded complex $\bigoplus_{n} M_{n}$, bounded below, whose differential (denoted $b$ by tradition) has degree -1 , which is equipped with an operator $B$ of degree +1 satisfying $[b, B]=$ $B^{2}=0$. The homology of $M$ as a complex, i.e., with respect to $b$, is by definition the Hochschild homology of $M$ :

$$
H H_{n} M=H_{n} M \text {. }
$$

Associated to $M$ is the supercomplex given by

$$
\widehat{M}=\prod_{n} M_{n}
$$

equipped with the usual even-odd grading and the differential $b+B$. The periodic cyclic homology is then the homology of this supercomplex:

$$
H P_{\nu} M=H_{\nu} \widehat{M}, \quad \nu \in \mathbb{Z} / 2
$$

We shall need a similar description involving supercomplexes of the cyclic homology $H C_{n} M$ attached to $M$. As periodic cyclic homology is the inverse limit of cyclic homology in a certain sense, it is reasonable to introduce the following object.

By a tower of supercomplexes, or tower for brevity, we mean an inverse system of supercomplexes $\mathscr{X}=\left(\mathscr{X}^{n}\right)$ indexed by the integers such that the maps $\mathscr{X}^{n} \rightarrow \mathscr{X}^{n-1}$ are all surjective, and such that $\mathscr{X}$ is bounded below in the sense that $\mathscr{X}^{n}=0$ for $n \ll 0$. We associate to $\mathscr{X}$ the filtered supercomplex given by the inverse limit equipped with the induced filtration

$$
\widehat{\mathscr{X}}=\lim \mathscr{X}^{n}, \quad F^{n} \widehat{\mathscr{X}}=\operatorname{Ker}\left(\widehat{\mathscr{X}} \rightarrow \mathscr{X}^{n}\right) \text {. }
$$

As $\widehat{\mathscr{X}} / F^{n} \widehat{\mathscr{X}}=\mathscr{X}^{n}$, the tower can be recovered from this filtered supercomplex. Furthermore, we obtain in this way an equivalence between towers and supercomplexes $K$ equipped with a decreasing filtration $\left(F^{n} K\right)$ by subcomplexes such that $F^{n} K=K$ for $n \ll 0$, and such that $K$ is complete for the topology defined by the filtration.

We call $\mathscr{X}^{n}$ the $n$th level and

$$
\operatorname{gr}^{n} \mathscr{X}=\operatorname{Ker}\left(\mathscr{X}^{n} \rightarrow \mathscr{X}^{n-1}\right)=F^{n-1} \widehat{\mathscr{X}} / F^{n} \widehat{\mathscr{X}}
$$

the $n$th layer of the tower $\mathscr{X}$.

For example, consider the mixed complex $M$ as a supercomplex with the usual even-odd grading and the differential $b+B$. Define the Hodge filtration of $M$ by

$$
F^{n} M=b M_{n+1} \oplus \bigoplus_{k>n} M_{k}
$$

This is a decreasing filtration such that $F^{n} M=M$ for $n \ll 0$. As $F^{n} M$ is closed under $b$ and $B$, it is a subcomplex of $M$, so we obtain a tower

$$
\theta M=\left(M / F^{n} M\right)
$$


which will be called the Hodge tower associated to $M$. We have

$$
\theta \widehat{M}=\lim _{\longleftarrow} M / F^{n} M=\widehat{M}
$$

so the inverse limit of this tower is the supercomplex $\widehat{M}$ giving the periodic cyclic homology of $M$. The $n$th layer is

$$
F^{n-1} M / F^{n} M: M^{n} / b M_{n+1} \underset{b}{\stackrel{0}{\leftrightarrows}} b M_{n}
$$

where $M_{n} / b M_{n+1}$ is in degree $n+2 \mathbb{Z}$. Thus we have

$$
\begin{aligned}
& H_{n+2 \mathbb{Z}}\left(F^{n-1} M / F^{n} M\right)=H_{n} M=H H_{n} M, \\
& H_{n-1+2 \mathbb{Z}}\left(F^{n-1} M / F^{n} M\right)=0
\end{aligned}
$$

so the layers of the Hodge tower give the Hochschild homology of $M$.

The Hodge tower $\theta M$ is an example of a special tower, by which we mean a tower $\mathscr{Z}$ such that the homology of the $n$th layer is supported in degree $n+2 \mathbb{Z}$ for all $n$ :

$$
H_{n-1+2 \mathbb{Z}}\left(\operatorname{gr}^{n} \mathscr{X}\right)=0 \quad \forall n .
$$

Let us define Hochschild, cyclic, and de Rham homology for a special tower $\mathscr{Z}$ by

$$
\begin{aligned}
& H H_{n} \mathscr{X}=H_{n+2 \mathrm{Z}}\left(\mathrm{gr}^{n} \mathscr{X}\right), \\
& H C_{n} \mathscr{X}=H_{n+2 \mathrm{Z}}\left(\mathscr{X}^{n}\right), \\
& H D_{n-1} \mathscr{X}=H_{n-1+2 \mathbb{Z}}\left(\mathscr{X}^{n}\right),
\end{aligned}
$$

respectively. From the short exact sequence of supercomplexes

$$
0 \rightarrow \mathrm{gr}^{n} \mathscr{X} \rightarrow \mathscr{X}^{n} \rightarrow \mathscr{X}^{n-1} \rightarrow 0
$$

we obtain a circular six term exact sequence on passing to homology. By the condition (10) the homology sequence can be written as a five term exact sequence

$$
0 \rightarrow H D_{n-1} \mathscr{X}^{n} \rightarrow H C_{n-1} \mathscr{X} \rightarrow H H_{n} \mathscr{X} \rightarrow H C_{n} \mathscr{X} \rightarrow H D_{n-2} \mathscr{X} \rightarrow 0 .
$$

Splicing these together for different $n$ yields the Connes exact sequence

$$
\rightarrow H C_{n+1} \mathscr{X} \stackrel{s}{\rightarrow} H C_{n-1} \mathscr{X} \rightarrow H H_{n} \mathscr{X} \rightarrow H C_{n} \mathscr{X} \stackrel{s}{\rightarrow} H C_{n-2} \mathscr{X} \rightarrow
$$

where $S: H C_{n} \mathscr{Z} \rightarrow H C_{n-2} \mathscr{Z}$ is the map on homology of degree $n+2 \mathbb{Z}$ induced by the canonical surjection $\mathscr{X}^{n} \rightarrow \mathscr{X}^{n-2}$. In addition we have

$$
H D_{n} \mathscr{X}=\operatorname{Im}\left\{S: H C_{n+2} \mathscr{X} \rightarrow H C_{n} \mathscr{X}\right\}
$$

expressing de Rham homology in terms of cyclic homology. This formula justifies the terminology 'de Rham homology' by virtue of the Connes-Karoubi theorem [Co1, II.33], [Ka, 2.15].

We define periodic cyclic homology for special towers by

$$
H P_{\nu} \mathscr{X}=H_{\nu} \widehat{\mathscr{X}} \text {. }
$$


It is clear from (8) and (9) that the Hodge tower $\theta M$ has the same Hochschild homology and periodic cyclic homology as $M$. Our next task will be to extend this result to cyclic homology, and for this purpose we need to consider $S$ modules [JK], [K2].

An $S$-module is a complex $Q=\bigoplus Q_{n}$ bounded below, with differential $d$ of degree -1 , which is equipped with an operator $S$ of degree -2 commuting with $d$. A mixed complex $M$ determines an $S$-module $\mathscr{B} M$, which is the total complex of the $b, B$ bicomplex of $M$ in the right half plane:

$$
(\mathscr{B} M)_{n}=\bigoplus_{p \geq 0} M_{n-2 p}, \quad d=b+B,
$$

where $S:(\mathscr{B} M)_{n} \rightarrow(\mathscr{B} M)_{n-2}$ is the evident projection killing the summand for $p=0$. By definition the cyclic homology of $M$ is

$$
H C_{n} M=H_{n}(\mathscr{B} M) \text {. }
$$

The $S$-module $\mathscr{B} M$ is an example of a divisible $S$-module, by which we mean that the $S$ operator is surjective. Let $Q$ be any divisible $S$-module $Q$, let ${ }_{S} Q$ be the kernel of $S$ on $Q$, and let

$$
\widehat{Q}_{n+2 \mathbb{Z}}=\lim _{n+2 k}
$$

where the inverse limit is taken with respect to $S$. Then ${ }_{S} Q$ is a complex and $\widehat{Q}$ is a supercomplex. Let the Hochschild, cyclic, and periodic cyclic homology of $Q$ be defined by

$$
\begin{aligned}
& H H_{n} Q=H_{n}\left({ }_{S} Q\right), \\
& H C_{n} Q=H_{n} Q, \\
& H P_{\nu} Q=H_{\nu} \widehat{Q},
\end{aligned}
$$

respectively. In the case $Q=\mathscr{B} M$ we have

$$
{ }_{s}(\mathscr{B} M)=M, \quad \mathscr{B} \widehat{M}=\widehat{M},
$$

so it is clear from the definitions that $\mathscr{B} M$ and $M$ have the same Hochschild, cyclic, and periodic cyclic homology.

By the definition of divisible we have an exact sequence of complexes

$$
0 \rightarrow{ }_{S} Q \rightarrow Q \stackrel{S}{\rightarrow} Q[2] \rightarrow 0 .
$$

On passing to homology we obtain a Connes exact sequence relating the Hochschild and cyclic homology of $Q$. When $Q=\mathscr{B} M$ this is the usual Connes exact sequence for the mixed complex $M$.

We next present a construction going from a divisible $S$-module $Q$ to a special tower, which yields the Hodge tower $\theta M$ in the case of $\mathscr{B} M$.

Let $(\alpha Q)^{n}$ be the supercomplex given by

$$
\left.(\alpha Q)_{n+2 \mathbb{Z}}^{n}=Q_{n} / d{ }_{S} Q_{n+1}\right), \quad(\alpha Q)_{n-1+2 \mathbb{Z}}^{n}=Q_{n-1}
$$

where half of the differential is induced by $d: Q_{n} \rightarrow Q_{n-1}$ and the other half is given by lifting with respect to $S: Q_{n+1} \rightarrow Q_{n-1}$ and then applying $d: Q_{n+1} \rightarrow$ 
$Q_{n}$. We have

$$
\begin{aligned}
& \operatorname{Ker}\left\{d: Q_{n} / d\left({ }_{S} Q_{n+1}\right) \rightarrow Q_{n-1}\right\}=Z_{n} Q / d\left({ }_{S} Q_{n+1}\right), \\
& \operatorname{Im}\left\{d: Q_{n-1} \rightarrow Q_{n} / d\left({ }_{S} Q_{n+1}\right)\right\}=d Q_{n+1} / d\left({ }_{S} Q_{n+1}\right)
\end{aligned}
$$

where $Z_{n} Q$ denotes the space of cycles of degree $n$. Thus

$$
H_{n+2 \mathbb{Z}}(\alpha Q)^{n}=H_{n} Q=H C_{n} Q .
$$

For each $n$ there is a surjection of supercomplexes

$$
\begin{array}{ccc}
(\alpha Q)^{n}: Q_{n} / d\left({ }_{S} Q_{n+1}\right) & \leftrightarrows & Q_{n-1} \\
\downarrow & \downarrow \\
(\alpha Q)^{n-1}: & Q_{n-2} & \leftrightarrows Q_{n-1} / d\left({ }_{S} Q_{n}\right)
\end{array}
$$

induced by $S: Q_{n} \rightarrow Q_{n-2}$ and the identity on $Q_{n-1}$. We thus have a tower $\alpha Q$ consisting of the $(\alpha Q)^{n}$ and these surjections.

Clearly the inverse limit of this tower is the supercomplex $\widehat{Q}$ giving the periodic cyclic homology of $Q$. On the other hand the $n$th layer is

$$
\operatorname{gr}^{n} \alpha Q:{ }_{S} Q_{n} / d\left({ }_{S} Q_{n+1}\right) \underset{\vec{d}}{\stackrel{0}{\leftrightarrows}} d\left({ }_{S} Q_{n}\right),
$$

hence

$$
\left.H_{n+2 \mathbb{Z}}\left(\operatorname{gr}^{n} \alpha Q\right)=H_{n}{ }_{S} Q\right)=H H_{n} Q, \quad H_{n-1+2 \mathbb{Z}}\left(\operatorname{gr}^{n} \alpha Q\right)=0 .
$$

Thus we conclude that $\alpha Q$ is a special tower having the same Hochschild, cyclic, and periodic cyclic homology as $Q$.

Finally we observe that when $Q=\mathscr{B} M$, then $\alpha Q$ is the Hodge tower of $M$, i.e., there is a canonical isomorphism

$$
\theta M=\alpha \mathscr{B} M \text {. }
$$

Indeed, in this case ${ }_{s} Q$ is $M$ with the differential $b$, so $d\left({ }_{s} Q_{n+1}\right)=b M_{n+1}$ and

$$
(\alpha Q)^{n}=\left(\bigoplus_{p \geq 0} M_{n-2 p}\right) / b M_{n+1} \oplus \bigoplus_{p \geq 0} M_{n-1-2 p}=M / F^{n} M
$$

as claimed. Therefore we have established that the Hodge tower $\theta M$ has the same Hochschild, cyclic, and periodic cyclic homology as $M$.

For each of the three types of algebraic objects we have been discussing, namely, mixed complexes, $S$-modules, and towers, there is a natural notion of map respecting the structure. Thus we have the following categories:

$\mathscr{C}_{\Lambda}$ : the category of mixed complexes,

$\mathscr{C}_{S}:$ the category of $S$-modules,

$\mathscr{C}_{S}^{d}:$ the full subcategory of divisible $S$-modules,

$\mathscr{T}^{S}$ : the category of towers,

$\mathscr{T}^{s}:$ the full subcategory of special towers. 
Moreover $\mathscr{B}$ and $\alpha$ are functors

$$
\mathscr{C}_{\Lambda} \stackrel{\mathscr{B}}{\rightarrow} \mathscr{C}_{S}^{d} \stackrel{\alpha}{\rightarrow} \mathscr{T}^{s}
$$

and we have Hochschild, cyclic, and periodic cyclic homology functors from each of these three categories to vector spaces. The main point of the above discussion, apart from all the definitions, is the following.

Proposition 1.1. The functors $\mathscr{B}, \alpha$, and their composition $\theta$, which sends a mixed complex to its Hodge tower, are compatible up to canonical isomorphism with Hochschild, cyclic, and periodic cyclic homology.

We record for later reference the following formulas which we have established for various homology groups attached to the Hodge tower $\theta M$ :

$$
\begin{aligned}
H_{\nu}\left(F^{n-1} M / F^{n} M\right) & = \begin{cases}H H_{n} M, & \nu=n+2 \mathbb{Z}, \\
0, & \nu=n-1+2 \mathbb{Z},\end{cases} \\
H_{\nu}\left(M / F^{n} M\right) & = \begin{cases}H C_{n} M, & \nu=n+2 \mathbb{Z}, \\
H D_{n-1} M, & \nu=n-1+2 \mathbb{Z},\end{cases} \\
H_{\nu} \widehat{M} & =H P_{\nu} M .
\end{aligned}
$$

Remarks. (1) It is worth mentioning for the sake of completeness the following addition to the above formulas:

$$
H_{\nu}\left(F^{n-1} \widehat{M}\right)= \begin{cases}H C_{n}^{-} M, & \nu=n+2 \mathbb{Z}, \\ S\left(H C_{n+1}^{-} M\right), & \nu=n-1+2 \mathbb{Z},\end{cases}
$$

involving the negative cyclic homology; cf. [Q3, §4 (15)].

(2) So far we have considered $M / F^{n} M$ only as a supercomplex, but it is in fact a quotient mixed complex of $M$ having the same Hochschild homology in degrees $\leq n$ and zero Hochschild homology in degrees $>n$. The inverse system of these quotient mixed complexes can be viewed as a kind of Postnikov system, where $M / F^{n} M$ is the $n$th order approximation to the cyclic homology type of the mixed complex $M$. When the Hochschild homology vanishes in degrees $>n$, this $n$th order approximation is exact in the sense that the canonical surjection $M \rightarrow M / F^{n} M$ induces an isomorphism on the cyclic type homology associated to these mixed complexes. In particular, the periodic cyclic homology is given by the supercomplex $M / F^{n} M$.

We turn next to the mixed complexes which will be important later.

Let $A$ be an (associative unital) algebra, and let $\Omega=\Omega A$ be the graded vector space of noncommutative differential forms on $A$. Then $\Omega$ is a mixed complex in a canonical way; we refer to $\S 3$ for the operators $b, B$, and to 3.1(f) for the basic identity $b B+B b=0$. The cyclic type homology of the algebra $A: H H_{n} A, H C_{n} A, H P_{\nu} A$, etc. may be defined as the cyclic type homology arising from this mixed complex. In fact $\Omega$ is the smallest of three 'standard' mixed complexes which yield the cyclic type homology of $A$. The $S$-modules corresponding to these mixed complexes are usually denoted $C C(A), \mathscr{B}(A)$, and $\mathscr{\mathscr { B }}(A)$ as in [L, 2.1], and $\overline{\mathscr{B}}(A)$ is the $S$-module corresponding to $\Omega$. Explicit homotopy equivalences between these $S$-modules are constructed in [K3]. 
From $\Omega$ and its Hodge filtration $F^{n} \Omega$ we can construct various supercomplexes with the differential $b+B$. We now describe the homology of some of these supercomplexes.

For instance we have from (27) the following homology associated to the Hodge tower $\theta \boldsymbol{\Omega}$ :

$$
\begin{aligned}
H_{\nu}\left(F^{n-1} \Omega / F^{n} \Omega\right) & = \begin{cases}H H_{n} A, & \nu=n+2 \mathbb{Z}, \\
0, & \nu=n-1+2 \mathbb{Z},\end{cases} \\
H_{\nu}\left(\Omega / F^{n} \Omega\right) & = \begin{cases}H C_{n} A, & \nu=n+2 \mathbb{Z}, \\
H D_{n-1} A, & \nu=n-1+2 \mathbb{Z},\end{cases} \\
H_{\nu} \widehat{\Omega} & =H P_{\nu} A .
\end{aligned}
$$

We next consider the homology of $F^{n} \Omega$ with respect to $b+B$. This will involve the reduced cyclic type homology of $A: \bar{H} H_{n} A, \bar{H} C_{n} A$, etc. which is defined by means of the mixed complex $\bar{\Omega}=\Omega / \mathbb{C}$; cf. [LQ, §4].

The following is proved in [CQ2] and an independent proof will be given below in $\S 6$.

Proposition 1.2. The inclusion $\mathbb{C} \subset \Omega$ induces an isomorphism on homology with respect to the differential $b+B$, so that $H_{+}(\Omega)=\mathbb{C}, H_{-}(\Omega)=0$. Equivalently, $\bar{\Omega}$ is acyclic with respect to $b+B$.

As $\bar{\Omega}$ is acyclic, we have

$$
H_{\nu-1}\left(F^{n} \bar{\Omega}\right)=H_{\nu}\left(\bar{\Omega} / F^{n} \bar{\Omega}\right)= \begin{cases}\bar{H} C_{n} A, & \nu=n+2 \mathbb{Z}, \\ \bar{H} D_{n-1} A, & \nu=n-1+2 \mathbb{Z},\end{cases}
$$

where we have used the analogue of $(29)$ for the mixed complex $\bar{\Omega}$.

We now compute the homology of $F^{n} \Omega$ with respect to $b+B$.

First note that if $n<0$, then $F^{n} \Omega=\Omega$ and the homology is given by 1.2.

Next, if $n \geq 1$, then the surjection $F^{n} \Omega \rightarrow F^{n} \bar{\Omega}$ is an isomorphism, so the homology of $F^{n} \Omega$ is given by (30).

Finally, if $n=0$, then $\Omega / F^{0} \Omega$ is $A_{\natural}$ concentrated in even degree, so from the exact sequence

$$
0 \rightarrow F^{0} \Omega \rightarrow \Omega \rightarrow \Omega / F^{0} \Omega \rightarrow 0
$$

we obtain the exact sequence

$$
0 \rightarrow H_{+}\left(F^{0} \Omega\right) \rightarrow \mathbb{C} \rightarrow A_{\natural} \rightarrow H_{-}\left(F^{0} \Omega\right) \rightarrow 0
$$

on passing to homology. Using this we find $H_{-}\left(F^{0} \Omega\right)=\bar{H} C_{0} A$, and $H_{-}\left(F^{0} \Omega\right)$ $=0$ or $\mathbb{C}$ according to whether $1 \notin[A, A]$ holds or not.

In particular we have the formula

$$
H_{n-1+2 \mathbb{Z}}\left(F^{n} \Omega\right)=\bar{H} C_{n} A, \quad n \geq 0 .
$$

\section{BIVARIANT CYCLIC COHOMOLOGY}

Our aim in this section is to include bivariant cyclic cohomology in the picture presented in $\S 1$. We begin with divisible $S$-modules, where bivariant cyclic cohomology may be defined as the cohomology of a suitable mapping complex. 
Let $Q, Q^{\prime}$ be divisible $S$-modules. Let $\operatorname{Hom}_{S}\left(Q, Q^{\prime}\right)$ be the mapping complex such that

$$
\operatorname{Hom}_{S}^{k}\left(Q, Q^{\prime}\right)=\operatorname{Hom}_{S}\left(Q, Q^{\prime}\right)_{-k}
$$

consists of operators $f: Q \rightarrow Q^{\prime}$ of degree $-k$ commuting with $S$, where the differential is $f \mapsto[d, f]$. We define bivariant cyclic cohomology for divisible $S$-modules by

$$
H C^{k}\left(Q, Q^{\prime}\right)=H^{k}\left(\operatorname{Hom}_{S}\left(Q, Q^{\prime}\right)\right)
$$

For three divisible $S$-modules there is a cup product

$$
H C^{j}\left(Q^{\prime}, Q^{\prime \prime}\right) \otimes H C^{k}\left(Q, Q^{\prime}\right) \rightarrow H C^{j+k}\left(Q, Q^{\prime \prime}\right)
$$

induced by the pairing

$$
\operatorname{Hom}_{S}\left(Q^{\prime}, Q^{\prime \prime}\right) \otimes \operatorname{Hom}_{S}\left(Q, Q^{\prime}\right) \rightarrow \operatorname{Hom}_{S}\left(Q, Q^{\prime \prime}\right)
$$

given by composing operators.

According to [JK, 2.2, 5.1], when we pull back this bivariant cyclic cohomology and cup product via $M \mapsto \mathscr{B} M$, we obtain the Jones-Kassel bivariant cyclic cohomology for mixed complexes

$$
H C^{k}\left(M, M^{\prime}\right)=H C^{k}\left(\mathscr{B} M, \mathscr{B} M^{\prime}\right)
$$

and its cup product.

It is useful to interpret the structure on divisible $S$-modules, which is provided by bivariant cyclic cohomology equipped with the cup product operation, in terms of a homotopy category. Note that a map $f: Q \rightarrow Q^{\prime}$ of $S$ modules (i.e., respecting the $S$-module structure) is the same as an element of $Z^{0} \operatorname{Hom}_{S}\left(Q, Q^{\prime}\right)$, where $Z^{0}$ denotes the subspace of elements killed by the differential. We say $f$ is homotopic to zero when $f=[d, h]$ for some $h \in \operatorname{Hom}_{S}^{-1}\left(Q, Q^{\prime}\right)$. Then elements of

$$
H C^{0}\left(Q, Q^{\prime}\right)=Z^{0} \operatorname{Hom}_{S}\left(Q, Q^{\prime}\right) /\left[d, \operatorname{Hom}_{S}^{-1}\left(Q, Q^{\prime}\right)\right]
$$

are homotopy classes of maps of $S$-modules $Q \rightarrow Q^{\prime}$.

Let $H o \mathscr{C}_{S}^{d}$ denote the homotopy category of divisible $S$-modules, in which the morphisms are homotopy classes of maps. Then this homotopy category incorporates the information in $H C^{0}$ together with the cup product.

In order to handle $H C^{k}$ we use the 'suspension' operation $Q \mapsto Q[1]$, whose $k$ th power for any integer $k$ is $Q \mapsto Q[k]$, where

$$
Q[k]_{n}=Q_{n-k}
$$

with $d, S$ on $Q[k]$ given by $(-1)^{k} d, S$ on $Q$. Clearly

$$
\operatorname{Hom}_{S}\left(Q, Q^{\prime}\right)=\operatorname{Hom}_{S}\left(Q[k], Q^{\prime}[k]\right) \text {, }
$$

hence

$$
H C^{0}\left(Q, Q^{\prime}\right)=H C^{0}\left(Q[k], Q^{\prime}[k]\right)
$$

showing that suspension determines an automorphism of the homotopy category $H o \mathscr{C}_{S}^{d}$. 
On the other hand we have

$$
\operatorname{Hom}_{S}^{j}\left(Q, Q^{\prime}[k]\right)=\operatorname{Hom}_{S}^{j+k}\left(Q, Q^{\prime}\right)
$$

where the differential on the left is $(-1)^{k}$ times the differential on the the right, so

$$
H C^{0}\left(Q, Q^{\prime}[k]\right)=H C^{k}\left(Q, Q^{\prime}\right) .
$$

Thus an element of $H C^{k}\left(Q, Q^{\prime}\right)$ can be identified with a map $Q \rightarrow Q^{\prime}[k]$ in $H o \mathscr{C}_{S}^{d}$. With respect to this identification the cup product is easily seen to be

$$
\left(g: Q^{\prime} \rightarrow Q^{\prime \prime}[j]\right)\left(f: Q \rightarrow Q^{\prime}[k]\right)=\left(g[k] \cdot f: Q \rightarrow Q^{\prime \prime}[j+k]\right) .
$$

In this way we can recover bivariant cyclic cohomology for divisible $S$-modules and its cup product from the homotopy category of divisible $S$-modules and the suspension automorphism.

We next want to treat bivariant cyclic cohomology for special towers, but before doing so it will be convenient to introduce homotopy for maps of towers and the corresponding homotopy category $H o \mathscr{T}$.

Let $\mathscr{X}, \mathscr{X}^{\prime}$ be arbitrary towers (of supercomplexes) and let

$$
\operatorname{Hom}_{c}\left(\widehat{\mathscr{X}}, \widehat{\mathscr{X}}^{\prime}\right)=\left\{f: \widehat{\mathscr{X}} \rightarrow \widehat{\mathscr{X}}^{\prime} \mid \forall m \exists n, f\left(F^{n} \widehat{\mathscr{X}}\right) \subset F^{m} \widehat{\mathscr{X}}^{\prime}\right\}
$$

be the supercomplex of continuous linear maps with respect to the natural topologies. Sitting inside this is the subcomplex

$$
\operatorname{Hom}^{k}\left(\mathscr{X}, \mathscr{X}^{\prime}\right)=\left\{f: \widehat{\mathscr{X}} \rightarrow \widehat{\mathscr{X}}^{\prime} \mid \forall m, f\left(F^{m+k} \widehat{\mathscr{X}}\right) \subset F^{m} \widehat{\mathscr{X}}^{\prime}\right\}
$$

of maps of order $\leq k$. Thus $f: \widehat{\mathscr{X}} \rightarrow \widehat{\mathscr{X}}^{\prime}$ has order $\leq k$ iff it induces maps of the quotient spaces $\mathscr{X}^{m+k} \rightarrow \mathscr{X}^{\prime m}$ for all $m$. In this way an element of $\operatorname{Hom}^{k}\left(\mathscr{X}, \mathscr{X}^{\prime}\right)$ can be identified with a map of inverse systems $\left(\mathscr{X}^{n}\right) \rightarrow$ $\left(\mathscr{X}^{\prime n-k}\right)$, which is linear but might not respect the supercomplex structure.

Consequently, a map $\mathscr{X} \rightarrow \mathscr{X}^{\prime}$ in $\mathscr{T}$. (i.e., respecting the supercomplex structure) can be identified with an element $f \in Z_{+} \operatorname{Hom}^{0}\left(\mathscr{X}, \mathscr{X}^{\prime}\right)$, where $Z_{+}$denotes the subspace of even cycles. We say that $f$ is homotopic to zero when $f=[d, h]$ for some $h \in \operatorname{Hom}^{0}\left(\mathscr{X}, \mathscr{X}^{\prime}\right)_{-}$. Then elements of the set $H_{+}\left(\operatorname{Hom}^{0}\left(\mathscr{X}, \mathscr{X}^{\prime}\right)\right)$ are homotopy classes of maps from $\mathscr{X}$ to $\mathscr{X}^{\prime}$, and we have the homotopy category of towers $H o \mathscr{T}$, in which homotopy classes are the morphisms. It should be clear what is meant by a homotopy equivalence of towers, and in particular, by a contractible tower.

We now restrict attention to special towers and let $H o \mathscr{T}^{s} \subset H o \mathscr{T}$ be the full subcategory consisting of special towers. We define bivariant cyclic cohomology for special towers by

$$
H C^{k}\left(\mathscr{X}, \mathscr{X}^{\prime}\right)=H_{k+2 \mathbb{Z}}\left(\operatorname{Hom}^{k}\left(\mathscr{X}, \mathscr{X}^{\prime}\right)\right) .
$$

In the case of three special towers we have a cup product on $H C^{*}$ induced by the pairing

$$
\operatorname{Hom}^{j}\left(\mathscr{X}^{\prime}, \mathscr{X}^{\prime \prime}\right) \otimes \operatorname{Hom}^{k}\left(\mathscr{X}, \mathscr{X}^{\prime}\right) \rightarrow \operatorname{Hom}^{j+k}\left(\mathscr{X}, \mathscr{X}^{\prime \prime}\right)
$$


given by composition. We note that $H C^{0}\left(\mathscr{X}, \mathscr{X}^{\prime}\right)$ is the set of maps $\mathscr{Z} \rightarrow \mathscr{X}^{\prime}$ in the homotopy category $H o \mathscr{T}^{s}$ and the cup product on $H C^{0}$ is composition of maps in this category.

We define suspension $\mathscr{X} \mapsto \mathscr{X}[1]$ for special towers so that its $k$ th power is

$$
\mathscr{X}[k]_{\nu}^{n}=\mathscr{X}_{\nu-k}^{n-k}
$$

with $d$ on the left given by $(-1)^{k} d$ on the right. We have

$$
\begin{aligned}
\operatorname{Hom}^{j}\left(\mathscr{X}[k], \mathscr{X}^{\prime}[k]\right) & =\operatorname{Hom}^{j}\left(\mathscr{X}, \mathscr{X}^{\prime}\right), \\
H C^{0}\left(\mathscr{X}[k], \mathscr{X}^{\prime}[k]\right) & =H C^{0}\left(\mathscr{X}, \mathscr{X}^{\prime}\right)
\end{aligned}
$$

so suspension determines an automorphism of $\mathrm{HoT}^{s}$. It is clear that

$$
\operatorname{Hom}^{p}\left(\mathscr{X}, \mathscr{X}^{\prime}[k]\right)_{\nu}=\operatorname{Hom}^{p+k}\left(\mathscr{X}, \mathscr{X}^{\prime}\right)_{\nu+k}
$$

with $d$ on the left corresponding to $(-1)^{k} d$ on the right, so

$$
H C^{0}\left(\mathscr{X}, \mathscr{X}^{\prime}[k]\right)=H C^{k}\left(\mathscr{X}, \mathscr{X}^{\prime}\right) \text {. }
$$

Thus, as in the case of divisible $S$-modules, we conclude that $H C^{*}$ for special towers together with its cup product operation can be recovered from $H o \mathscr{T}^{s}$ and the suspension automorphism.

We next examine the behavior of the functor $\alpha$ with respect to bivariant cyclic cohomology. Let $Q, Q^{\prime}$ be divisible $S$-modules and $\mathscr{Z}=\alpha Q, \mathscr{Z}^{\prime}=\alpha Q^{\prime}$ the corresponding special towers. We are going to compare

$$
\begin{aligned}
H C^{0}\left(Q, Q^{\prime}\right) & =Z^{0} \operatorname{Hom}_{S}\left(Q, Q^{\prime}\right) /\left[d, \operatorname{Hom}_{S}^{-1}\left(Q, Q^{\prime}\right)\right], \\
H C^{0}\left(\mathscr{X}, \mathscr{X}^{\prime}\right) & =Z_{+} \operatorname{Hom}^{0}\left(\mathscr{X}, \mathscr{X}^{\prime}\right) /\left[d, \operatorname{Hom}^{0}\left(\mathscr{X}, \mathscr{X}^{\prime}\right)_{-}\right] .
\end{aligned}
$$

We have seen that $\widehat{Q}=\widehat{\mathscr{X}}$, and similarly with primes. Recall that $\operatorname{Hom}^{k}\left(\mathscr{X}, \mathscr{X}^{\prime}\right)$ is a subcomplex of the supercomplex

$$
\operatorname{Hom}_{c}\left(\widehat{Q}, \widehat{Q}^{\prime}\right)=\operatorname{Hom}_{c}\left(\widehat{\mathscr{X}}, \widehat{\mathscr{X}}^{\prime}\right)
$$

by definition. On the other hand, because $Q$ is divisible, $Q_{n}$ is a quotient space of $\widehat{Q}_{n+2 Z}$, and similarly for $Q^{\prime}$. Moreover, an element of $\operatorname{Hom}_{S}^{k}\left(Q, Q^{\prime}\right)$ can be identified with a map $f: \widehat{Q} \rightarrow \widehat{Q}^{\prime}$ of degree $k+2 \mathbb{Z}$, which induces maps $Q_{n} \rightarrow Q_{n-k}^{\prime}$ for all $n$. Thus we can regard both $\operatorname{Hom}_{S}^{k}\left(Q, Q^{\prime}\right)$ and $\operatorname{Hom}^{k}\left(\mathscr{X}, \mathscr{X}^{\prime}\right)$ as subspaces of the supercomplex (13). Note that the differential $f \mapsto[d, f]$ on (13) restricts to the differential on the complex $\operatorname{Hom}_{S}\left(Q, Q^{\prime}\right)$ in an evident sense.

With this understood we have the inclusion

$$
\operatorname{Hom}^{0}\left(\mathscr{X}, \mathscr{X}^{\prime}\right)_{+} \subset \operatorname{Hom}_{S}^{0}\left(Q, Q^{\prime}\right) \text {. }
$$

Indeed, the latter consists of $f: \widehat{Q} \rightarrow \widehat{Q}^{\prime}$ which induce maps $Q_{n} \rightarrow Q_{n}^{\prime}$ for all $n$, while the former consists of such $f$ which in addition induce maps from 
$Q_{n} / d\left({ }_{s} Q_{n+1}\right)$ to $Q_{n}^{\prime} / d\left({ }_{S} Q_{n+1}^{\prime}\right)$. Notice that when $f$ commutes with $d$ this additional condition is automatic, hence we have

$$
Z_{+} \operatorname{Hom}^{0}\left(\mathscr{X}, \mathscr{X}^{\prime}\right)=Z^{0} \operatorname{Hom}_{S}\left(Q, Q^{\prime}\right) \text {. }
$$

Lemma 2.1. $\operatorname{Hom}^{0}\left(\mathscr{X}, \mathscr{X}^{\prime}\right)_{-}=\operatorname{Hom}_{S}^{-1}\left(Q, Q^{\prime}\right)+\left[d, \operatorname{Hom}^{0}\left(\mathscr{X}, \mathscr{X}^{\prime}\right)_{-}\right]$.

Proof. First of all we have

$$
\operatorname{Hom}^{0}\left(\mathscr{X}, \mathscr{X}^{\prime}\right)_{-} \supset \operatorname{Hom}_{S}^{-1}\left(Q, Q^{\prime}\right)
$$

since if $f: \widehat{Q} \rightarrow \widehat{Q}^{\prime}$ induces $Q_{n} \rightarrow Q_{n+1}^{\prime}$ for all $n$, then it induces $Q_{n-1} \rightarrow$ $Q_{n}^{\prime} / d\left({ }_{S} Q_{n+1}^{\prime}\right)$ and $Q_{n} / d\left({ }_{s} Q_{n+1}\right) \rightarrow Q_{n-2} \rightarrow Q_{n-1}^{\prime}$. Thus the inclusion $\supset$ holds between the subspaces cited in the lemma.

To show the opposite inclusion, let $f \in \operatorname{Hom}^{0}\left(\mathscr{X}, \mathscr{X}^{\prime}\right)_{-}$. Write $\overline{\mathscr{X}}^{n}$ for $\operatorname{gr}^{n} \mathscr{X}$ and similarly with primes, and consider the induced map $\bar{f}^{n}: \overline{\mathscr{X}}^{n} \rightarrow$ $\overline{\mathscr{X}}^{\prime n}$. Since $d: \overline{\mathscr{X}}_{n+2 \mathbb{Z}}^{\prime n} \rightarrow \overline{\mathscr{X}}_{n-1+2 \mathbb{Z}}^{n}$ is surjective, we can choose $\bar{g}^{n}: \overline{\mathscr{X}}_{n+2 \mathbb{Z}}^{n} \rightarrow$ $\overline{\mathscr{X}}_{n+2 \mathbb{Z}}^{\prime n}$ such that $d \bar{g}^{n}=\bar{f}^{n}$ on $\overline{\mathscr{X}}_{n+2 \mathbb{Z}}^{n}$. Extending $\bar{g}^{n}$ to an even map $\overline{\mathscr{X}}^{n} \rightarrow \overline{\mathscr{X}}^{n}$ vanishing on $\overline{\mathscr{X}}_{n-1+2 \mathbb{Z}}^{n}$, we then have that $\bar{f}^{n}$ and $\left[d, \bar{g}^{n}\right]$ agree on $\overline{\mathscr{X}}_{n+2 \mathbb{Z}}^{n}$. We choose $\bar{g}^{n}$ in this way for each $n$ and then choose $g \in$ $\operatorname{Hom}^{0}(\mathscr{X}, \mathscr{Z})_{+}$inducing $\bar{g}^{n}$ on the $n$th layer for all $n$. This is possible because we are working over a field, hence if the differentials are ignored we can suppose the towers are split, i.e.,

$$
\mathscr{X}^{n}=\bigoplus_{m \leq n} \overline{\mathscr{X}}^{m}
$$

and similarly for $\mathscr{X}^{\prime}$. If we put $f^{\prime}=f-[d, g]$, then $f^{\prime}$ kills $\overline{\mathscr{X}}_{n+2 Z}^{n}=$ $\left.{ }_{s} Q_{n} / d{ }_{s} Q_{n+1}\right)$ for all $n$. Thus the map $\left.Q_{n} / d{ }_{s} Q_{n+1}\right) \rightarrow Q_{n-1}^{\prime}$ induced by $f^{\prime}$ descends to a map $Q_{n-2} \rightarrow Q_{n-1}^{\prime}$ for all $n$, which means that $f^{\prime}$ lies in $\operatorname{Hom}_{S}^{-1}\left(Q, Q^{\prime}\right)$. As $f=f^{\prime}+[d, g]$, this shows the inclusion $\subset$ holds between the subspaces cited in the lemma.

This lemma implies $\left[d, \operatorname{Hom}^{0}\left(\mathscr{X}, \mathscr{Z}^{\prime}\right)_{-}\right]=\left[d, \operatorname{Hom}_{S}^{-1}\left(Q, Q^{\prime}\right)\right]$, which together with (11), (12), (14) yields a canonical isomorphism

$$
H C^{0}\left(Q, Q^{\prime}\right)=H C^{0}\left(\mathscr{X}, \mathscr{X}^{\prime}\right) \text {. }
$$

The isomorphisms (14) and (15) have the following interpretations in category terms. We note that the two sides of (14) can be viewed as Hom sets in the categories $\mathscr{T}^{s}$ and $\mathscr{C}_{S}^{d}$ respectively. We can interpret (14) as saying that $\alpha$ induces isomorphisms

$$
Z^{0} \operatorname{Hom}_{S}\left(Q, Q^{\prime}\right) \stackrel{\sim}{\rightarrow} Z_{+} \operatorname{Hom}^{0}\left(\alpha Q, \alpha Q^{\prime}\right), \quad f \mapsto \alpha(f)
$$

for every pair $Q, Q^{\prime}$, in other words, the functor

$$
\alpha: \mathscr{C}_{S}^{d} \rightarrow \mathscr{T}^{s}
$$


is fully faithful. Similarly (15) means that $\alpha$ induces isomorphisms

$$
\alpha_{*}: H C^{0}\left(Q, Q^{\prime}\right) \stackrel{\sim}{\rightarrow} H C^{0}\left(\alpha Q, \alpha Q^{\prime}\right)
$$

and so we also have a fully faithful functor

$$
\alpha: H o \mathscr{C}_{S}^{d} \rightarrow H o \mathscr{T}^{s}
$$

on the level of homotopy categories.

We have now established the degree zero part of the following.

Proposition 2.2. One has canonical isomorphisms

$$
\alpha_{*}: H C^{k}\left(Q, Q^{\prime}\right) \stackrel{\sim}{\rightarrow} H C^{k}\left(\alpha Q, \alpha Q^{\prime}\right)
$$

compatible with cup product.

The isomorphism $\alpha_{*}$ for arbitrary $k$ is obtained as follows:

$$
\begin{aligned}
H C^{k}\left(Q, Q^{\prime}\right) & =H C^{0}\left(Q, Q^{\prime}[k]\right) \\
& =H C^{0}\left(\alpha Q, \alpha\left(Q^{\prime}[k]\right)\right) \\
& =H C^{0}\left(\alpha Q,\left(\alpha Q^{\prime}\right)[k]\right) \\
& =H C^{k}\left(\alpha Q, \alpha Q^{\prime}\right)
\end{aligned}
$$

using (3), (10), and the fact that the functor $\alpha$ commutes with the suspension automorphisms in $H o \mathscr{C}_{S}^{d}$ and $H o \mathscr{T}^{s}$. Thus $\alpha_{*}$ sends the element of $H C^{k}\left(Q, Q^{\prime}\right)$ given by a map $f: Q \rightarrow Q^{\prime}[k]$ in $H o \mathscr{C}_{S}^{d}$ to the element of $H C^{k}\left(\alpha Q, \alpha Q^{\prime}\right)$ given by the map $\alpha(f): \alpha Q \rightarrow \alpha\left(Q^{\prime}[k]\right)=\left(\alpha Q^{\prime}\right)[k]$ in $H o \mathscr{T}^{s}$. It follows easily using (4) that $\alpha_{*}$ is compatible with cup product.

Having treated divisible $S$-modules and special towers, it remains to consider mixed complexes and the Jones-Kassel bivariant cyclic cohomology (2). Again we introduce a category to handle the structure on mixed complexes given by this cohomology and its cup product.

Let us define the derived category of mixed complexes $D \mathscr{C}_{\Lambda}$ to be the category having mixed complexes for objects, in which a map $M \rightarrow M^{\prime}$ is an element of $H C^{0}\left(M, M^{\prime}\right)$ and composition is given by the cup product on $H C^{0}$. We then have a tautological functor

$$
D \mathscr{C}_{\Lambda} \rightarrow H o \mathscr{C}_{S}^{d}, \quad M \mapsto \mathscr{B} M
$$

which is fully faithful. We define suspension for mixed complexes by $M[k]_{n}=$ $M_{n-k}$, with $b, B$ on $M[k]$ given by $(-1)^{k} b,(-1)^{k} B$ on $M$. Since $\mathscr{B}(M[k])$ $=(\mathscr{B} M)[k]$, one sees easily that suspension is an automorphism of $D \mathscr{C}_{\Lambda}$, and that elements of $H C^{k}(M, N)$ can be identified with maps $M \rightarrow N[k]$ in this category, with cup product described in a way similar to (4).

Let us use $\mathscr{B}$ to denote the functor (18). Combining these remarks about this functor, 2.2 about the functor $\alpha$ of (17), and 1.1, we obtain the following result summarizing the results of $\S \S 1$ and 2 . 
Theorem 2.3. One has fully faithful functors

$$
D \mathscr{C}_{\Lambda} \stackrel{\mathscr{B}}{\rightarrow} H o \mathscr{C}_{S}^{d} \stackrel{\alpha}{\rightarrow} H o \mathscr{T}^{s}
$$

which are compatible up to canonical isomorphism with Hochschild, cyclic, and periodic cyclic homology, and with bivariant cyclic cohomology.

Remarks. Since cyclic type homology and cohomology are functors on each of the categories $D \mathscr{C}_{\Lambda}, H o \mathscr{C}_{S}^{d}$, and $H o \mathscr{T}^{s}$, it follows that an isomorphism between two objects in any of these categories induces isomorphisms on cyclic type homology and cohomology involving these objects. For instance, an isomorphism $M \simeq M^{\prime}$ in $D \mathscr{C}_{\Lambda}$ gives rise to an isomorphism of functors $H C^{*}(-, M)$ $\simeq H C^{*}\left(-, M^{\prime}\right)$, and the converse even holds by Yoneda's lemma. In this situation $M$ and $M^{\prime}$ are equivalent from the viewpoint of cyclic homology theory, in other words, they have the same cyclic homology type.

It is thus appropriate to interpret $D \mathscr{C}_{\Lambda}, H o \mathscr{C}_{S}^{d} s$, and $H o \mathscr{T}^{s}$ as the categories of cyclic homology types (or cyclic 'motives') arising from mixed complexes, divisible $S$-modules, and special towers respectively. Moreover, as the functors (19) are fully faithful, each category can be viewed as a full subcategory of the following one. It turns out that both functors are equivalences of categories [Q3], so that all cyclic homology types arising from special towers already come from mixed complexes.

Let us define the cyclic homology type of an algebra $A$ to be $\Omega A$ considered as an object of $D \mathscr{C}_{\Lambda}$. Borrowing the terminology 'represent' as in representing a functor, we say that a mixed complex $M$ (resp. divisible $S$-module $Q$, special tower $\mathscr{Z}$ ) represents the cyclic homology type of $A$ when an isomorphism $\Omega A \simeq$ $M$ in $D \mathscr{C}_{\Lambda}$ (resp. $\mathscr{B} \Omega A \simeq Q$ in $H o \mathscr{C}_{S}^{d}, \theta \Omega A \simeq \mathscr{X}$ in $H o \mathscr{T}^{s}$ ) is specified. In this case we can use $M$ (resp. $Q, \mathscr{X}$ ) to calculate cyclic type homology and cohomology involving $A$. For example, if $\mathscr{X}, \mathscr{X}^{\prime}$ represent the cyclic homology types of $A, A^{\prime}$, then we have

$$
H C^{k}\left(A, A^{\prime}\right)=H^{k}\left(\operatorname{Hom}_{S}\left(\mathscr{B} \Omega A, \mathscr{B} \Omega A^{\prime}\right)\right)=H_{k+2 \mathbb{Z}}\left(\operatorname{Hom}^{k}\left(\mathscr{X}, \mathscr{X}^{\prime}\right)\right) .
$$

\section{OPERATORS ON DIFFERENTIAL FORMS}

In this section we review basic facts about (noncommutative) differential forms; for a more thorough treatment see [CQ2].

Let $A$ be an (associative unital) algebra and let $\Omega A=\bigoplus_{n} \Omega^{n} A$ be the DG algebra of differential forms over $A$. One has the following identification of $n$-forms with tensors:

$$
\begin{aligned}
\Omega^{n} A & =A \otimes \bar{A}^{\otimes n}, \\
a_{0} d a_{1} \cdots d a_{n} & \leftrightarrow a_{0} \otimes \cdots \otimes a_{n}
\end{aligned}
$$

where $\bar{A}=A / \mathbb{C}$. Furthermore, $\Omega A$ is the universal DG algebra generated by $A$ in degree zero; cf. [CA1, $\S 1$ ]. As $A$ is fixed in this section, we put $\Omega=\Omega A$, $\Omega^{n}=\Omega^{n} A$ for brevity.

We now consider various operators on $\Omega$. First of all there is the operator $d$ which has degree +1 and satisfies $d^{2}=0$. Next we have the Hochschild 
boundary operator $b$, which may be defined on homogeneous forms of degree $>0$ by

$$
b(\omega d a)=(-1)^{|\omega|}(\omega a-a \omega)
$$

where $|\omega|$ denotes the degree of $\omega$, and by zero on 0 -forms. Then $b$ has degree -1 and satisfies $b^{2}=0$.

Using $b, d$ we define the Karoubi operator $\kappa$ by

$$
b d+d b=1-\kappa .
$$

Then $\kappa$ has degree zero, and it commutes with $b$ and $d$ :

$$
[\kappa, b]=[\kappa, d]=0
$$

since $d^{2}=b^{2}=0$. It is easy to show from (2) that $\kappa$ is given in degrees $>0$ by

$$
\kappa(\omega d a)=(-1)^{|\omega|} d a \omega
$$

and by the identity in degree zero.

Finally we define Connes' operator $B$ by

$$
B=\sum_{j=0}^{n} \kappa^{j} d \quad \text { on } \Omega^{n} .
$$

Then $B$ has degree +1 and we have

$$
B d=d B=B^{2}=0 .
$$

Moreover, from (4) we have

$$
\kappa^{j}\left(d a_{0} d a_{1} \cdots d a_{n}\right)=(-1)^{n j} d a_{n-j+1} \cdots d a_{n} d a_{0} \cdots d a_{n-j}
$$

for $0 \leq j \leq n+1$, hence $\kappa^{n+1} d=d$ on $\Omega^{n}$ and

$$
\kappa B=B \kappa=B \text {. }
$$

Proposition 3.1. On elements of $\Omega^{n}$ we have the following identities:

(a) $\kappa^{n+1} d=d$.

(b) $\kappa^{n}=1+b \kappa^{n} d$.

(c) $\kappa^{n} b=b$.

(d) $\kappa^{n+1}=1-d b$.

(e) $\left(\kappa^{n}-1\right)\left(\kappa^{n+1}-1\right)=0$.

(f) $\kappa^{n(n+1)}-1=b B=-B b$.

Proof. We have already established (a).

Iterating (4) $n$ times we obtain

$$
\begin{aligned}
\kappa^{n}\left(a_{0} d a_{1} \cdots d a_{n}\right) & =d a_{1} \cdots d a_{n} a_{0} \\
& =a_{0} d a_{1} \cdots d a_{n}+\left[d a_{1} \cdots d a_{n}, a_{0}\right] \\
& =a_{0} d a_{1} \cdots d a_{n}+(-1)^{n} b\left(d a_{1} \cdots d a_{n} d a_{0}\right) \\
& =\left(1+b \kappa^{n} d\right)\left(a_{0} d a_{1} \cdots d a_{n}\right)
\end{aligned}
$$

proving (b). 
Next, by applying $b$ to both sides of (b) and using the fact that $\kappa$ and $b$ commute we obtain (c).

Applying $\kappa$ to (b) and using (a) and (3) we have

$$
\kappa^{n+1}=\kappa+b \kappa^{n+1} d=\kappa+b d=1-d b
$$

whence (d).

(e) is a consequence of (b) and (d).

Finally (f) is proved by means of geometric series:

$$
\begin{gathered}
\kappa^{n(n+1)}-1=\sum_{j=0}^{n} \kappa^{n j}\left(\kappa^{n}-1\right)=\sum_{j=0}^{n} \kappa^{n j} b \kappa^{n} d=b B \\
\kappa^{n(n+1)}-1=\sum_{j=0}^{n-1} \kappa^{(n+1) j}\left(\kappa^{n+1}-1\right)=-\sum_{j=0}^{n-1} \kappa^{(n+1) j} d b=-B b
\end{gathered}
$$

and the formulas (a)-(d).

Remarks. Since the polynomial in 3.1(e) has constant term 1, the Karoubi operator $\kappa$ is invertible on $\Omega$. It is thus a symmetry of the structure consisting of $\Omega$ and the operators $d, b, B$.

One can view $\kappa$ as an operator on the space $A \otimes \bar{A}^{\otimes n}$ of normalized chains which replaces the operator $\lambda$ given by cyclic permutation with sign on the space $A^{\otimes n+1}$ of unnormalized chains. The polynomial relation 3.1(e) is then the appropriate analogue of the fact that $\lambda$ has finite order in each degree. As we shall see, this relation gives rise to a spectral decomposition of $\Omega$ with respect to $\kappa$. The spectral projection corresponding to the eigenvalue 1 is then analogous to averaging over the cyclic group generated by $\lambda$.

Spectral decomposition with respect to $\kappa$. Let $\mathbb{C}[\kappa]$ be the algebra of polynomials in the indeterminate $\kappa$, and consider $\Omega$ as a module over $\mathbb{C}[\kappa]$, where multiplication by the indeterminate $\kappa$ is given by the operator $\kappa$. As $\Omega^{n}$ is killed by $\left(\kappa^{n}-1\right)\left(\kappa^{n+1}-1\right)$, and by $\kappa-1$ when $n=0$, we see that $\Omega$ is a torsion module over $\mathbb{C}[\kappa]$. We recall that this means we have a spectral decomposition into generalized eigenspaces

$$
\Omega=\bigoplus_{z \in \mathbb{C}} \Omega_{z}, \quad \Omega_{z}=\bigcup_{n} \operatorname{Ker}\left((\kappa-z)^{n} ; \Omega\right)
$$

where $\operatorname{Ker}(f ; \Omega)$ denotes the annihilator of $f$ on $\Omega$. The operator $\kappa-z$ is invertible on $\Omega_{z^{\prime}}$ for $z \neq z^{\prime}$.

The projection operator $P_{z}$ with image $\Omega_{z}$ given by this decomposition is the spectral projection for the operator $\kappa$ and the eigenvalue $z$. It commutes with any operator commuting with $\kappa$, and if $P_{z}^{\perp}=1-P_{z}$ is the complementary projection, then we have a splitting

$$
\Omega=P_{z} \Omega \oplus P_{z}^{\perp} \Omega
$$

where $\kappa-z$ is locally nilpotent on $P_{z} \Omega$ and invertible on $P_{z}^{\perp} \Omega$. 
So far only the fact that $\Omega$ is a torsion $\mathbb{C}[\kappa]$-module has been used, but we now examine the polynomial $\left(\kappa^{n}-1\right)\left(\kappa^{n+1}-1\right)$ for $n \geq 1$ which kills $\Omega^{n}$. The roots of this polynomial are roots of unity, and since $n$ and $n+1$ are relatively prime, this polynomial has 1 as a double root and all the other roots are simple. Consequently, if $\mu_{\infty}$ is the set of roots of unity, then we have

$$
P_{z} \Omega= \begin{cases}\operatorname{Ker}\left((\kappa-1)^{2} ; \Omega\right), & z=1, \\ \operatorname{Ker}(\kappa-z ; \Omega), & z \in \mu_{\infty}, z \neq 1, \\ 0, & z \notin \mu_{\infty},\end{cases}
$$

in degree $n$, i.e., with $\Omega$ replaced by $\Omega^{n}$. As it obviously holds in degree zero where $\kappa=1$, we then deduce (11) by taking the direct sum.

Writing $P=P_{1}$ for the spectral projection associated to the eigenvalue 1, we have the splitting

$$
\Omega=P \Omega \oplus P^{\perp} \Omega
$$

such that $(\kappa-1)^{2}=0$ on $P \Omega$ and such that $\kappa-1$ is invertible on $P^{\perp} \Omega$. In particular, on $P \Omega$ we have $\kappa^{m}=(1+(\kappa-1))^{m}=1+m(\kappa-1)$, so using 3.1(f) we find that $\kappa$ on $P \Omega$ is given by

$$
\kappa=1-\frac{1}{n(n+1)} B b \quad \text { on } P \Omega^{n}
$$

for $n \geq 1$, and by 1 for $n=0$.

This implies that $\kappa d=d$ on $P \Omega$, hence $B=(n+1) d$ on $P \Omega^{n}$ by the definition of $B$. Letting $N$ be the degree operator on $\Omega$, i.e., $N \omega=|\omega| \omega$, we thus have $B=N d$ on $P \Omega$. On the other hand $B=0$ on $P^{\perp} \Omega$, because $\kappa-1$ is invertible on this space and $B(\kappa-1)=0$. Thus we have the formula

$$
B=N P d \text {. }
$$

The spectral decomposition (9) holds for any torsion module over $\mathbb{C}[\kappa]$ in place of $\Omega$, so for any subspace $V \subset \Omega$ closed under $\kappa$ we have similar decompositions of $V$ and $\Omega / V$ which are compatible with (9). Consequently the spectral projection $P_{z}$ on $\Omega$ carries $V$ into $V$, and it induces the corresponding spectral projections for $V$ and $\Omega / V$.

We now apply this to the subspaces $F^{n} \Omega$ in the Hodge filtration $\S 1$ (6). Since $F^{n} \Omega$ is closed under $d, b$, it is closed under $\kappa$. Thus $P$ yields splittings of $F^{n} \Omega$ and $\Omega / F^{n} \Omega$ having the same properties as (12). As these splittings are compatible for different $n$, we obtain a splitting of inverse systems

$$
\theta \Omega=P \theta \Omega \oplus P^{\perp} \theta \Omega
$$

such that $(\kappa-1)^{2}=0$ on $P \theta \Omega$ and such that $1-\kappa$ is invertible on $P^{\perp} \theta \Omega$. Note that $P$ commutes with $b+B$, so (12) is a splitting of supercomplexes and (15) is a splitting of towers of supercomplexes.

Before continuing with these splittings we need some terminology. Let $K$ be a supercomplex with differential $d$. By a special contraction on $K$ we mean an odd operator $h$ such that $[d, h]=1$ and $h^{2}=0$. If such an operator exists, then $K$ is contractible, i.e., homotopy equivalent to zero. Conversely, if $K$ is 
contractible, so that there is an odd operator $h$ with $[d, h]=1$, then $h d h$ is easily seen to be a special contraction.

By a special deformation retraction on $K$ we mean a pair $(e, h)$, where $e$ and $h$ are even and odd operators respectively on $K$ satisfying

$$
\begin{gathered}
e^{2}=e, \quad[d, e]=0, \\
{[d, h]=1-e, \quad h^{2}=h e=e h=0 .}
\end{gathered}
$$

The first line means that we have a splitting $K=e K \oplus e^{\perp} K$ into subcomplexes, and the second means that $h$ is a special contraction on $e^{\perp} K$ extended to $K$ so as to be zero on $e K$. A special deformation retraction may therefore be viewed as a splitting into two subcomplexes together with a special contraction on the second subcomplex.

In this situation the inclusion $e K \rightarrow K$ and projection $e: K \rightarrow e K$ are inverse up to homotopy, so that $K$ and $e K$ are homotopy equivalent. As the subcomplex $e K$ tends to be important, we sometimes say that $(e, h)$ is a special deformation retraction of $K$ onto $e K$.

We remark that $h$ determines $e$ since $e=1-[d, h]$. One can verify that with $e$ defined this way the identities (17) are equivalent to

$$
h d h=h, \quad h^{2}=0 .
$$

Finally we note that the notions of special contraction and special deformation retraction carry over to inverse systems of supercomplexes in an obvious way.

With this terminology understood, let us return to the supercomplex $\Omega$ and the inverse systems $\left(F^{n} \Omega\right)$ and $\theta \Omega$. We then have the splitting $\Omega=P \Omega \oplus P^{\perp} \Omega$ into subcomplexes, such that $1-\kappa=[b, d]$ is invertible on $P^{\perp} \Omega$. If $G$ is the inverse, then $G$ commutes with operators commuting with $\kappa$, so we have $(G d)^{2}=0$ and

$$
[b+B, G d]=G[b+B, d]=G(1-\kappa)=1
$$

showing that $G d$ is a special contraction on $P^{\perp} \Omega$. Extending $G$ to $\Omega$ so as to vanish on $P \Omega$, we then obtain a special deformation retraction $(P, G d)$ on $\Omega$.

The same argument applies verbatim to $F^{n} \Omega$ and $\Omega / F^{n} \Omega$. Moreover, the operator $G$ on $\Omega$ induces the corresponding operator on each of these supercomplexes. Thus we have proved

Proposition 3.2. The pair $(P, G d)$ induces special deformation retractions on the supercomplex $\Omega$ and on the inverse systems of supercomplexes $\left(F^{n} \Omega\right), \theta \Omega$.

In $\S 6$ we will consider $P_{-1}$ in addition to $P=P_{1}$. Writing $P_{1,-1}=P+P_{-1}$, we then have the splitting

$$
\Omega=P \Omega \oplus P_{-1} \Omega \oplus P_{1,-1}^{\perp} \Omega
$$

such that $(\kappa-1)^{2}=0$ on $P \Omega, \kappa=-1$ on $P_{-1} \Omega$, and such that both $\kappa-1$ and $\kappa+1$ are invertible on $P_{1,-1}^{\perp} \Omega$. Furthermore we have corresponding splittings of $\left(F^{n} \Omega\right)$ and $\theta \Omega$ with the same properties. 
Remarks. (1) One has the following explicit formulas [CQ2, §2]:

$$
\begin{gathered}
G d=\frac{1}{n+1} \sum_{j=0}^{n}\left(\frac{n}{2}-j\right) \kappa^{j} d \text { on } \Omega^{n}, \\
1-P=(G d) b+b(G d) .
\end{gathered}
$$

(2) Although we have been working over the complex numbers, we would like to point out that the foregoing discussion can be carried out over any groundfield of characteristic zero with one minor change. The spectral decomposition (9) must include contributions from all monic irreducible polynomials over the groundfield, not only the linear ones. This does not affect what we have said about the spectral projections $P$ and $P_{-1}$, which is all we use in the rest of the paper. On the other hand the characteristic zero hypothesis is required in order that $\kappa^{n}-1$ have simple roots, and this is essential for (11).

\section{THE SUPERCOMPLEX $X(R)$ AND TOWER $\mathscr{X}(R, I)$}

Let $R$ be an algebra. If $M$ is an $R$-bimodule, let

$$
M_{\natural}=M /[M, R]
$$

be its commutator quotient space, and let $\natural: M \rightarrow M_{\natural}$ denote the canonical surjection. We recall that a trace on the bimodule $M$ with values in a vector space $V$ is a linear map $\tau: M \rightarrow V$ satisfying $\tau(m x)=\tau(x m)$ for $m \in M$, $x \in R$. Clearly traces $M \rightarrow V$ are equivalent to linear maps $M_{\natural} \rightarrow V$, in other words, $M_{\natural}$ is the universal target for traces on $M$.

We now consider the bottom two levels of the Hodge tower associated to $\Omega R$. As $b\left(\Omega^{n+1} R\right)=\left[\Omega^{n} R, R\right]$ by $\S 3(2)$, the ground level is

$$
\Omega R / F^{0} \Omega R=R_{\natural}
$$

where by abuse of notation we also write $R_{\natural}$ for the supercomplex given by $R_{\natural}$ in even degree and by 0 in odd degree. The next level plays an important role in this paper, so we introduce the special notation:

$$
X(R)=\Omega R / F^{1} \Omega R .
$$

It is the supercomplex

$$
R \underset{\mathrm{\natural d}}{\stackrel{\bar{b}}{\leftrightarrows}} \Omega^{1} R_{\natural}
$$

where $\bar{b}$ is defined by $\bar{b}(\lfloor(x d y))=b(x d y)=[x, y]$. The canonical surjection between these levels is the map of supercomplexes

$$
X(R) \rightarrow R_{\natural}
$$

given by $\natural: R \rightarrow R_{\natural}$. We note that there are bilinear maps

$$
(x, y) \mapsto x y \in R, \quad(x, y) \mapsto \natural(x d y) \in \Omega^{1} R_{\natural}
$$


from $R$ to the even and odd subspaces of $X(R)$, where the latter satisfies the Hochschild 1-cocycle condition:

$$
\natural(x d(y z))=\natural(x y d z)+\natural(z x d y) .
$$

The importance of $X(R)$ is due to the fact that it is a noncommutative analogue of the ordinary de Rham complex $\Omega_{R}$ for commutative algebras. To explain this we recall that $\Omega_{R}$ calculates the periodic cyclic homology when $R$ is a smooth (commutative) algebra. Now smooth algebras are defined via the lifting property with respect to nilpotent extensions in the commutative algebra category. When this lifting property is carried over to the category of all algebras, we obtain the class of quasi-free algebras studied in [CQ1]; cf. also 7.1 below for the definition. Quasi-free algebras may also be described as the algebras having Hochschild cohomological dimension $\leq 1$. Hence by the second remark following 1.1, the periodic cyclic homology for such an algebra $R$ is calculated by $X(R)$. Thus the $X$ supercomplex is analogous to the ordinary de Rham complex in the sense that it computes the periodic cyclic homology in the 'nonsingular' case.

We next consider the homology of the two levels under discussion. For the ground level the odd homology is trivial, and the even homology is the vector space

$$
R_{\mathrm{b}}=H H_{0} R=H C_{0} R
$$

which is universal for traces on $R$. By $\S 1(29)$ the homology of $X(R)$ is

$$
\begin{aligned}
& H_{+}(X(R))=H D_{0} R=\operatorname{Ker}\left\{d: R_{\natural} \rightarrow \Omega^{1} R_{\natural}\right\}, \\
& H_{-}(X(R))=H C_{1} R .
\end{aligned}
$$

We would like to give a similar dual interpretation of this homology.

We first interpret $\Omega^{1} R_{\natural}$ in terms of Hochschild 1-cocycles.

Proposition 4.1. There is a one-one correspondence $T(x d y)=f(x, y)$ between traces $T: \Omega^{1} R \rightarrow V$ and bilinear maps $f(x, y)$ from $R$ to $V$ satisfying

$$
f(x y, z)-f(x, y z)+f(z x, y)=0 .
$$

Equivalently, $\Omega^{1} R_{\natural}$ equipped with $\downarrow(x d y)$ is the universal vector space for Hochschild 1-cocycles on $R$.

Proof. It is clear that if $T$ is a trace on $\Omega^{1} R$, then $T(x d y)$ satisfies (8). Conversely, let $f$ be a bilinear map from $R$ to $V$ satisfying (8), and note that $f(x, 1)=0$ for all $x$. Since $\Omega^{1} R=R \otimes \bar{R}$, we then have a linear map $T: \Omega^{1} R \rightarrow V$ given by $T(x d y)=f(x, y)$. Applying $T$ to

$$
-[x d y, z]=x y d z-x d(y z)+z x d y
$$

we obtain $T([x d y, z])=0$, showing that $T$ is a trace.

Traces on $\Omega^{1} R$ arise in connection with homotopy for traces on $R$. Two traces $\tau_{0}, \tau_{1}$ on $R$ will be called homotopic (or cobordant [Col, II]) when $\tau_{1}-\tau_{0}=T d$ for some trace $T$ on $\Omega^{1} R$. This terminology is motivated by the following situation. 
Let $u_{t}: R \rightarrow R^{\prime}, 0 \leq t \leq 1$, be a one-parameter family of homomorphisms, and let $\tau^{\prime}$ be a trace on the algebra $R^{\prime}$. We claim that the traces $\tau^{\prime} u_{0}$ and $\tau^{\prime} u_{1}$ on $R$ are then homotopic in the above sense, provided that we can differentiate and integrate with respect to $t$ as usual. In order to avoid analytical considerations we present the argument when $u_{t}$ is a polynomial family in the sense that $u_{t} x$ is a polynomial function of $t$ with values in $R^{\prime}$ for each $x \in R$.

Proposition 4.2. The traces $\tau_{0}, \tau_{1}$ on $R$ are homotopic iff there is an algebra $R^{\prime}$, a one-parameter polynomial family of homomorphisms $u_{t}: R \rightarrow R^{\prime}$, and a trace $\tau^{\prime}$ on $R^{\prime}$ such that $\tau_{0}=\tau^{\prime} u_{0}$ and $\tau_{1}=\tau^{\prime} u_{1}$.

Proof. $(\Rightarrow)$ Suppose $T$ is a trace on $\Omega^{1} R$ such that $\tau_{1}-\tau_{0}=T d$. Let $R^{\prime}$ be the semi-direct product algebra $R \oplus \Omega^{1} R$, let $u_{t}: R \rightarrow R^{\prime}$ be the family of homomorphisms given by $u_{t} x=x+t d x$, and let $\tau^{\prime}$ be the trace on $R^{\prime}$ given by $\tau^{\prime} x=\tau_{0} x, \tau^{\prime}(x d y)=T(x d y)$. Then we have $\tau_{0}=\tau^{\prime} u_{0}, \tau_{1}=\tau^{\prime} u_{1}$.

$(\Leftrightarrow)$ Let $u_{t}: R \rightarrow R^{\prime}$ and $\tau^{\prime}$ have the indicated properties, and let $\dot{u}_{t}$ be the derivative of $u_{t}$ with respect to $t$. Then

$$
T(x d y)=\int_{0}^{1} \tau^{\prime}\left(u_{t} x \dot{u}_{t} y\right) d t
$$

defines a trace $T$ on $\Omega^{1} R$, since the right side is a Hochschild 1-cocycle. Alternatively, the integrand is the trace on $\Omega^{1} R$ obtained by pulling $\tau^{\prime}$ back by the $R$-bimodule homomorphism $\Omega^{1} R \rightarrow R^{\prime}$ induced by the derivation $\dot{u}_{t}$ relative to $u_{t}$. Finally we have $T(d y)=\tau^{\prime} u_{1}(y)-\tau^{\prime} u_{0}(y)$, hence the traces $\tau^{\prime} u_{0}, \tau^{\prime} u_{1}$ on $R$ are homotopic.

We can now proceed to the dual interpretation of $X(R)$. Consider the supercomplex $X^{\star}=\operatorname{Hom}(X(R), V)$ of linear functions on $X(R)$ with values in $V$. Even elements of this supercomplex are linear functions $g(x)$ on $R$, and odd elements can be viewed either as traces $T$ on $\Omega^{1} R$, or as Hochschild 1cocycles $f(x, y)$. The transpose of $\bar{b}$ sends $g(x)$ to $g([x, y])$, hence an even cocycle in $X^{\star}$ is the same as a trace on $R$. The transpose of $\sharp d$ is $T \mapsto T d$, or $f(x, y) \mapsto f(1, x)$, hence the even coboundaries are just the traces on $R$ which are nullhomotopic. Thus the even cohomology

$$
H^{+}\left(X^{\star}\right)=\operatorname{Hom}\left(H_{+}(X(R)), V\right)
$$

is the space of homotopy classes of traces on $R$ with values in $V$, and this implies that $H_{+}(X(R))=H D_{0} R$ is the universal space for homotopy classes of traces on $R$. Moreover, the canonical surjection $X(R) \rightarrow R_{\natural}$ can be interpreted dually as the map sending a trace to its homotopy class.

An odd cocycle in $X^{\star}$ can be viewed either as a trace $T$ on $\Omega^{1} R$ such that $T d=0$, or as a Hochschild 1-cocycle $f(x, y)$ satisfying $f(1, y)=0$. Now (8) implies $f(1, y z)=f(y, z)+f(z, y)$, so the condition $f(1, y)=0$ is equivalent to $f$ being a cyclic 1-cocycle. Furthermore, odd coboundaries are of the form $g([x, y])$, and these are the same as cyclic 1-coboundaries. Thus $H^{-}\left(X^{\star}\right)$ is the cyclic cohomology of degree one with values in $V$ in agreement with (7). 
On the other hand, a trace $T$ on $\Omega^{1} R$ such that $T d=0$ can be viewed as a homotopy from the zero trace on $R$ to itself. Thus a cyclic 1-cocycle on $R$ is analogous to a loop in the space of traces on $R$ with respect to the homotopy relation for traces defined above.

We next recall from [Q1] an important example of the supercomplex $X(R)$. Let $R$ be a free algebra, i.e., the tensor algebra $T(V)=\bigoplus_{n \geq 0} V^{\otimes n}$ on a vector space $V$. In this case we have a canonical isomorphism of $R$-bimodules

$$
R \otimes V \otimes R \stackrel{\sim}{\rightarrow} \Omega^{1} R, \quad x \otimes v \otimes y \mapsto x d v y .
$$

Passing to commutator quotient spaces yields a canonical isomorphism of vector spaces

$$
R \otimes V \stackrel{\sim}{\rightarrow} \Omega^{1} R_{\natural}, \quad x \otimes v \mapsto \natural(x d v)
$$

thereby identifying $\Omega^{1} R_{\natural}$ and $\bigoplus_{n \geq 1} V^{\otimes n}$.

Let $\sigma$ on $V^{\otimes n}$ be the forward shift cyclic permutation operator and define $N_{\sigma}$ on $V^{\otimes n}$ to be $\sum_{j=0}^{n-1} \sigma^{j}$. Then $X(R)$ is the direct sum of $\mathbb{C}$ regarded as a supercomplex concentrated in even degree and

$$
\bigoplus_{n \geq 1} V^{\otimes n} \underset{N_{\sigma}}{\stackrel{1-\sigma}{\leftrightarrows}} \bigoplus_{n \geq 1} V^{\otimes n} .
$$

Since we are in characteristic zero, the kernels of $1-\sigma$ and $N_{\sigma}$ equal the images of $N_{\sigma}$ and $1-\sigma$ respectively, so we have

$$
H_{+}(X(R))=\mathbb{C}, \quad H_{-}(X(R))=0
$$

for a free algebra.

$I$-adic filtration of $X(R)$. Let $I$ be an ideal in the algebra $R$. By the universal property of $\Omega^{1}$ with respect to derivations we have

$$
\Omega^{1}(R / I)=\Omega^{1} R / I \Omega^{1} R+\Omega^{1} R I+d I=\Omega^{1} R / I d R+d R I+d I
$$

since $\Omega^{1} R=R d R=d R R$. Passing to commutator quotient spaces yields

$$
\Omega^{1}(R / I)_{\natural}=\Omega^{1} R /\left[\Omega^{1} R, R\right]+I d R+d I=\Omega^{1} R_{\natural} / h(I d R+d I) .
$$

Consider the $I$-adic inverse system of algebras $\left(R / I^{n+1}\right)$. We then have a tower of quotient complexes of $X(R)$ given by

$$
X\left(R / I^{n+1}\right): R / I^{n+1} \leftrightarrows \Omega^{1} R_{\natural} / \hbar\left(I^{n+1} d R+d\left(I^{n+1}\right)\right)
$$

It is better, however, to work with slightly different quotient complexes having nicer properties.

We define the $I$-adic filtration of $X(R)$ to be the decreasing filtration given by the subcomplexes

$$
\begin{aligned}
& F_{I}^{2 n+1} X(R): I^{n+1} \leftrightarrows \natural\left(I^{n+1} d R+I^{n} d I\right), \\
& F_{I}^{2 n} X(R): I^{n+1}+\left[I^{n}, R\right] \leftrightarrows \natural\left(I^{n} d R\right)
\end{aligned}
$$


for $n \geq 0$ and $F_{I}^{p} X(R)=X(R)$ for $p<0$. These are closed under the differentials $\bar{b}$, $₫ d$ because $\bar{b} \natural\left(I^{n} d I\right)=\left[I^{n}, I\right] \subset I^{n+1}$ and $\natural d\left(I^{n+1}\right) \subset \sum_{0}^{n} \natural\left(I^{j} d I I^{n-j}\right)$ $\subset \mathfrak{h}\left(I^{n} d I\right)$. Since $I d R \subset d I+d I R$ we have

$$
\natural\left(I^{n+1} d R\right) \subset \emptyset\left(I^{n} d I\right)
$$

so we can shorten the formula for $F_{I}^{2 n+1} X(R)$ if we want. In practice it is usually easier to work with (15).

Corresponding to this filtration is a tower of supercomplexes $\mathscr{Z}(R, I)=$ $\left(\mathscr{X}^{p}(R, I)\right)$ where

$$
\mathscr{X}^{p}(R, I)=X(R) / F_{I}^{p} X(R)
$$

Thus we have

$$
\begin{aligned}
& \mathscr{X}^{2 n+1}(R, I): R / I^{n+1} \leftrightarrows \Omega^{1} R_{\natural} / \natural\left(I^{n+1} d R+I^{n} d I\right), \\
& \mathscr{X}^{2 n}(R, I): R / I^{n+1}+\left[I^{n}, R\right] \leftrightarrows \Omega^{1} R_{\natural} / \natural\left(I^{n} d R\right)
\end{aligned}
$$

for $n \geq 0$, and $\mathscr{X}^{p}(R, I)=0$ for $p<0$. The ground level $\mathscr{X}^{0}(R, I)$ is $(R / I)_{\natural}$ as a supercomplex concentrated in even degree. Since $\downarrow(R d I) \subset \natural(I d R+d I)$ we have

$$
\mathscr{X}^{1}(R, I)=X(R / I)
$$

and the surjection $\mathscr{X}^{1}(R, I) \rightarrow \mathscr{X}^{0}(R, I)$ can be identified with the surjection (4) for the algebra $R / I$. In particular, even when $I=0$ the tower $\mathscr{X}(R, I)$ can have two nontrivial layers. lows:

Comparing (14), (18) we have maps of quotient complexes of $X(R)$ as fol-

$$
\rightarrow X\left(R / I^{n+1}\right) \rightarrow \mathscr{X}^{2 n+1}(R, I) \rightarrow \mathscr{X}^{2 n}(R, I) \rightarrow X\left(R / I^{n}\right) \rightarrow .
$$

Thus the towers $\mathscr{X}(R, I)=\left(\mathscr{X}^{p}(R, I)\right)$ and $\left(X\left(R / I^{n}\right)\right)$ have the same inverse limit which we denote

$$
\widehat{\mathscr{X}}(R, I)=\lim _{\longleftarrow} \mathscr{X}^{p}(R, I)=\lim X\left(R / I^{n+1}\right)
$$

and call the $I$-adic completion of $X(R)$. This completion is naturally a topological supercomplex.

We next establish some functorial properties of the $I$-adic filtration $F_{I}^{p} X(R)$ and tower $\mathscr{X}(R, I)$. These are clearly functors of the pair $(R, I)$.

Lemma 4.3. One has $\mathscr{X}^{p}(R, I) \stackrel{\sim}{\rightarrow} \mathscr{X}^{p}\left(R / I^{n+1}, I / I^{n+1}\right)$ for $p \leq 2 n+1$.

Proof. In general if $\pi: R \rightarrow R^{\prime}$ is a surjective homomorphism, $I$ is an ideal in $R$, and $I^{\prime}=\pi I$, then $\pi_{*}: X(R) \rightarrow X\left(R^{\prime}\right)$ is surjective, and it maps $F_{I}^{p} X(R)$ onto $F_{I^{\prime}}^{p} X\left(R^{\prime}\right)$, so that we have the identification

$$
\mathscr{X}^{p}\left(R^{\prime}, I^{\prime}\right)=X(R) / F_{I}^{p} X(R)+\operatorname{Ker} \pi_{*} .
$$

In the case of the quotient algebra $R^{\prime}=R / I^{n+1}$ we have $\operatorname{Ker} \pi_{*} \subset F_{I}^{2 n+1} X(R, I)$ by (20), hence $\mathscr{X}^{p}\left(R^{\prime}, I^{\prime}\right)=\mathscr{X}^{p}(R, I)$ for $p \leq 2 n+1$. 
This lemma implies that the tower $\mathscr{Z}(R, I)$ can be obtained from the inverse system of algebras $\left(R / I^{n+1}\right)$. Consequently there is a map $\mathscr{X}(R, I) \rightarrow$ $\mathscr{X}(S, J)$ induced by any homomorphism $\left(R / I^{n+1}\right) \rightarrow\left(S / J^{n+1}\right)$, not only homomorphisms $(R, I) \rightarrow(S, J)$.

Given algebras $R, S$ there is a canonical surjection of supercomplexes

$$
\alpha: X(R \otimes S) \rightarrow R_{\natural} \otimes X(S)
$$

given by $\alpha(r \otimes s)=\mathfrak{h}(r) \otimes s$ and

$$
\alpha\left(\natural\left(\left(r_{1} \otimes s_{1}\right) d\left(r_{2} \otimes s_{2}\right)\right)\right)=\natural\left(r_{1} r_{2}\right) \otimes \natural\left(s_{1} d s_{2}\right) .
$$

For background we mention that $\alpha$ is induced by the canonical DG algebra surjection

$$
\Omega(R \otimes S) \rightarrow \Omega_{R}(R \otimes S)=R \otimes \Omega S
$$

from differential forms to relative differential forms with respect to $R$ (cf. [CQ1, 2.8]), and that when $R$ is the matrix algebra $M_{n} \mathbb{C}$ it is the natural trace $\operatorname{map} X\left(M_{n} S\right) \rightarrow X(S)$.

Lemma 4.4. Let $I \subset R, J \subset S$ be ideals, and let $M$ denote the ideal $I \otimes S+R \otimes J$ in $R \otimes S$. For all $p$ one has

$$
\alpha\left(F_{M}^{p} X(R \otimes S)\right) \subset \sum_{i \geq 0} \mathfrak{q}\left(I^{i}\right) \otimes F_{J}^{p-2 i} X(S) .
$$

Proof. We can suppose $p \geq 0$. Let $p=2 n$ be even. Since

$$
M^{n}=\sum_{i=0}^{n} I^{i} \otimes J^{n-i}
$$

one has

$$
\begin{aligned}
& F_{M}^{2 n} X(R \otimes S)_{+}=\sum_{i=0}^{n+1} I^{i} \otimes J^{n+1-i}+\sum_{i=0}^{n}\left[I^{i} \otimes J^{n-i}, R \otimes S\right], \\
& F_{M}^{2 n} X(R \otimes S)_{-}=\sum_{i=0}^{n} \hbar\left(\left(I^{i} \otimes J^{n-i}\right) d(R \otimes S)\right) .
\end{aligned}
$$

Using $\alpha\left(\left[r_{1} \otimes s_{2}, r_{2} \otimes s_{2}\right]\right)=\natural\left(r_{1} r_{2}\right) \otimes\left[s_{1}, s_{2}\right]$ and (23) we have

$$
\begin{aligned}
& \alpha\left(F_{M}^{2 n} X(R \otimes S)_{+}\right) \subset \sum_{i=0}^{n+1} \natural\left(I^{i}\right) \otimes J^{n-i+1}+\sum_{i=0}^{n} \natural\left(I^{i}\right) \otimes\left[J^{n-i}, S\right] \\
& \subset \sum_{i \geq 0} \mathfrak{b}\left(I^{i}\right) \otimes F_{J}^{2 n-2 i} X(S)_{+}, \\
& \alpha\left(F_{M}^{2 n} X(R \otimes S)_{-}\right) \subset \sum_{i=0}^{n} \natural\left(I^{i}\right) \otimes \natural\left(J^{n-i} d S\right) \subset \sum_{i \geq 0} \downarrow\left(I^{i}\right) \otimes F_{J}^{2 n-2 i} X(S)_{-} .
\end{aligned}
$$

This proves the lemma when $p$ is even, and the odd case is similar. 


\section{IDENTIFICATION OF $X(R A)$}

Let $R A$ be the algebra given by the space $\Omega^{+} A$ of even forms equipped with the Fedosov product

$$
\omega \circ \xi=\omega \xi-d \omega d \xi .
$$

Note that $A=\Omega^{0} A$ is a subspace of $R A$ but not a subalgebra. This subspace generates $R A$ by virtue of the identities

$$
\begin{gathered}
d a_{1} d a_{2}=a_{1} a_{2}-a_{1} \circ a_{2}, \\
a_{0} d a_{1} \cdots d a_{2 n}=a_{0} \circ\left(d a_{1} d a_{2}\right) \circ \cdots \circ\left(d a_{2 n-1} d a_{2 n}\right) .
\end{gathered}
$$

The algebra $R A$ has the following universal property [CQ1].

Proposition 5.1. Given an algebra $R$ and a linear map $\rho: A \rightarrow R$ such ihat $\rho(1)=1$, there exists a unique homomorphism $\rho_{*}: R A \rightarrow R$ such that $\rho_{*} a=\rho a$ for all $a \in A$.

Proof. The uniqueness of $\rho_{*}$ follows from (2) and (3), because one must have

$$
\rho_{*}\left(a_{0} d a_{1} \cdots d a_{2 n}\right)=\rho\left(a_{0}\right) \omega\left(a_{1}, a_{2}\right) \cdots \omega\left(a_{2 n-1}, a_{2 n}\right)
$$

where $\omega\left(a_{1}, a_{2}\right)=\rho\left(a_{1} a_{2}\right)-\rho\left(a_{1}\right) \rho\left(a_{2}\right)$ is the curvature of $\rho$.

To prove the existence, we define $\rho_{*}$ by (4) and note that it is well defined because the right side is multilinear and vanishes when $a_{i}=1$ for some $i \geq 1$. To show $\rho_{*}$ is a homomorphism, consider the subset $S \subset R A$ consisting of $x$ satisfying the condition

$$
\rho_{*}(x \circ y)=\rho_{*}(x) \rho_{*}(y) \quad \forall y \in R A .
$$

We have to show $S=R A$. Now it is easily checked that $S$ is a subalgebra of $R A$, so it suffices to verify this condition when $x$ is an element $a$ of $A$, since $A$ generates $R A$. We can suppose $y$ has the form $a_{0} w, w=d a_{1} \cdots d a_{2 n}$. Then

$$
\begin{aligned}
\rho_{*}\left(a \circ a_{0} w\right) & =\rho_{*}\left(a a_{0} w-d a d a_{0} w\right) \\
& =\rho\left(a a_{0}\right) \rho_{*}(w)-\omega\left(a, a_{0}\right) \rho_{*}(w) \\
& =\rho(a) \rho\left(a_{0}\right) \rho_{*}(w) \\
& =\rho_{*}(a) \rho_{*}\left(a_{0} w\right),
\end{aligned}
$$

concluding the proof.

Corollary 5.2. $R A$ is a free algebra.

Indeed, if we choose a lifting of $\bar{A}$ into $A$, then this lifting extends to a homomorphism $T(\bar{A}) \rightarrow R A$, which is easily seen to be an isomorphism by considering universal mapping properties.

We note that there is a canonical surjective homomorphism $R A \rightarrow A$ sending an even form to its component of degree zero. If $I A$ is the kernel, then

$$
I A=\bigoplus_{k>0} \Omega^{2 k} A
$$


and $A=R A / I A$ is a functorial way of presenting $A$ as the quotient of a free algebra. We call $A=R A / I A$ the universal extension of $A$, because by 5.1 it is universal with respect to algebra extensions $A=R / I$ equipped with a linear lifting $\rho: A \rightarrow R$ such that $\rho(1)=1$. In the same manner $R A / I A^{n+1}$ appears as the universal nilpotent extension of order $\leq n$ of $A$.

We next study $X(R A)$. As $R A$ is a free algebra, the homology of $X(R A)$ is essentially trivial as we have seen. However, this reflects the fact that $R A$ only depends on the underlying vector space of $A$ and the identity element. It is necessary to bring in the ideal $I A$ to take account of the product in $A$, and our aim is to describe $X(R A)$ in a way suitable for determining the homology of the quotient complexes $\mathscr{X}^{p}(R A, I A)$.

Consider the $R A$-bimodule $\Omega^{1}(R A)$ and its commutator quotient space. In order to avoid confusion with the differential $d$ on $\Omega A$, we use $\delta$ to denote the canonical derivation from $R A$ to $\Omega^{1}(R A)$. Since a derivation $R A \rightarrow M$ is equivalent to a lifting homomorphism from $R A$ into the semidirect product $R A \oplus M$, it follows immediately from the universal property of $R A$ that one has an equivalence between derivations $D: R A \rightarrow M$ and linear maps $A \rightarrow M$ vanishing on 1 given by restricting $D$ to $A$. Using the universal property of $\Omega^{\mathrm{i}}(R A)$ we thus obtain a canonical isomorphism of $R A$-bimodules

$$
R A \otimes \bar{A} \otimes R A \stackrel{\sim}{\rightarrow} \Omega^{1}(R A), \quad x \otimes a \otimes x^{\prime} \mapsto x \delta a x^{\prime}
$$

This gives rise to an isomorphism on commutator quotient spaces

$$
R A \otimes \bar{A} \stackrel{\sim}{\rightarrow} \Omega^{1}(R A)_{\natural}, \quad x \otimes a \mapsto \downarrow(x \delta a)
$$

which can be written

$$
\Omega^{-} A \stackrel{\sim}{\rightarrow} \Omega^{1}(R A)_{\natural}, \quad x d a \mapsto \natural(x \delta a) .
$$

From now on we identify $\Omega^{1}(R A)_{b}$ with the space of odd forms by means of this isomorphism. We then have

$$
\natural(x \delta a)=x d a
$$

for $x$ an even form and $a \in A$.

Combining this identification with $R A=\Omega^{+} A$ we can identify $X(R A)$ and $\Omega A$ as $(\mathbb{Z} / 2)$-graded vector spaces. We are now going to compute the differentials in $X(R A)$ in terms of this identification.

To avoid confusion with $b$ on $\Omega A$, let $\beta$ denote the differential in $X(R A)$ from odd degree to even: $\beta \natural(x d y)=x \circ y-y \circ x$. Then

$$
\beta(x d a)=x \circ a-a \circ x=x a-a x-d x d a+d a d x=b(x d a)-(1+\kappa) d(x d a)
$$

where $\kappa$ is the Karoubi operator $\S 3(4)$. In other words,

$$
\beta=b-(1+\kappa) d: \Omega^{-} A \rightarrow \Omega^{+} A \text {. }
$$

To obtain the other differential $\downarrow \delta$ we calculate the 1-cocycle $\downarrow(x \delta y)$ on $R A$. 
Lemma 5.3. If $y \in \Omega^{2 n} A$, then

$$
\natural(x \delta y)=-\sum_{j=0}^{n-1} \kappa^{2 j} b(x \circ y)+\sum_{j=0}^{2 n-1} \kappa^{j} d(x y)+\kappa^{2 n}(x d y) .
$$

Proof. We proceed by induction on $n$ starting from $n=0$, where the formula is simply (8). Suppose $n>0$. To prove the formula we can assume $y$ has the form $y=y^{\prime} d a_{1} d a_{2}$ with $y^{\prime} \in \Omega^{2 n-2} A$. Then

$$
\natural(x \delta y)=\natural\left(x \delta\left(y^{\prime} \circ d a_{1} d a_{2}\right)\right)=\natural\left(\left(x \circ y^{\prime}\right) \delta\left(d a_{1} d a_{2}\right)\right)+\natural\left(\left(d a_{1} d a_{2} x\right) \delta y^{\prime}\right)
$$

where we use the fact that the Fedosov product of two forms is the ordinary product when one of the forms is closed. By the induction hypothesis the second term on the right is

$$
-\sum_{j=0}^{n-2} \kappa^{2 j} b\left(\left(d a_{1} d a_{2} x\right) \circ y^{\prime}\right)+\sum_{j=0}^{2 n-3} \kappa^{j} d\left(d a_{1} d a_{2} x y^{\prime}\right)+\kappa^{2 n-2}\left(d a_{1} d a_{2} x d y^{\prime}\right) .
$$

As $\left(d a_{1} d a_{2} x\right) \circ y^{\prime}=d a_{1} d a_{2}\left(x \circ y^{\prime}\right)=\kappa^{2}\left(\left(x \circ y^{\prime}\right) d a_{1} d a_{2}\right)=\kappa^{2}(x \circ y)$ and similarly $d a_{1} d a_{2} x y^{\prime}=\kappa^{2}(x y), d a_{1} d a_{2} x d y^{\prime}=\kappa^{2}(x d y)$, this is

$$
-\sum_{j=1}^{n-1} \kappa^{2 j} b(x \circ y)+\sum_{j=2}^{2 n-1} \kappa^{j} d(x y)+\kappa^{2 n}(x d y) .
$$

Writing $x^{\prime}=x \circ y^{\prime}$ the first term is

$$
\begin{aligned}
\natural\left(x^{\prime} \delta\left(d a_{1} d a_{2}\right)\right) & =\natural\left(x^{\prime} \delta\left(a_{1} a_{2}-a_{1} \circ a_{2}\right)\right) \\
& =\natural\left\{x^{\prime} \delta\left(a_{1} a_{2}\right)-\left(x^{\prime} \circ a_{1}\right) \delta a_{2}-\left(a_{2} \circ x^{\prime}\right) \delta a_{1}\right\} \\
& =x^{\prime} d\left(a_{1} a_{2}\right)-\left(x^{\prime} \circ a_{1}\right) d a_{2}-\left(a_{2} \circ x^{\prime}\right) d a_{1} \\
& =x^{\prime} d\left(a_{1} a_{2}\right)-x^{\prime} a_{1} d a_{2}-a_{2} x^{\prime} d a_{1}+d x^{\prime} d a_{1} d a_{2}+d a_{2} d x^{\prime} d a_{1} \\
& =\left[x^{\prime} d a_{1}, a_{2}\right]+(1+\kappa)\left(d x^{\prime} d a_{1} d a_{2}\right) \\
& =-b\left(x^{\prime} d a_{1} d a_{2}\right)+(1+\kappa) d\left(x^{\prime} d a_{1} d a_{2}\right) \\
& =-b(x \circ y)+(1+\kappa) d(x y)
\end{aligned}
$$

where we have used $d(x \circ y)=d(x y)$. Adding the above expressions gives the desired formula.

Setting $x=1$ in the formula of the lemma gives

$$
\natural(\delta y)=-\left(\sum_{j=0}^{n-1} \kappa^{2 j}\right) b y+\left(\sum_{j=0}^{2 n+1} \kappa^{j}\right) d y
$$

whence the differential in $X(R A)$ from even to odd is given by

$$
\natural \delta=-N_{\kappa^{2}} b+B: \Omega^{+} A \rightarrow \Omega^{-} A
$$

where $N_{k^{2}} b$ is a suggestive notation for the operator given by $\sum_{j=0}^{n-1} \kappa^{2 j} b$ on $\Omega^{2 n} A$. 
$I A$-adic filtration. We now show that the $I A$-adic filtration $F_{I A}^{n} X(R A)$ coincides with the Hodge filtration $F^{n} \Omega A$ under our identification. To save writing we put $\Omega=\Omega A, R=R A, I=I A$. Let $I^{n}$ denote the $n$th power of $I$ in $R$, i.e., with respect to the Fedosov product.

Lemma 5.4. For $n \geq 0$, we have

$$
\begin{aligned}
I^{n} & =\bigoplus_{k \geq n} \Omega^{2 k}, \\
\natural\left(I^{n} \delta R\right) & =\bigoplus_{k \geq n} \Omega^{2 k+1}, \\
I^{n+1}+\left[I^{n}, R\right] & =b \Omega^{2 n+1} \oplus \bigoplus_{k>n} \Omega^{2 k}, \\
\natural\left(I^{n+1} \delta R+I^{n} \delta I\right) & =b \Omega^{2 n+2} \oplus \bigoplus_{k>n} \Omega^{2 k+1} .
\end{aligned}
$$

Proof. Let $J_{n}=\bigoplus_{k \geq n} \Omega^{2 k}$. From the definition of Fedosov product we have $J_{p} \circ J_{q} \subset J_{p+q}$. As $J_{1}=I$, it follows that $I^{n} \subset J_{n}$. The other inclusion $J_{n} \subset I^{n}$ follows from (3), proving the first formula.

Using this description of $I^{n}$ and (8) we have $\curvearrowleft\left(I^{n} \delta A\right)=\bigoplus_{k \geq n} \Omega^{2 k+1}$. Similarly 5.3 yields $h\left(I^{n} \delta I\right) \subset \bigoplus_{k \geq n} \Omega^{2 k+1}$. Adding these yields the second formula.

Using this formula for $\natural\left(I^{n} \delta R\right)$ we have

$$
\left[I^{n}, R\right]=\beta \natural\left(I^{n} \delta R\right)=(b-(1+\kappa) d) \bigoplus_{k \geq n} \Omega^{2 k+1} .
$$

Modulo $I^{n+1}=\bigoplus_{k>n} \Omega^{2 k}$, this is $b \Omega^{2 n+1}$, which proves the third formula.

Finally using 5.3 we see that modulo $\downarrow\left(I^{n+1} \delta R\right)=\bigoplus_{k>n} \Omega^{2 k+1}$ the space $\natural\left(I^{n} \delta I\right)$ is spanned by the elements $\natural(x \delta y)$ with $x \in \Omega^{2 n}$ and $y \in \Omega^{2}$. As $\natural(x \delta y)=-b(x y)$ plus higher order terms, we find that $\downarrow\left(I^{n} \delta I\right)$ is congruent to $b \Omega^{2 n+2}$ modulo $\bigoplus_{k>n} \Omega^{2 k+1}$, proving the last formula.

We summarize the above calculations as follows.

Theorem 5.5. There is a natural identification of $X(R A)$ with $\Omega A$ compatible with the $(\mathbb{Z} / 2)$-grading such that the canonical 1-cocycle $\downarrow(x \delta y)$ on $R A$ with values in $\Omega^{1}(R A)_{\natural}$ is given by 5.2 , and such that the differential in $X(R A)$ is given by (9) and (10). Furthermore, the filtration $F_{I A}^{n} X(R A)$ of $X(R A)$ coincides with the Hodge filtration $F^{n} \Omega A$ of $\Omega A$.

\section{6. Номотору TYPE OF $X(R A)$ AND $\mathscr{X}(R A, I A)$}

Let $X=X(R A), F^{p} X=F_{I A}^{p} X(R A), \mathscr{X}=\mathscr{X}(R A, I A)$, and $\Omega=\Omega A$. In the preceding section we identified $X$ with $\Omega$ as $(\mathbb{Z} / 2)$-graded vector spaces in such a way that the differential in $X$ is $\beta \oplus \delta$ where

$$
\begin{aligned}
& \beta=b-(1+\kappa) d: \Omega^{-} \rightarrow \Omega^{+}, \\
& \delta=-N_{\kappa^{2}} b+B: \Omega^{+} \rightarrow \Omega^{-} .
\end{aligned}
$$


Here $N_{\kappa^{2}} b$ means $\sum_{j=0}^{n-1} \kappa^{2 j} b$ on elements of $\Omega^{2 n}$ and $₫ \delta$ has been shortened to $\delta$ to simplify the notation. Furthermore, we have $F^{p} X=F^{p} \Omega$ under this identification.

Now $\beta \oplus \delta$ is similar to $b+B$ in the respect that it is a linear combination of $b$ and $d$ multiplied by polynomials in $\kappa$. In order to compare these differentials we bring in the spectral decomposition associated to the operator $\kappa$, which was discussed in $\S 3$.

Let $P$ and $P_{-1}$ be the spectral projections corresponding to the eigenvalues 1 and -1 respectively, and set $P_{1,-1}=P+P_{-1}, P_{1,-1}^{\perp}=1-P_{1,-1}$. We then have the splitting

$$
\Omega=P \Omega \oplus P_{-1} \Omega \oplus P_{1,-1}^{\perp} \Omega
$$

where $(\kappa-1)^{2}=0$ on $P \Omega, \kappa=-1$ on $P_{-1} \Omega$, and both $\kappa-1$ and $\kappa+1$ are invertible on $P_{1,-1}^{\perp} \Omega$. This splitting is respected by $d, b, B, \beta, \delta$ since these operators commute with $\kappa$. Furthermore, we have corresponding splittings with the same properties when $\Omega$ is replaced by either of the inverse systems $\left(F^{p} \Omega\right),\left(\Omega / F^{p} \Omega\right)$.

Lemma 6.1. (i) Let $c$ be the scaling operator which is multiplication by $c_{q}$ on $\Omega^{q}$, where $c_{2 n}=c_{2 n+1}=(-1)^{n} n$ !. Then on $P \Omega$ and $P_{-1} \Omega$ we have $c(\beta \oplus \delta) c^{-1}=b+B$.

(ii) On $P_{-1} \Omega$ the operator $\delta$ is an isomorphism from even degree to odd.

(iii) On $P_{1,-1}^{\perp} \Omega$ the operator $\beta$ is an isomorphism from odd degree to even. Furthermore, the corresponding assertions hold when $\Omega$ is replaced by either of the inverse systems $\left(F^{p} \Omega\right),\left(\Omega / F^{p} \Omega\right)$.

Proof. (i) We know that $\kappa b=b$ and $\kappa d=d$ on $P \Omega$ by $\S 3$ (13), and that $\kappa=-1$ on $P_{-1} \Omega$. Hence on $P \Omega$ and $P_{-1} \Omega$ we have $\kappa^{2} b=b$ and $\kappa^{2} d=d$. Thus

$$
\beta=b-(1+\kappa) d=b-\frac{1}{n+1} \sum_{j=0}^{2 n+1} \kappa^{j} d=b-\frac{1}{n+1} B
$$

on elements of degree $2 n+1$ and

$$
\delta=-\sum_{j=0}^{n-1} \kappa^{2 j} b+B=-n b+B
$$

on elements of degree $2 n$. Conjugating by the scaling operator $c$ removes these numerical constants, proving (i).

(ii) On $P_{-1} \Omega$ we have $\kappa=-1, B=0$, and $\beta$ and $\delta$ after rescaling become $b$ from odd to even and even to odd respectively. Using 3.1(a), (c) we also have $(-1)^{n} b=b$ and $(-1)^{n+1} d=d$ on elements of degree $n$, so $b=0$ on elements of odd degree and $d=0$ on elements of even degree. Since $b d+d b=1-\kappa=2$, it then follows that $b$ on $P_{-1} \Omega$ is an isomorphism from even degree to odd with inverse $\frac{1}{2} d$, proving (ii). 
(iii) On $P_{1,-1}^{\perp} \Omega$ the operator

$$
(b-(1+\kappa) d)^{2}=\kappa^{2}-1
$$

is invertible, hence so is $b=(1+\kappa) d$. Thus $\beta$, which is $b-(1+\kappa) d$ from odd degree to even, is an isomorphism.

These arguments in the case of $\Omega$ apply verbatim to $F^{p} \Omega, \Omega / F^{p} \Omega$, and the inverse systems of these spaces.

Let us now regard $\Omega$ as a supercomplex with differential $b+B$. Thus $X, \Omega$ can be viewed as supercomplexes having the same underlying ( $\mathbb{Z} / 2)$-graded vector space, but with different differentials $\beta \oplus \delta, b+B$. We can interpret (1) both as a splitting of the supercomplex $X$

$$
X=P X \oplus P_{-1} X \oplus P_{1,-1}^{\perp} X
$$

into subcomplexes and as a splitting of the supercomplex $\Omega$. Similar assertions hold for the inverse systems of supercomplexes $\left(F^{p} X\right),\left(F^{p} \Omega\right)$ and for the towers $\mathscr{X}=\left(X / F^{p} X\right), \theta \Omega=\left(\Omega / F^{p} \Omega\right)$.

By 6.1(i) we have isomorphisms

$$
c: P X \stackrel{\sim}{\rightarrow} P \Omega, \quad c: P_{-1} X \stackrel{\sim}{\rightarrow} P_{-1} \Omega
$$

compatible with differentials. Hence we have maps of supercomplexes $c P: X \rightarrow$ $\Omega, c^{-1} P: \Omega \rightarrow X$, and also with $P$ here replaced by $P_{-1}$ or $P_{1,-1}$.

Theorem 6.2. The maps

$$
c P: X \rightarrow \Omega, \quad c^{-1} P: \Omega \rightarrow X
$$

are inverse modulo homotopy, so $X$ and $\Omega$ are homotopy equivalent supercomplexes. The corresponding assertions hold when $X, \Omega$ are replaced by the inverse systems of supercomplexes $\left(F^{p} X\right),\left(F^{p} \Omega\right)$ and by the towers $\mathscr{X}, \theta \Omega$.

Proof. Since the differential $\delta$ in $P_{-1} X$ from even degree to odd is an isomorphism by 6.1(ii), the odd operator on $P_{-1} X$ which is the inverse of $\delta$ on odd elements and zero on even elements is a special contraction. Similarly by 6.1(iii) there is a special contraction on $P_{1,-1}^{\perp} X$, so on combining these we obtain a canonical special contraction on $P_{-1} X \oplus P_{1,-1}^{\perp} X$. Consequently there is a canonical special deformation retraction $(P, h)$ of $X$ onto $P X$ associated to the splitting (4). In particular $P: X \rightarrow P X$ and the inclusion $P X \subset X$ are inverses modulo homotopy.

On the other hand, we have from 3.2 a canonical special deformation retraction $(P, G d)$ of $\Omega$ onto $P \Omega$. It follows from these special deformation retractions and the first isomorphism in (5) that $c P$, which is the composition of the homotopy equivalences

$$
X \stackrel{P}{\rightarrow} P X \stackrel{c}{\rightarrow} P \Omega \subset \Omega,
$$

is a homotopy equivalence, and that $c^{-1} P$ is a homotopy inverse for $c P$.

The same arguments apply verbatim to the inverse systems $\left(F^{p} X\right), \mathscr{X}$. 
Remark. This theorem holds with $P_{1,-1}$ instead of $P$ in (6). Indeed, the map $c P_{1,-1}: X \rightarrow \Omega$ is homotopic to $c P$, because the difference factors through $P_{-1} \Omega$ which is contractible.

As a first application, we combine the result that $X$ and $\Omega$ are homotopy equivalent with the fact that $R A$ is a free algebra by 5.1 and the computation in $\S 4$ of $X(R)$ for a free algebra $R$. This yields

$$
H_{\nu}(\Omega A)=H_{\nu}(X(R A))= \begin{cases}\mathbb{C}, & \nu=+, \\ 0, & \nu=-,\end{cases}
$$

for the homology of $\Omega A$ with respect to $b+B$. Using the functoriality of this formula in the case of the homomorphism $\mathbb{C} \rightarrow A$ we deduce 1.2.

Secondly, because $F^{p} X$ and $F^{p} \Omega$ are homotopy equivalent, the homology of $F^{p} X$ is given by the computations at the end of $\S 1$. In particular we mention

$$
\bar{H} C_{n} A=H_{n-1+2 \mathbb{Z}}\left(F_{I A}^{n} X(R A)\right), \quad n \geq 0,
$$

for later reference.

Next, the result that the towers $\mathscr{X}$ and $\theta \Omega$ are homotopy equivalent implies that $\mathscr{Z}$ is a special tower whose associated homology can be expressed in terms of the cyclic type homology of $A$ as in $\S 1$ (29). Conversely, $\mathscr{X}$ may be used instead of $\theta \Omega$ to calculate the cyclic type homology and cohomology associated to $A$.

The tower $\mathscr{Z}$ is a functor of the algebra $A$, and to take account of this it is convenient to introduce the notation $\mathscr{X}_{A}=\mathscr{X}(R A, I A)$. The maps (6) determine a canonical isomorphism

$$
\mathscr{X}_{A} \simeq \theta \Omega A
$$

in the homotopy category of towers $H o \mathscr{T}$, so that $\mathscr{Z}_{A}$ represents the cyclic homology type of $A$ in the sense of the discussion at the end of $\S 2$. In particular we have the following formulas:

$$
\begin{gathered}
H C_{n} A=H_{n+2 \mathbb{Z}}\left(\mathscr{X}_{A}^{n}\right), \\
H P_{\nu} A=H_{\nu}\left(\widehat{\mathscr{X}}_{A}\right), \\
H C^{k}(A, B)=H_{k+2 \mathbb{Z}}\left(\operatorname{Hom}^{k}\left(\mathscr{X}_{A}, \mathscr{X}_{B}\right)\right) .
\end{gathered}
$$

We next discuss in more detail the meaning of (10) in the case of cyclic homology of even degree.

Let $R=R A, I=I A$. From (10) we have

$$
\begin{aligned}
H C_{2 n} A & =H_{+}\left(\mathscr{X}^{2 n}(R, I)\right) \\
& =H_{+}\left(R / I^{n+1}+\left[I^{n}, R\right] \leftrightarrows \Omega^{1} R_{\natural} / \mathfrak{\natural}\left(I^{n} d R\right)\right) \\
& =\operatorname{Ker}\left(R / I^{n+1}+[R, R] \stackrel{\delta}{\rightarrow} \Omega^{1} R_{\natural} / \mathfrak{\natural}\left(I^{n} d R\right)\right) .
\end{aligned}
$$

Thus we have an exact sequence

$$
0 \rightarrow H C_{2 n} A \stackrel{\vartheta}{\rightarrow} R / I^{n+1}+[R, R] \stackrel{\delta}{\rightarrow} \Omega^{1} R_{\natural} /\left\lfloor\left(I^{n} \delta R\right)\right.
$$

where $\gamma$ is the map on even homology induced by $c^{-1} P: \Omega / F^{2 n} \Omega \rightarrow \mathscr{Z}^{2 n}$. 
Let us now examine the result that $\gamma$ is injective more closely. On the level of dual spaces this means that any cyclic cohomology class in $H C^{2 n} A$ can be represented by a trace on $R / I^{n+1}$. We are going to prove this dual assertion by essentially the same methods used above, but in the dual setting of cochains and traces. Our purpose is to give some insight into the proof of Theorem 6.2 as well as to link our methods to the dual viewpoint of Connes and Cuntz [Co2], [CC], [Cu1], [Cu2], which played an important role in the development of the present paper.

We begin with some terminology. By a cochain on $A$ we will mean a linear functional $f$ on $\Omega$. A cochain $f$ can be identified with a sequence $\left(f_{n}\right)_{n \geq 0}$, where

$$
f_{n}\left(a_{0}, \ldots, a_{n}\right)=f\left(a_{0} d a_{1} \cdots d a_{n}\right)
$$

can be any $(n+1)$-multilinear functional on $A$ which is simplicially normalized in the sense that it vanishes whenever $a_{i}=1$ for some $i \geq 1$. The support of $f$ is the set of $n$ such that $f_{n} \neq 0$. Any operator $T$ on differential forms gives rise to a transpose operator $f \mapsto f T$ on cochains.

We next describe the traces on $R / I^{n+1}$ in cochain terms. Note that a linear functional $\tau$ on $R=\Omega^{+}$can be identified with an even cochain, i.e., with support contained in the set of even integers. Let $c$ be the scaling operator of 6.1(i), so that $\tau c^{-1}$ has the components

$$
\left(\tau c^{-1}\right)_{2 k}=\frac{(-1)^{k}}{k !} \tau_{2 k}
$$

Proposition 6.3. With this notation $\tau$ is a trace on $R$ iff $\tau c^{-1}(b+B)=0$ and $\tau c^{-1}\left(\kappa^{2}-1\right)=0$.

Proof. Since $[R, R]$ is the image of the differential $\beta$, we see that $\tau$ is a trace iff

$$
\tau \beta=\tau(b-(1+\kappa) d)=0
$$

in which case we have $0=\tau(b-(1+\kappa) d)^{2}=\tau\left(\kappa^{2}-1\right)$. But when $\tau \kappa^{2}=\tau$ the condition (17) may be rewritten $\tau c^{-1}(b+B)=0$ as in the proof of 6.1(i).

Since $I^{n+1}=\bigoplus_{k>n} \Omega^{2 k}$ by 5.4 , it is clear that $\tau\left(I^{n+1}\right)=0$ iff $\tau$ has support contained in $[0,2 n]$. Hence this proposition yields an equivalence $f=\tau c^{-1}$ between traces $\tau$ on $R / I^{n+1}$ and even $b+B$ cocycles $f$ supported in $[0,2 n]$ and fixed by $\kappa^{2}$.

Since $\gamma$ in (14) is induced by $c^{-1} P$, its transpose sends a trace $\tau$ on $R / I^{n+1}$ to the cohomology class represented by the cocycle $\tau c^{-1} P$. To see this map is surjective, let $\xi \in H C^{2 n} A$ and represent $\xi$ by an even cocycle $f$ supported in $[0,2 n]$. Now $1-P=[b+B, G d]$ as $(P, G d)$ is a special deformation retraction, so we have $f-f P=f G d(b+B)$, where $f G d$ is an odd cochain supported in $[1,2 n-1]$. But $f P(b+B)=0$ implies $f P B b=0$, whence $f P(\kappa-1)=0$ by $\S 3(13)$. Hence $\xi$ is represented by the cocycle $f P$ which 
is fixed by $\kappa$ (compare Connes' process [Co2] of replacing a cocycle by a 'normalized' cocycle in his sense). In particular, $f P$ is fixed by $\kappa^{2}$, so $\tau=f P c$ is a trace on $R / I^{n+1}$ such that $\tau c^{-1} P=f P^{2}=f P$, which proves the desired surjectivity.

Finally we mention that besides the homology groups associated to the tower $\mathscr{X}$ our methods can also be used to determine the homology of $X\left(R / I^{n+1}\right)$. This supercomplex is slightly larger than $X^{2 n+1}$ in odd degree because one divides by

$$
\natural\left(I^{n+1} \delta R+\delta\left(I^{n+1}\right)\right)=N_{\kappa^{2}} b \Omega^{n+2} \oplus \bigoplus_{k>n} \Omega^{2 k+1}
$$

instead of the last subspace described in 5.4. Only the image of $P_{1,-1}^{\perp}$ is affected by this change, and one readily calculates the homology:

$$
\begin{aligned}
& H D_{0}\left(R / I^{n+1}\right)=H_{+}\left(X\left(R / I^{n+1}\right)\right)=H D_{2 n} A, \\
& H C_{1}\left(R / I^{n+1}\right)=H_{-}\left(X\left(R / I^{n+1}\right)\right)=H C_{2 n+1} A \oplus\left(1-\kappa^{2}\right) \Omega_{\natural}^{2 n+2} .
\end{aligned}
$$

In particular any class in $H C^{2 n+1} A$ is represented by a cyclic 1-cocycle on $R / I^{n+1}$.

\section{CARTAN HOMOTOPY FORMULA}

Let $u: R \rightarrow S$ be a homomorphism of algebras. Then $u$ extends uniquely to a DG algebra homomorphism $u_{*}: \Omega R \rightarrow \Omega S$ given by

$$
u_{*}\left(x_{0} d x_{1} \cdots d x_{n}\right)=u x_{0} d\left(u x_{1}\right) \cdots d\left(u x_{n}\right) .
$$

Moreover, $u_{*}$ commutes with $b$ as well as $d$, hence it commutes with operators generated by $b, d$, e.g. $\kappa, B, P$.

Let $\dot{u}: R \rightarrow S$ be a derivation relative to $u$, i.e., $\dot{u}(x y)=\dot{u} x u y+u x \dot{u} y$. We view $\dot{u}$ as a first order variation of $u$ and define the Lie derivative $L(u, \dot{u}): \Omega R$ $\rightarrow \Omega S$ to be the induced variation of $u_{*}$ in the following sense.

Let $\mathbb{C}[\varepsilon]=\mathbb{C} \oplus \mathbb{C} \varepsilon, \varepsilon^{2}=0$ be the algebra of dual numbers. The tensor product algebra $\mathbb{C}[\varepsilon] \otimes \Omega S=\Omega S \oplus \varepsilon \Omega S$ is naturally a DG algebra such that $\varepsilon$ has degree zero and $d \varepsilon=0$. One has a homomorphism $u+\varepsilon \dot{u}$ from $R$ to the degree zero subalgebra $\mathbb{C}[\varepsilon] \otimes S$. By the universal property of $\Omega R$ this homomorphism extends uniquely to a DG algebra homomorphism $\Omega R \rightarrow \mathbb{C}[\varepsilon] \otimes \Omega S$, which we can write $f+\varepsilon g$ where $f, g: \Omega R \rightarrow \Omega S$. Clearly $f=u_{*}$, and we define $L(u, \dot{u})$ to be the map $g$. Thus $L(u, \dot{u})$ is the map such that

$$
u_{*}+\varepsilon L(u, \dot{u}): \Omega R \rightarrow \mathbb{C}[\varepsilon] \otimes \Omega S
$$

is the unique DG algebra homomorphism extending $u+\varepsilon \dot{u}$ in degree zero.

One sees easily from this definition that $L=L(u, \dot{u})$ is a derivation relative to $u_{*}$ which commutes with $d$ and restricts to $\dot{u}$ on elements of $R$. Hence one has

$$
L\left(x_{0} d x_{1} \cdots d x_{n}\right)=\dot{u} x_{0} d\left(u x_{1}\right) \cdots d\left(u x_{n}\right)+\sum_{j=1}^{n} u x_{0} d\left(u x_{1}\right) \cdots d\left(\dot{u} x_{j}\right) \cdots d\left(u x_{n}\right) .
$$


Moreover, these properties and $\S 3$ (2) imply that $L$ commutes with $b$, hence it commutes with operators generated from $b, d$ such as $\kappa, B, P$.

As a consequence the Lie derivative on differential forms descends to a map of supercomplexes $L=L(u, \dot{u}): X(R) \rightarrow X(S)$ which on elements is given by

$$
L(x)=\dot{u} x, \quad L(h(x d y))=\hbar(\dot{u} x d(u y)+u x d(\dot{u} y)) .
$$

Our aim now is to derive an analogue for the $X$ complex of the Cartan homotopy formula, namely to show that the Lie derivative $L: X(R) \rightarrow X(S)$ is homotopic to zero in an explicit way. For this purpose we need two more ingredients, the first being an appropriate interior product operation.

The derivation $\dot{u}$ extends uniquely to an $R$-bimodule map $v: \Omega^{1} R \rightarrow S$ given by $v(x d y)=u x \dot{u} y$. We define the interior product $i=i(u, \dot{u}): \Omega R \rightarrow$ $\Omega S$ to be the map of degree -1 given by the composition

$$
\Omega^{n} R=\Omega^{1} R \otimes_{R} \Omega^{n-1} R \stackrel{v \otimes u_{*}}{\longrightarrow} S \otimes_{S} \Omega^{n-1} S=\Omega^{n-1} S .
$$

One thus has the formula

$$
i\left(x_{0} d x_{1} \cdots d x_{n}\right)=u x_{0} \dot{u} x_{1} d\left(u x_{2}\right) \cdots d\left(u x_{n}\right) \text {. }
$$

From the definition given one sees that $i$ is a map of $R$-bimodules: $i(\omega x)=$ $i(\omega) u x$ and similarly for left multiplication. This implies that $i$ and $b$ anticommute:

$$
i b+b i=0 .
$$

The other ingredient needed for our homotopy formula requires that $R$ is quasi-free in the sense of [CQ1]. This means that $R$ satisfies the conditions of

Proposition 7.1. The following conditions are equivalent:

(a) $R$ has the lifting property with respect to square-zero extensions, i.e., any homomorphism $v: R \rightarrow S / J$, where $J$ is an ideal in $S$ such that $J^{2}=0$, lifts to a homomorphism $u: R \rightarrow S$. Notice that this means $R$ has the lifting property with respect to all nilpotent extensions.

(b) There exists a linear map $\phi: R \rightarrow \Omega^{2} R$ satisfying the identity

$$
\phi(x y)=x \phi y+\phi x y+d x d y \text {. }
$$

Proof. We consider the square-zero extension $R R / I R^{2}$ of $R$, which may be identified with $R \oplus \Omega^{2} R$ equipped with the Fedosov product modulo forms of degree $>2$. A map $\phi$ as in (b) is equivalent to a lifting homomorphism $l: R \rightarrow R R / I R^{2}$ via the relation $l(x)=x-\phi x$. To prove (a) implies (b), let $S=R R / I R^{2}, J=I R / I R^{2}$, and let $v$ be the obvious isomorphism of $R$ with $S / J$. The homomorphism $u$ given by (a) is then a lifting homomorphism $l$, proving (b). Conversely, given a homomorphism $v: R \rightarrow S / J, J^{2}=0$ as in (a), choose a linear lifting $\rho: R \rightarrow S$ of $v$ such that $\rho(1)=1$, and let $\rho_{*}: R R \rightarrow S$ be the induced homomorphism 5.1. Then $\rho_{*}$ lies over $v$, hence it carries $I R$ into $J$, so it kills $I R^{2}$, thereby giving a homomorphism $\tilde{v}: R R / I R^{2} \rightarrow S$ lying over $v$. Assuming (b), we have a lifting homomorphism $l: R \rightarrow R R / I R^{2}$, and then $\tilde{v} l$ is the desired homomorphism $u$ in (a). 
We can now present our Cartan homotopy formula. Let $\phi$ be as above and let $h=h^{\phi}(u, \dot{u})$ be the linear map of odd degree from $X(R)$ to $X(S)$ given by

$$
\begin{aligned}
h_{0} x & =\natural i \phi x, \\
h_{1} \natural(x d y) & =i(x d y+b(x \phi y)) .
\end{aligned}
$$

To see $h_{1}$ is well defined we verify that $x d y+b(x \phi y)$ is a Hochschild 1-cocycle and apply 4.1:

$$
\begin{aligned}
x d(y z)+b(x \phi(y z)) & =x y d z+x d y z+b(x y \phi z+x \phi y z+x d y d z) \\
& =x y d z+x d y z+b(x y \phi z)+b(z x \phi y)-[x d y, z] \\
& =(x y d z+b(x y \phi z))+(z x d y+b(z x \phi y)) .
\end{aligned}
$$

Here we use that $b$ kills $[x \phi y, z]$, which follows from $b^{2}=0$.

Let $\partial$ stand for the differential $(\bar{b}, \emptyset d)$ in the $X$ complex.

Proposition 7.2. One has $L=\partial h+h \partial$.

Proof. We have

$$
\left(\bar{b} h_{0}+h_{1} \natural d\right) x=b i \phi x+i(d x+b \phi x)=i(d x)=\dot{u} x=L(x)
$$

establishing the formula in even degree.

Next we have

$$
\left(\natural d h_{1}\right) \natural(x d y)=\natural d i(x d y+b(x \phi y))=\natural d(u x \dot{u} y)=\natural(d(u x) \dot{u} y+u x d(\dot{u} y))
$$

where $\sharp d i b=-\sharp d b i=0$, since $\sharp d b=0$ on $\Omega^{1} S$. Also we have

$$
\begin{aligned}
\left(h_{0} \bar{b}\right) \natural(x d y) & =h_{0} b(x d y) \\
& =\natural i \phi(x y-y x) \\
& =\natural i\{[x, \phi y]+[\phi x, y]+d x d y-d y d x\} \\
& =\natural\{[u x, i \phi y]+[i \phi x, u y]+\dot{u} x d(u y)-\dot{u} y d(u x)\} \\
& =\natural(\dot{u} x d(u y)-\dot{u} y d(u x))
\end{aligned}
$$

as $i$ is a bimodule map over $R$. Combining these gives

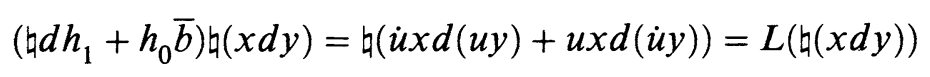

completing the proof.

We record for later reference the formula

$$
h_{0} b(x d y)=\mathfrak{t}(\dot{u} x d(u y)-\dot{u} y d(u x))
$$

established in the course of this proof.

Adic behavior. Let $I \subset R, J \subset S$ be ideals and consider the corresponding adic filtrations of the $X$ complexes $\S 4$ (15). We recall that if $u(I) \subset J$, then $u_{*}$ maps $F_{I}^{p} X(R)$ into $F_{J}^{p} X(S)$ for all $p$. We next examine the adic behavior of the Lie derivative $L=L(u, \dot{u})$. 
Proposition 7.3. Assume $u(I) \subset J$. Then for all $p$ one has

$$
L\left(F_{I}^{p} X(R)\right) \subset\left\{\begin{array}{l}
F_{J}^{p-2} X(S), \\
F_{J}^{p} X(S) \text { if } \dot{u}(I) \subset J .
\end{array}\right.
$$

Proof. One may verify this directly from the definition of the adic filtration using the derivation property of the Lie derivative, but a less computational proof with other uses goes as follows.

Let us return to the definition of the Lie derivative on differential forms as the coefficient of $\varepsilon$ in the DG algebra homomorphism $\Omega R \rightarrow \mathbb{C}[\varepsilon] \otimes \Omega S$ extending $u+\varepsilon \dot{u}: R \rightarrow \mathbb{C}[\varepsilon] \otimes S$. We note that this DG algebra homomorphism is the composition

$$
\Omega R \stackrel{(u+\varepsilon \dot{u})}{\longrightarrow} \Omega(\mathbb{C}[\varepsilon] \otimes S) \stackrel{\tilde{\alpha}}{\longrightarrow} \mathbb{C}[\varepsilon] \otimes \Omega S
$$

where $\tilde{\alpha}$ is the canonical surjection extending the identity in degree zero. It follows that $L: X(R) \rightarrow X(S)$ is the coefficient of $\varepsilon$ in the composition

$$
X(R) \stackrel{(u+\varepsilon \dot{u}) *}{\longrightarrow} X(\mathbb{C}[\varepsilon] \otimes S) \stackrel{\tilde{\alpha}}{\longrightarrow} \mathbb{C}[\varepsilon] \otimes X(S)
$$

where $\alpha$ is induced by $\tilde{\alpha}$. We note also that $\alpha$ is a case of the map $\alpha$ in 4.4.

Now $u+\varepsilon \dot{u}$ carries $I$ into the ideal $M=K \otimes S+\mathbb{C}[\varepsilon] \otimes J$ of $\mathbb{C}[\varepsilon] \otimes S$, where $K=\mathbb{C} \varepsilon$ in general and $K=0$ in case $\dot{u}(I) \subset J$. Hence $(u+\varepsilon \dot{u})_{*}$ carries $F_{I}^{p} X(R)$ into $F_{M}^{p} X(\mathbb{C}[\varepsilon] \otimes S)$, which by 4.4 is carried by $\alpha$ into

$$
\sum_{i \geq 0} \natural\left(K^{i}\right) \otimes F_{J}^{p-2 i} X(S) \subset \begin{cases}F_{J}^{p} X(S)+\varepsilon F_{J}^{p-2} X(S), & K=\mathbb{C} \varepsilon, \\ F_{J}^{p} X(S)+\varepsilon F_{J}^{p} X(S), & K=0,\end{cases}
$$

whence the result.

The next proposition describes the adic behavior of the Cartan homotopy $h=h^{\phi}(u, \dot{u})$.

Proposition 7.4. Assume $u(I) \subset J$. Then for all $p$

$$
h\left(F_{I}^{p} X(R)\right) \subset \begin{cases}F_{J}^{p-2} X(S), & \\ F_{J}^{p-1} X(S), & \dot{u}(I) \subset J, \\ F_{J}^{p} X(S), & \dot{u}(R) \subset J .\end{cases}
$$

Proof. Let $n \geq 0$. By induction we obtain the following iterated version of (1):

$$
\begin{aligned}
\phi\left(x_{0} \cdots x_{n}\right)= & \sum_{0 \leq j \leq n} x_{0} \cdots x_{j-1} \phi\left(x_{j}\right) x_{j+1} \cdots x_{n} \\
& +\sum_{0 \leq j<k \leq n} x_{0} \cdots x_{j-1} d x_{j} x_{j+1} \cdots x_{k-1} d x_{k} x_{k+1} \cdots x_{n} .
\end{aligned}
$$


Since $h_{0}=\natural i \phi$ and $i \phi(R) \subset i(R d R d R) \subset S \dot{u}(R) d S$, we have

$$
\begin{aligned}
h_{0}\left(I^{n+1}\right) & \subset \emptyset i\left(\sum_{0 \leq j \leq n} I^{j} \phi(I) I^{n-j}+\sum_{0 \leq j<k \leq n} I^{j} d I I^{k-1-j} d I I^{n-k}\right) \\
& \subset \emptyset\left(\sum_{0 \leq j \leq n} J^{j} \dot{u}(R) d S J^{n-j}+\sum_{0 \leq j<k \leq n} J^{j} \dot{u}(I) J^{k-1-j} d J J^{n-k}\right) \\
& \subset \emptyset\left(J^{n} \dot{u}(R) d S+\sum_{0 \leq i<n} J^{n-1-i} \dot{u}(I) J^{i} d J\right) .
\end{aligned}
$$

Thus

$$
h_{0}\left(I^{n+1}\right) \subset \begin{cases}\natural\left(J^{n} d S+J^{n-1} d J\right), & \\ \natural\left(J^{n} d S\right), & \dot{u}(I) \subset J, \\ \natural\left(J^{n+1} d S+J^{n} d J\right), & \dot{u}(R) \subset J .\end{cases}
$$

Using (3) we have

$$
\begin{aligned}
h_{0}\left(\left[I^{n}, R\right]\right) & \subset h_{0} b\left(I^{n} d R\right) \subset \emptyset\left(\dot{u}\left(I^{n}\right) d S+\dot{u}(R) d\left(J^{n}\right)\right) \\
& \subset \emptyset\left(\sum_{0 \leq j<n} J^{n-1-j} \dot{u}(I) J^{j} d S+\sum_{0 \leq j<n} J^{n-1-j} \dot{u}(R) J^{j} d J\right) .
\end{aligned}
$$

Thus

$$
h_{0}\left(I^{n+1}+\left[I^{n}, R\right]\right) \subset \begin{cases}\natural\left(J^{n-1} d S\right), & \\ \natural\left(J^{n} d S+J^{n-1} d J\right), & \dot{u}(I) \subset J, \\ \natural\left(J^{n} d S\right), & \dot{u}(R) \subset J .\end{cases}
$$

Since $b\left(I^{n} \phi R\right) \subset b\left(I^{n} d R d R\right) \subset\left[I^{n} d R, R\right]$, one has

$$
h_{1} \curvearrowleft\left(I^{n} d R\right) \subset i\left(I^{n} d R+b\left(I^{n} \phi R\right)\right) \subset i\left(I^{n} d R\right) \subset J^{n} \dot{u}(R) .
$$

Thus

$$
h_{1} \downarrow\left(I^{n} d R\right) \subset \begin{cases}J^{n}+\left[J^{n-1}, S\right], & \\ J^{n}, & \dot{u}(I) \subset J, \\ J^{n+1}+\left[J^{n}, S\right], & \dot{u}(R) \subset J .\end{cases}
$$

Finally

$$
h_{1} \curvearrowleft\left(I^{n} d I\right) \subset i\left(I^{n} d I+b\left(I^{n} \phi I\right)\right) \subset i\left(I^{n} d I+\left[I^{n} d R, R\right]\right) \subset J^{n} \dot{u}(I)+\left[J^{n} \dot{u}(R), S\right]
$$

so

$$
h_{1} \curvearrowleft\left(I^{n+1} d R+I^{n} d I\right) \subset \begin{cases}J^{n}, \\ J^{n+1}+\left[J^{n}, S\right], & \dot{u}(I) \subset J, \\ J^{n+1}, & \dot{u}(R) \subset J .\end{cases}
$$

The proposition follows from (5)-(8). 
Finally we would like to point out that by means of the argument used to prove 7.3 one can deduce the case of the preceding proposition in which there is no restriction on $\dot{u}$ from the case $\dot{u}(I) \subset J$. We first give the argument in a general situation.

Proposition 7.5. Let $T=\bigoplus_{n \geq 0} T_{n}$ be a graded algebra, and let $v: R \rightarrow T \otimes S$ be a homomorphism. Write $v=\sum v_{n}$, where $v_{n}: R \rightarrow T_{n} \otimes S$, and let $D v$ be the derivation $\sum n v_{n}$ relative to $v$. Suppose $I \subset R, J \subset S$ are ideals such that $v_{0}(I) \subset T_{0} \otimes J$. Then both maps $\alpha L(v, D v), \alpha h^{\phi}(v, D v): X(R) \rightarrow T_{\natural} \otimes X(S)$ carry $F_{I}^{p} X(R)$ into $\bigoplus_{n \geq 0}\left(T_{\natural}\right)_{n} \otimes F_{J}^{p-2 n} X(S)$ for all $p$.

Proof. Let $M$ be the ideal $T_{\geq 1} \otimes S+T \otimes J$ of $T \otimes S$, where $T_{\geq 1}=T_{1} \oplus T_{2} \oplus \cdots$. Then $v(I) \subset M, D v(R) \subset M$, so by the last cases of 7.3 and 7.4 we know that $L(v, D v), h^{\phi}(v, D v)$ send $F_{I}^{p} X(R)$ into $F_{M}^{p} X(T \otimes S)$. This in turn by 4.4 is mapped by $\alpha$ into

$$
\begin{aligned}
\bigoplus_{i \geq 0} \natural\left(\left(T_{\geq 1}\right)^{i}\right) \otimes F_{J}^{p-2 n} X(S) \subset & \left(T_{0} \oplus T_{1} \oplus \cdots\right) \otimes F_{J}^{p} X(S) \\
& +\natural\left(T_{1} \oplus \cdots\right) \otimes F_{J}^{p-2} X(S)+\cdots
\end{aligned}
$$

whence the result.

We apply this when $T=\mathbb{C}[\varepsilon]$ with $\varepsilon$ of degree one and $v=u+\varepsilon \dot{u}: R \rightarrow T \otimes$ $S$. In this case $D v=\varepsilon \dot{u}$ and one has $\alpha L(v, D v)=\varepsilon L(u, \dot{u}), \alpha h^{\phi}(v, D v)=$ $\varepsilon h^{\phi}(u, \dot{u})$, and this proposition yields the first cases of 7.3 and 7.4.

\section{HOMOTOPY PROPERTIES}

The Cartan homotopy formula concerns an infinitesimal change in a homomorphism. We now integrate this formula in the case of a suitable oneparameter family of homomorphisms $u_{t}: R \rightarrow S$ in order to show that the maps $u_{t^{*}}: X(R) \rightarrow X(S)$ for different $t$ are homotopic. In keeping with our algebraic setting we restrict to families depending on $t$ in a polynomial manner, so that we can differentiate and integrate with the usual rules. By introducing topologies and suitable differentiability hypotheses one can handle more general families by means of the same formulas.

We begin with some preliminaries on polynomial families.

Let $W$ be a vector space, and let $W[t]=W \otimes \mathbb{C}[t]$ be the space of polynomials in the indeterminate $t$ with coefficients in $W$. We may interpret $t$ as a complex variable and identify elements of $W[t]$ with polynomial functions from $\mathbb{C}$ to $W$.

A family of maps $f_{t}: V \rightarrow W, t \in \mathbb{C}$, will be called a polynomial family when $f_{t} v$ is a polynomial in $t$ for all $v \in V$. Such a family is equivalent to a map $V \rightarrow W[t]$.

Since the operations of differentiation and integration on polynomials are given algebraically by suitably rescaling the coefficients, it is clear that these operations make sense for polynomial families, and moreover they satisfy the 
usual properties such as

$$
f_{1}-f_{0}=\int_{0}^{1}\left(\frac{d}{d t} f_{t}\right) d t
$$

Suppose given a polynomial family of homomorphisms $u_{t}: R \rightarrow S, t \in \mathbb{C}$, i.e., a homomorphism $R \rightarrow S[t]$. The derivative

$$
\dot{u}_{t}=\frac{d}{d t} u_{t}
$$

is defined, and it is a polynomial family such that $\dot{u}_{t}$ is a derivation relative to $u_{t}$. Moreover the maps $u_{t *}, L\left(u_{t}, \dot{u}_{t}\right)$ from $X(R)$ to $X(S)$ form polynomial families such that

$$
\frac{d}{d t} u_{t *}=L\left(u_{t}, \dot{u}_{t}\right)
$$

Assuming $\phi$ given for $R$ as in 7.1 (hence $R$ must be quasi-free), we have $l\left(u_{t}, \dot{u}_{t}\right)=\left[\partial, h^{\phi}\left(u_{t}, \dot{u}_{t}\right)\right]$ by 7.2 . Now $h^{\phi}\left(u_{t}, \dot{u}_{t}\right)$ is easily seen to be a polynomial family, so the integral

$$
H^{\phi}\left(u_{t}\right)=\int_{0}^{1} h^{\phi}\left(u_{t}, \dot{u}_{t}\right) d t
$$

is a well-defined map of odd degree from $X(R)$ to $X(S)$. We have

$$
\left[\partial, H^{\phi}\left(u_{t}\right)\right]=\int_{0}^{1}\left[\partial, h^{\phi}\left(u_{t}, \dot{u}_{t}\right)\right] d t=\int_{0}^{1}\left(\frac{d}{d t} u_{t *}\right) d t=u_{1 *}-u_{0 *}
$$

which proves the following homotopy property for the $X$ complex.

Proposition 8.1. The map $H^{\phi}\left(u_{t}\right): X(R) \rightarrow X(S)$ is a (chain) homotopy between the induced maps $u_{t *}: X(R) \rightarrow X(S)$ for $t=0,1$. Consequently $u_{t *}$ is independent of $t$ modulo homotopy.

We next consider the situation where ideals are present. Associated to a pair $(R, I)$ there is the inverse system $\left(F_{I}^{p} X(R)\right)$ of subcomplexes of $X(R)$, the corresponding tower $\mathscr{X}(R, I)$ of quotient complexes, and the completion $\widehat{\mathscr{X}}(R, I)$. These objects are functorial with respect to homomorphisms $u:(R, I) \rightarrow(S, J)$.

Assume $\phi$ given for $R$ and consider a polynomial family of homomorphisms $u_{t}:(R, I) \rightarrow(S, J)$, or equivalently a homomorphism $(R, I) \rightarrow(S[t], J[t])$. Define $\varepsilon$ to be the number 0 in the restricted case where $u_{t}$ is constant modulo $J$, and put $\varepsilon=1$ in general. By 7.3 we have for each $p$ a polynomial family of maps

$$
h^{\phi}\left(u_{t}, \dot{u}_{t}\right): F_{I}^{p} X(R) \rightarrow F_{J}^{p-\varepsilon} X(S),
$$

hence we obtain

Lemma 8.2. The map $H^{\phi}\left(u_{t}\right): X(R) \rightarrow X(S)$ carries $F_{I}^{p} X(R)$ into $F_{J}^{p-\varepsilon} X(S)$ for all $p$.

Combining this with 6.2 we derive the following homotopy property for $H D_{n}$ and $H P_{\nu}$; cf. [G]. Recall that $H D_{n}=S H C_{n+2} \subset H C_{n}$. 
Proposition 8.3. If $v_{t}: A \rightarrow B$ is a polynomial family of homomorphisms, then the induced maps $v_{t *}: H D_{n} A \rightarrow H D_{n} B$ and $v_{t *}: H P_{\nu} A \rightarrow H P_{\nu} B$ are independent of $t$.

Proof. The family of homomorphisms $u_{t}=R v_{t}:(R A, I A) \rightarrow(R B, I B)$ induced by $v_{t}$ is easily seen to be a polynomial family. Using 8.2 we see that $H^{\phi}\left(u_{t}\right)$ induces a map $\mathscr{X}^{n+2}(R A, I A) \rightarrow \mathscr{X}^{n}(R B, I B)$, which is a homotopy between the maps

$$
\mathscr{X}^{n+2}(R A, I A) \rightarrow \mathscr{X}^{n}(R A, I A) \stackrel{u_{t *}}{\longrightarrow} \mathscr{X}^{n}(R B, I B)
$$

for $t=0,1$. It follows by passing to homology of degree $n+2 \mathbb{Z}$ that the maps

$$
H C_{n+2} A \stackrel{S}{\rightarrow} H C_{n} A \stackrel{v_{t *}}{\rightarrow} H C_{n} B
$$

coincide for $t=0,1$.

We also have a map of completions $\widehat{\mathscr{X}}(R A, I A) \rightarrow \widehat{\mathscr{X}}(R B, I B)$ induced by $H^{\phi}\left(u_{t}\right)$, which is a homotopy between the maps between these completions induced by $u_{0}, u_{1}$. Consequently $v_{0}, v_{1}$ induce the same map on periodic cyclic homology.

Let us return to the homomorphism $u_{t}:(R, I) \rightarrow(S[t], J[t])$ and consider the inverse system $\left(F_{I}^{p} X(R)\right)$ of supercomplexes and similarly for $(S, J)$. The following is clear from 8.2.

Proposition 8.4. The map $H^{\phi}\left(u_{t}\right): X(R) \rightarrow X(S)$ induces a map of inverse systems $H^{\phi}\left(u_{t}\right):\left(F_{I}^{p} X(R)\right) \rightarrow\left(F_{J}^{p-\varepsilon} X(S)\right)$ which is a homotopy between the induced maps $u_{t *}:\left(F_{I}^{p} X(R)\right) \rightarrow\left(F_{J}^{p-\varepsilon} X(S)\right)$ for $t=0,1$.

A similar result holds for the tower $\mathscr{X}(R, I)$, but one can do better. The following is immediate from 4.3.

Lemma 8.5. Let $R_{n}=R / I^{n+1}, I_{n}=I / I^{n+1}$. Then the canonical surjections $R \rightarrow R_{n}$ induce an isomorphism of inverse systems

$$
\mathscr{X}(R, I) \stackrel{\sim}{\rightarrow} \underset{\lim }{\longleftarrow} \mathscr{X}\left(R_{n}, I_{n}\right),
$$

i.e., a corresponding isomorphism at each level.

As $I_{n}$ is the kernel of the surjection $R_{n} \rightarrow R_{0}$, this shows that the tower $\mathscr{X}(R, I)$ can be obtained from the tower of algebras $\left(R_{n}\right)$. Consequently, if $(S, J)$ is another pair and $S_{n}=S / J^{n+1}$, then we have an induced map $\mathscr{Z}(R, I) \rightarrow \mathscr{Z}(S, J)$ associated not only to a homomorphism $(R, I) \rightarrow(S, J)$ but to any homomorphism $\left(R_{n}\right) \rightarrow\left(S_{n}\right)$.

It will be useful to give some equivalent descriptions of homomorphisms $\left(R_{n}\right) \rightarrow\left(S_{n}\right)$. Let $\widehat{R}=\lim _{n}, \widehat{I}=R_{n} I_{n}$ be the $I$-adic completions of $R, I$, and define $\widehat{S}, \widehat{J}$ similarly. These completions have natural topologies, e.g., a neighborhood basis of zero in $\widehat{R}$ is given by the completions $\widehat{I^{m}}, m \geq 0$. The following is easily verified. 
Proposition 8.6. The following data are equivalent:

(i) A homomorphism $u:\left(R_{n}\right) \rightarrow\left(S_{n}\right)$ of towers of algebras.

(ii) A compatible family of homomorphisms $u^{n}: R \rightarrow S_{n}$ such that $u^{0}(I)=0$. Note that in this situation $u^{n}$ is a homomorphism $(R, I) \rightarrow\left(S_{n}, J_{n}\right)$.

(iii) $A$ homomorphism $(R, I) \rightarrow(\widehat{S}, \widehat{J})$.

(iv) A continuous homomorphism $(\widehat{R}, \widehat{I}) \rightarrow(\widehat{S}, \widehat{J})$.

The next proposition gives the functorial and homotopy behavior of $\mathscr{X}(R, I)$ with respect to $\left(R_{n}\right)$.

Proposition 8.7. (a) A homomorphism $u:\left(R_{n}\right) \rightarrow\left(S_{n}\right)$ induces a map $u_{*}$ : $\mathscr{X}(R, I) \rightarrow \mathscr{X}(S, J)$ of towers of supercomplexes, and in this way $\mathscr{X}(R, I)$ becomes a functor of $\left(R_{n}\right)$.

(b) Assuming $\phi$ given for $R$, a homomorphism $u_{t}:\left(R_{n}\right) \rightarrow\left(S_{n}[t]\right)$ determines a map $H^{\phi}\left(u_{t}\right):\left(\mathscr{X}^{p}(R, I)\right) \rightarrow\left(\mathscr{X}^{p-\varepsilon}(S, J)\right)$ which is a homotopy between the induced maps $u_{t *}:\left(\mathscr{X}^{p}(R, I)\right) \rightarrow\left(\mathscr{X}^{p-\varepsilon}(S, J)\right)$ for $t=0,1$.

Proof. We have already established (a) by observing that $u$ gives compatible homomorphisms $\left(R_{n}, I_{n}\right) \rightarrow\left(S_{n}, J_{n}\right)$, which give compatible maps $\mathscr{X}\left(R_{n}, I_{n}\right) \rightarrow$ $\mathscr{X}\left(S_{n}, J_{n}\right)$, which in turn yield the desired map $u_{*}: \mathscr{X}(R, I) \rightarrow \mathscr{X}(S, J)$ by 8.5. However, for the homotopy assertion (b) we want a different construction of $u_{*}$ because $R_{n}$ need not be quasi-free when $R$ is quasi-free. Instead we observe that $u$ is equivalent by 8.6 to a compatible family of homomorphisms $u^{n}:(R, I) \rightarrow\left(S_{n}, J_{n}\right)$, so we have compatible maps $u_{*}^{n}: \mathscr{X}(R, I) \rightarrow \mathscr{X}\left(S_{n}, J_{n}\right)$ which yield $u_{*}$ by 8.5 . Similarly if $u_{t}^{n}:(R, I) \rightarrow\left(S_{n}[t], J_{n}[t]\right)$ is the compatible family of homomorphisms corresponding to $u_{t}$, then we have compatible maps of towers

$$
H^{\phi}\left(u_{t}^{n}\right):\left(\mathscr{X}^{p}(R, I)\right) \rightarrow\left(\mathscr{X}^{p-\varepsilon}\left(S_{n}, J_{n}\right)\right)
$$

so using 8.5 we obtain a map

$$
H^{\phi}\left(u_{t}\right):\left(\mathscr{X}^{p}(R, I)\right) \rightarrow\left(\mathscr{Z}^{p-\varepsilon}(S, J)\right)
$$

which is easily seen to have the properties asserted in (b).

Finally we consider the completion

$$
\widehat{\mathscr{X}}(R, I)=\lim _{\longleftrightarrow} \mathscr{X}^{p}(R, I)=\lim X\left(R / I^{n}\right) .
$$

In the first place this is naturally a topological supercomplex with the inverse limit topology. Secondly, if $I, J$ are two ideals defining the same adic topology, i.e., $I \subset J^{m}, J \subset I^{m}$ for some $m$, then the inverse systems $\left(R / I^{n}\right),\left(R / J^{n}\right)$ are isomorphic as pro-objects and hence we have a canonical isomorphism

$$
\widehat{\mathscr{X}}(R, I)=\widehat{\mathscr{X}}(R, J) \text {. }
$$

The following proposition describes the functorial and homotopy properties of the completion.

Proposition 8.8. (a) A continuous homomorphism $u: \widehat{R} \rightarrow \widehat{S}$ induces a continuous map $u_{*}: \widehat{\mathscr{X}}(R, I) \rightarrow \widehat{\mathscr{X}}(S, J)$ of topological supercomplexes. 
(b) Assuming $\phi$ given for $R$, a continuous homomorphism $u_{t}: \widehat{R} \rightarrow S[t]^{\wedge}$ determines a continuous map $H^{\phi}\left(u_{t}\right): \widehat{\mathscr{X}}(R, I) \rightarrow \widehat{\mathscr{X}}(S, J)$ of odd degree which is a homotopy between the induced maps $u_{0 *}, u_{1 *}$.

Proof. (a) If $u: \widehat{R} \rightarrow \widehat{S}$ is a continuous homomorphism, then $u^{-1} \widehat{I}$ is open, hence it contains $\widehat{I^{m}}$ for some $m$. Replacing $I$ by $I^{m}$ does not change the completions, so we can suppose $u$ is a continuous homomorphism $(\widehat{R}, \widehat{I}) \rightarrow$ $(\widehat{S}, \widehat{J})$. In this case $u$ is equivalent by 8.6 to a homomorphism $\left(R_{n}\right) \rightarrow\left(S_{n}\right)$, and we have an induced map of towers $\mathscr{X}(R, I) \rightarrow \mathscr{X}(S, J)$, hence a continuous map on completions by taking the inverse limit.

(b) Here $S[t]^{\wedge}=\lim S_{n}[t]$ is the algebra of formal power series in $t$ whose coefficients form a sequence in $\widehat{S}$ tending to zero. As in the proof of (a), we can suppose after replacing $I$ by some power that $u_{t}$ corresponds to a homomorphism $\left(R_{n}\right) \rightarrow\left(S_{n}[t]\right)$. The desired continuous map $H^{\phi}\left(u_{t}\right)$ is then obtained from the corresponding map in $8.7(\mathrm{~b})$ by taking the induced map on inverse limits.

\section{DeRIVED FUNCTOR ANALOGY}

In this section we apply our homotopy results to develop an analogy with the construction of derived functors, in which an extension $A=R / I$ with $R$ quasi-free plays the role of a projective resolution of $A$, and the tower $\mathscr{X}(R, I)$ corresponds to a functor applied to this resolution. In this way we establish that up to homotopy equivalence this tower is independent of the choice of quasifree extension.

Consider an algebra $R$ and an algebra extension $B=S / J$. If $R$ is a free algebra, then any homomorphism $v: R \rightarrow B$ can be lifted to a homomorphism $u: R \rightarrow S$. The same is true by 7.1 if $R$ is quasi-free and $S$ is a nilpotent extension of $B$.

Lemma 9.1. Assume either that $R$ is free or that $R$ is quasi-free and $S$ is a nilpotent extension of $B$. Given homomorphisms $v_{t}: R \rightarrow B[t]$ and $u_{0}, u_{1}: R \rightarrow S$ lifting $v_{0}, v_{1}$, there exists a homomorphism $u_{t}: R \rightarrow S[t]$ which lifts $v_{t}$ and joins $u_{0}, u_{1}$.

We consider the diagram with exact rows

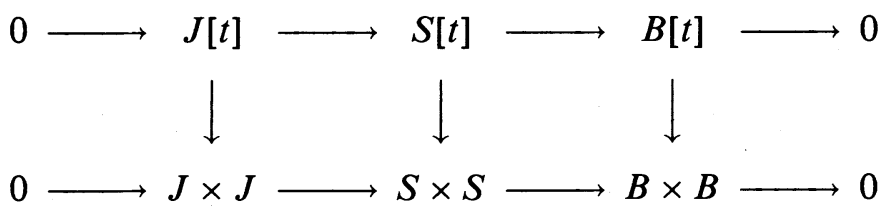

where the vertical arrows evaluate a polynomial at 0,1 . These arrows are surjective, hence we obtain a surjective algebra homomorphism

$$
S[t] \rightarrow(S \times S) \times_{(B \times B)} B[t]
$$

whose kernel is contained in $J[t]$. Now the homomorphism $u_{t}: R \rightarrow S[t]$ we seek is a lifting of the homomorphism $\left(\left(u_{0}, u_{1}\right), v_{t}\right)$ from $R$ to this fibre 
product algebra. This lifting exists when $R$ is free by the surjectivity. When $J$ is nilpotent, so is $J[t]$, hence $S[t]$ is a nilpotent extension of the fibre product, and the lifting exists if $R$ is quasi-free.

We next establish similar lifting properties for the towers of algebras $\left(R_{n}\right)$, $\left(S_{n}\right)$ associated to pairs $(R, I),(S, J)$.

Proposition 9.2. Assume $R$ is quasi-free.

(i) Given a homomorphism $v: R_{0} \rightarrow S_{0}$, there is a homomorphism $u:\left(R_{n}\right) \rightarrow$ $\left(S_{n}\right)$ which lifts $v$, i.e., is such that $u$ at level zero is equal to $v$.

(ii) Given a homomorphism $v_{t}: R_{0} \rightarrow S_{0}[t]$ and liftings $u_{0}, u_{1}:\left(R_{n}\right) \rightarrow\left(S_{n}\right)$ of $v_{0}, v_{1}$ respectively, there is a homomorphism $u_{t}:\left(R_{n}\right) \rightarrow\left(S_{n}[t]\right)$ which lifts $u_{t}$ and joins $u_{0}, u_{1}$.

Proof. (i) The desired homomorphism $u$ is equivalent to a compatible family of homomorphisms $u^{n}: R \rightarrow S_{n}$ for $n \geq 0$ such that $u^{0}$ is the canonical surjection $R \rightarrow R_{0}$ followed by $v$. As $R$ is quasi-free and $S_{n} \rightarrow S_{n-1}$ are square-zero extensions, such a family can be constructed inductively by choosing $u^{n}$ to be a lifting of $u^{n-1}$.

(ii) Let $u_{0}^{n}, u_{1}^{n}: R \rightarrow S_{n}$ be the families corresponding to $u_{0}, u_{1}$. The desired homomorphism $u_{t}$ is equivalent to a compatible family of homomorphisms $u_{t}^{n}: R \rightarrow S_{n}[t]$ such that $u_{t}^{n}$ joins $u_{0}^{n}, u_{1}^{n}$, and such that $u_{t}^{0}$ is $R \rightarrow R_{0}$ followed by $v_{t}$. Such a family can be constructed inductively by applying the preceding lemma successively to the square-zero extensions $S_{n} \rightarrow S_{n-1}$.

The following is analogous to the key step in the construction of derived functors.

Theorem 9.3. Let $A=R / I, B=S / J$ be algebra extensions with $R$ quasifree. Then any homomorphism $v: A \rightarrow B$ can be lifted to a homomorphism $u:\left(R_{n}\right) \rightarrow\left(S_{n}\right)$, and the induced map $u_{*}: \mathscr{X}(R, I) \rightarrow \mathscr{X}(S, J)$ modulo homotopy depends only on $v$.

Proof. This is a consequence of 8.7 and 9.2. Observe that if $u_{0}, u_{1}$ are two liftings of $v$, then by applying 9.2(ii) in the case of the constant homotopy $v_{t}=$ $v$ we see that $u_{0}, u_{1}$ are joined by a restricted family $u_{t}$. Thus $8.7(\mathrm{~b})$ applies with $\varepsilon=0$, and we have an odd degree map of towers $H^{\phi}\left(u_{t}\right): \mathscr{X}(R, I) \rightarrow$ $\mathscr{Z}(S, J)$ which is a homotopy between $u_{0 *}, u_{1 *}$.

This result implies by well-known arguments that for any quasi-free extension $A=R / I$, i.e., with $R$ quasi-free, the tower $\mathscr{X}(R, I)$ is determined up to homotopy equivalence by $A$, and moreover that modulo homotopy it is a functor of $A$. We can express this idea in the following way using the universal extension. Let $R_{n} A=R A / I A^{n+1}$, and recall that $\mathscr{X}_{A}=\mathscr{X}(R A, I A)$.

Corollary 9.4. (a) For any extension $A=R / I$ there is a canonical map

$$
\mathscr{X}_{A} \stackrel{w}{\rightarrow} \mathscr{X}(R, I)
$$

in the homotopy category of towers $H o \mathscr{T}$, which is obtained by choosing any homomorphism $\left(R_{n} A\right) \rightarrow\left(R_{n}\right)$ lifting the identity map $A \rightarrow R / I$ and taking 
the induced map on $\mathscr{X}$ towers. When $R$ is quasi-free $w$ is an isomorphism in Hog.

(b) Given extensions $A^{\prime}=R^{\prime} / I^{\prime}, A=R / I$, a homomorphism $v: A^{\prime} \rightarrow A$, and a homomorphism $u:\left(R_{n}^{\prime}\right) \rightarrow\left(R_{n}\right)$ lifting $v$, we have a commutative square in $H o \mathscr{T}$ :

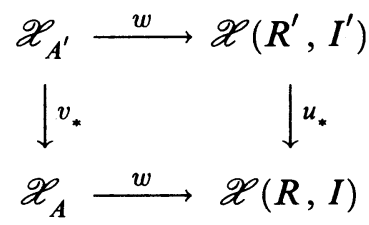

This is a straightforward consequence of the theorem.

Combining (a) with $\S 6(9)$ we obtain for any quasi-free extension $A=R / I$ a canonical isomorphism

$$
\theta \Omega A \simeq \mathscr{X}(R, I)
$$

in the homotopy category of towers $\operatorname{Ho\mathscr {T}}$. Thus $\mathscr{X}(R, I)$ is a special tower which represents the cyclic homology type of $A$ in the sense of the discussion at the end of $\S 2$. In particular the homology associated to this tower is given by the cyclic type homology of $A$.

We next apply 9.4 to derive some key results in [Q2] which were originally obtained by means of spectral sequences.

Consider an arbitrary extension $A=R / I$, and following the Hochschild tradition choose a linear lifting $\rho: A \rightarrow R$ respecting identity elements. By the universal property of $R A$ the lifting $\rho$ gives rise to a homomorphism $\left(R_{n} A\right) \rightarrow$ $\left(R_{n}\right)$ lifting the identity map of $A$. Hence by 9.4 we get a map of towers $\mathscr{X}_{A}^{n}=\mathscr{X}(R A, I A) \rightarrow \mathscr{X}(R, I)$ whose homotopy class $w$ is independent of the choice of $\rho$. From

$$
\begin{aligned}
H C_{2 n} A & =H_{+}\left(\mathscr{X}_{A}^{2 n}\right) \\
& \stackrel{w}{\rightarrow} H_{+}\left(\mathscr{X}^{2 n}(R, I)\right) \\
& =H_{+}\left(R / I^{n+1}+\left[I^{n}, R\right] \leftrightarrows \Omega^{1} R_{\natural} / \natural\left(I^{n} d R\right)\right) \\
& =\operatorname{Ker}\left(R / I^{n+1}+[R, R] \rightarrow \Omega^{1} R_{\natural} / \natural\left(I^{n} d R\right)\right)
\end{aligned}
$$

where the first line comes from $\S 6(10)$, we obtain a canonical map

$$
\gamma: H C_{2 n} A \rightarrow R / I^{n+1}+[R, R]=H C_{0}\left(R / I^{n+1}\right)
$$

In terms of the cochain notation of $\S 6$ this map can be described dually as carrying a trace $\tau$ on $R / I^{n+1}$ to the class of the even cochain $f$ with components

$$
f_{2 n}\left(a_{0}, \ldots, a_{2 n}\right)=\frac{(-1)^{n}}{n !} \tau\left(\rho\left(a_{0}\right) \omega\left(a_{1}, a_{2}\right) \cdots \omega\left(a_{2 n-1}, a_{2 n}\right)\right)
$$

where $\omega$ is the curvature $\S 5(4)$. Thus we find that $f$ is a $b+B$ cocycle whose cohomology class is independent of the choice of $\rho$. Previous versions of this type of result can be found in [Q1, Theorem 2, p. 148], [Q2, Theorem 1, p. 225].

When $R$ is quasi-free the arrow $w$ in (4) is an isomorphism, so we obtain the first exact sequence of the following proposition; cf. [Q2, I, 5.14]. 
Proposition 9.5. Given an extension $A=R / I$, then if $R$ is quasi-free we have an exact sequence

$$
0 \rightarrow H C_{2 n} A \stackrel{\vartheta}{\rightarrow} H C_{0}\left(R / I^{n+1}\right) \stackrel{\text { qd }}{\rightarrow} \Omega^{1} R_{\natural} /\left\lfloor\left(I^{n} d R\right)\right.
$$

and if $R$ is free we have exact sequences

$$
\begin{gathered}
0 \rightarrow H C_{2 n+1} A \rightarrow I^{n+1} /\left[I^{n}, I\right] \rightarrow R /[R, R], \\
0 \rightarrow \bar{H} C_{2 n+1} A \rightarrow I^{n+1} /\left[I^{n}, I\right] \rightarrow R / \mathbb{C}+[R, R] .
\end{gathered}
$$

We note that the exact sequences (8) and (9) have the following dual interpretations. Define an $I$-adic trace on $I^{n+1}$ to be a linear functional which vanishes on $\left[I^{i}, I^{k}\right]$ for $i+k=n+1$. From the identity

$$
\left[x, y_{1} \cdots y_{k}\right]=\sum_{i=1}^{k}\left[y_{i+1} \cdots y_{k} x y_{1} \cdots y_{i-1}, y_{i}\right]
$$

obtained by iterating the Hochschild 1-cocycle identity $\S 4(8)$ for $f(x, y)=$ $[x, y]$, we deduce $\left[I^{j}, I^{k}\right] \subset\left[I^{j+k-1}, I\right]$ for $k \geq 1$. Hence a linear functional on $I^{n+1}$ is an $I$-adic trace iff it vanishes on $\left[I^{n}, I\right]$. Then (8) and (9) identify elements of $H C^{2 n+1} A$ and $\bar{H} C^{2 n+1} A$ with $I$-adic traces on $I^{n+1}$ modulo the restrictions of traces and reduced traces on $R$ respectively.

To prove (8) we consider for an arbitrary extension the composition of canonical maps

$$
\begin{aligned}
H C_{2 n+1} A & =H_{-}\left(\mathscr{X}_{A}^{2 n+1}\right) \\
& \stackrel{w}{\rightarrow} H_{-}\left(\mathscr{X}^{2 n+1}(R, I)\right) \\
& =H_{-}\left(R / I^{n+1} \leftrightarrows \Omega^{1} R_{\natural} / \mathfrak{h}\left(I^{n+1} d R+I^{n} d I\right)\right) \\
& =\operatorname{Ker}\left(\Omega^{1} R_{\natural} / \mathfrak{h}\left(I^{n+1} d R+I^{n} d I+d R\right) \rightarrow R / I^{n+1}\right) \\
& \stackrel{\bar{b}}{\rightarrow} \operatorname{Ker}\left([R, R] /\left[I^{n}, I\right] \rightarrow R / I^{n+1}\right) \\
& =\left([R, R] \cap I^{n+1}\right) /\left[I^{n}, I\right]
\end{aligned}
$$

where the arrow labeled $\bar{b}$ is induced by the surjection

$$
\bar{b}: \Omega^{1} R_{\natural} /\lfloor(d R) \rightarrow[R, R], \quad \natural(x d y) \mapsto[x, y]
$$

and we have used the inclusion $\left[I^{n+1}, R\right] \subset\left[I^{n}, I\right]$. When $R$ is free, (11) is an isomorphism since the odd degree homology of $X(R)$ vanishes. Thus both the arrows $w$ and $\bar{b}$ in (10) are isomorphisms, yielding the exact sequence (8).

In deriving 9.3 and 9.4 we used the homotopy property 8.7 of the tower $\mathscr{Z}(R, I)$ and the homotopy lifting property 9.2 for quasi-free algebras. However, a simpler version of our arguments using instead 8.4 and 9.1 shows that for an arbitrary extension $A=R / I$ there is a canonical map modulo homotopy

$$
\left(F_{I A}^{q} X(R A)\right) \stackrel{w}{\rightarrow}\left(F_{I}^{q} X(R)\right)
$$


which is a homotopy equivalence when $R$ is free. Consequently we have a canonical map

$$
\begin{aligned}
\bar{H} C_{2 n+1} A & =H_{+}\left(F_{I A}^{2 n+1} X(R A)\right) \\
& \stackrel{w}{\rightarrow} H_{+}\left(F_{I}^{2 n+1} X(R)\right) \\
& =H_{+}\left(I^{n+1} \leftrightarrows \natural\left(I^{n+1} d R+I^{n} d I\right)\right) \\
& =\operatorname{Ker}\left(I^{n+1} /\left[I^{n}, I\right] \stackrel{\natural d}{\rightarrow} \Omega^{1} R_{\natural}\right)
\end{aligned}
$$

where the first line comes from $\S 6(8)$. When $R$ is free this map is an isomorphism, yielding the exact sequence $[\mathrm{Q} 2, \mathrm{I}, 5.12]$

$$
0 \rightarrow \bar{H} C_{2 n+1} A \rightarrow I^{n+1} /\left[I^{n}, I\right] \stackrel{\natural d}{\rightarrow} \Omega^{1} R_{\natural} .
$$

Moreover, as $R$ is free we have $H_{+}(X(R))=\mathbb{C}$, so the kernel of $\left\lfloor d: R \rightarrow \Omega^{1} R_{\natural}\right.$ is $\mathbb{C}+[R, R]$. This gives the exact sequence (9), concluding the proof of 9.5 .

Finally we mention that the formula $\S 6(18)$ for the homology of $X\left(R / I^{n+1}\right)$ in the case of the universal extension generalizes to an arbitrary quasi-free extension $A=R / I$ in the form

$$
\begin{aligned}
& H D_{0}\left(R / I^{n+1}\right)=H D_{2 n} A, \\
& H C_{1}\left(R / I^{n+1}\right)=H C_{2 n+1} A \oplus(1-\sigma) W
\end{aligned}
$$

where $W=\left[\left(I / I^{2}\right) \otimes_{A}\right]^{(n+1)}$ is the circular tensor product of order $n+1$ of the $A$-bimodule $I / I^{2}$, and $\sigma$ is the forward shift cyclic permutation. This is proved in the last section of [Q3].

\section{GOODWILLIE'S THEOREM AND BIVARIANT PERIODIC CYCLIC HOMOLOGY}

As another application of 9.4 we derive a strong form of the theorem of Goodwillie [G] about the invariance of periodic cyclic homology under nilpotent extensions.

Let $A^{\prime} \rightarrow A$ be a nilpotent extension, and let $R$ be any quasi-free algebra mapping onto $A^{\prime}$. Then we have $A^{\prime}=R / J, A=R / I$ where $J, I$ are ideals in $R$ such that $I^{m} \subset J \subset I$ for some $m$. Let $v: A^{\prime} \rightarrow A$ and $u:\left(R / J^{n+1}\right) \rightarrow$ $\left(R / I^{n+1}\right)$ be the obvious maps.

From 9.4 we obtain a homotopy commutative square of towers

$$
\begin{aligned}
& \mathscr{X}_{A^{\prime}} \longrightarrow \mathscr{X}(R, J) \\
& \mathscr{X}_{A} \longrightarrow \mathscr{X}(R, I)
\end{aligned}
$$

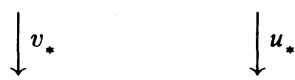

where the horizontal arrows are homotopy equivalences. Applying the inverse limit functor we obtain a homotopy commutative square of topological supercomplexes such that both horizontal arrows are homotopy equivalence of topological supercomplexes, by which we mean that the homotopy inverse map and 
the homotopy operators joining the two compositions to the identity are continuous.

Now $\widehat{\mathscr{X}}(R, J)$ is the completion of $X(R)$ for the topology defined by the filtration $\operatorname{Ker}\left\{X(R) \rightarrow X\left(R / J^{n}\right)\right\}$, and similarly for $\widehat{\mathscr{X}}(R, I)$. As the ideals $I, J$ contain powers of each other, the topologies coincide, so the map on inverse limits induced by $u_{*}$ is an isomorphism of topological supercomplexes.

From these observations we deduce at once

Theorem 10.1. If $A^{\prime} \rightarrow A$ is a nilpotent extension, then the induced map $\widehat{\mathscr{X}}_{A^{\prime}} \rightarrow$ $\widehat{\mathscr{X}}_{A}$ is a homotopy equivalence of topological supercomplexes. In particular, we have $H P_{\nu} A^{\prime} \stackrel{\sim}{\rightarrow} H P_{\nu} A$.

We turn next to bivariant groups associated to periodic cyclic homology. There are two natural candidates for bivariant periodic cyclic cohomology we might consider. On one hand, one can follow Jones and Kasse [JK] and make bivariant cyclic cohomology periodic by inverting the $S$ operation. On the other hand, one can consider the supercomplexes calculating periodic cyclic homology, form an appropriate mapping supercomplex, and use this to obtain bivariant groups. We will take the latter for our definition, since it is better from the standpoint of Goodwillie's theorem, as we will see.

Given algebras $A, B$, we define the associated bivariant periodic cyclic cohomology groups by

$$
H P^{\nu}(A, B)=H_{\nu}\left(\operatorname{Hom}_{c}\left(\widehat{\mathscr{O}}_{A}, \widehat{\mathscr{X}}_{B}\right)\right)
$$

where $\operatorname{Hom}_{c}\left(\widehat{\mathscr{O}}_{A}, \widehat{\mathscr{X}}_{B}\right)$ is the space of continuous linear maps $\S 2(5)$. Thus an element of $H P^{+}(A, B)$ is a homotopy class of continuous maps $\widehat{\mathscr{X}}_{A} \rightarrow \widehat{\mathscr{X}}_{B}$ respecting the gradings and differentials, where the homotopy relation is defined using continuous homotopy operators. In particular a homomorphism $A \rightarrow B$ determines a class $[A \rightarrow B]$ in $H P^{+}(A, B)$. There is an obvious cup product operation

$$
H P^{\nu}(A, B) \otimes H P^{\nu^{\prime}}(B, C) \rightarrow H P^{\nu+\nu^{\prime}}(A, C)
$$

given by composition.

Using bivariant periodic cyclic cohomology we may formulate 10.1 as follows.

Corollary 10.2. Given a nilpotent extension $A^{\prime} \rightarrow A$, there is a class in $H P^{+}\left(A, A^{\prime}\right)$ which is the inverse of the class $\left[A^{\prime} \rightarrow A\right] \in H P^{+}\left(A^{\prime}, A\right)$ with respect to cup product. Equivalently, $A^{\prime} \rightarrow A$ induces isomorphisms on $\operatorname{HP}^{*}(-,-)$ with respect to either variable.

The inverse class in $\operatorname{HP}^{+}\left(A, A^{\prime}\right)$ will be called the Goodwillie class of the nilpotent extension.

We next consider the bivariant groups defined by making bivariant cyclic cohomology periodic. We have seen in $\S 2$ that the bivariant cyclic cohomology groups of Jones-Kassel may be expressed as

$$
H C^{k}(A, B)=H_{k+2 \mathbb{Z}}\left(\operatorname{Hom}^{k}\left(\mathscr{X}_{A}, \mathscr{X}_{B}\right)\right)
$$


where $\operatorname{Hom}^{k}\left(\mathscr{X}_{A}, \mathscr{Z}_{B}\right)$ is the subcomplex of $\operatorname{Hom}_{c}\left(\widehat{\mathscr{X}}_{A}, \widehat{\mathscr{X}}_{B}\right)$ consisting of the maps $f: \widehat{\mathscr{X}}_{A} \rightarrow \widehat{\mathscr{X}}_{B}$ of order $\leq k$, i.e., such that $f$ induces $f: \mathscr{Z}_{A}^{p} \rightarrow \mathscr{X}_{B}^{p-k}$ for all $p$. The inclusion of this subcomplex gives rise to a canonical map

$$
H C^{k}(A, B) \rightarrow H P^{k+2 \mathrm{Z}}(A, B)
$$

compatible with cup product.

Jones and Kassel consider the inductive limit of the bivariant cyclic cohomology under the $S$ operation. This yields the bivariant groups

$$
S^{-1} H C^{\nu}(A, B)=H_{\nu}\left(\operatorname{Hom}^{\infty}\left(\mathscr{X}_{A}, \mathscr{X}_{B}\right)\right)
$$

where $\mathrm{Hom}^{\infty}$ is the union of the $\mathrm{Hom}^{k}$.

We now ask whether the analogue of 10.2 holds with $S^{-1} H C^{*}$ in place of $H P^{*}$, in other words, whether for any nilpotent extension $A^{\prime} \rightarrow A$ there exists a class in $S^{-1} H C^{+}\left(A, A^{\prime}\right)$ inverse to the class of the homomorphism $A^{\prime} \rightarrow A$. In order to investigate this question we introduce the following terminology.

We will say that the nilpotent extension $A^{\prime} \rightarrow A$ has size $\leq n$, where $n$ is an integer $\geq 0$, if there exists a homotopy inverse for the induced map $\widehat{\mathscr{X}}_{A^{\prime}} \rightarrow \widehat{\mathscr{X}}_{A}$ having order $\leq 2 n$. This is equivalent to the Goodwillie class being in the image of the map $H C^{2 n}\left(A, A^{\prime}\right) \rightarrow H P^{+}\left(A, A^{\prime}\right)$. Define the size $s\left(A^{\prime} \rightarrow A\right)$ to be the least such $n$ if one exists, and set $s\left(A^{\prime} \rightarrow A\right)=\infty$ otherwise.

It is clear that if a class exists in $S^{-1} H C\left(A, A^{\prime}\right)$ inverse to the class of the homomorphisms $A^{\prime} \rightarrow A$, then the size is necessarily finite. We are going to prove the existence of nilpotent extensions of infinite size, thereby showing that the analogue of 10.2 does not hold for $S^{-1} H C^{*}$.

We begin by showing the size of a nilpotent extension can be as big as its order. Recall that $R_{n} A=R A / I A^{n+1}$ is the universal nilpotent extension of $A$ of order $\leq n$.

Lemma 10.3. For any $n \geq 0$ there exists an algebra $A$ such that $s\left(R_{n} A \rightarrow A\right) \geq$ $n$.

Proof. Consider an arbitrary algebra $A$, put $R=R A, I=I A, R_{n}=R_{n} A$, and let $m=s\left(R_{n} \rightarrow A\right)$. There is nothing to prove if $m \geq n$, so suppose $m<n$. By the definition of size the induced map $\widehat{\mathscr{X}}_{R_{n}} \rightarrow \widehat{\mathscr{X}}_{A}$ has a homotopy inverse of order $\leq 2 \mathrm{~m}$. The proof of 10.1 shows that up to homotopy equivalence we can identify this induced map with the isomorphism

$$
\widehat{\mathscr{X}}\left(R, I^{n+1}\right) \stackrel{\sim}{\rightarrow} \widehat{\mathscr{X}}(R, I)
$$

of completions of $X(R)$. Thus if $g$ denotes the inverse isomorphism, we know that $g$ is homotopic to a map $f$ of order $\leq 2 m$.

Note that $F_{I}^{2 n} X(R) \subset F_{I^{n+1}}^{0} X(R)$, hence we obtain the commutative square on the left

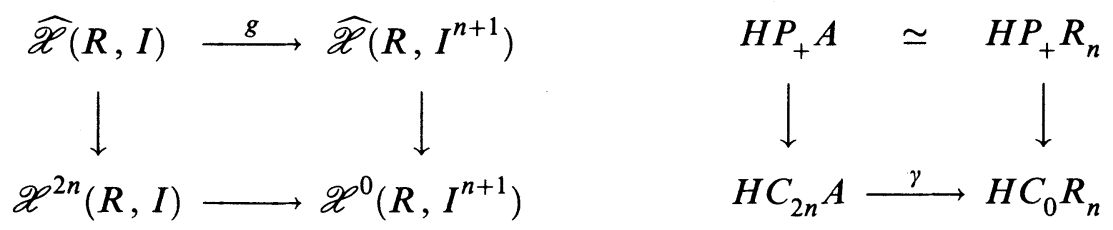


where the vertical arrows are the canonical surjections. Taking homology of even degree yields the square on the right, where the vertical arrows are the canonical maps from periodic cyclic to cyclic homology. The bottom arrow is easily seen to be the map $\gamma^{\prime}$ of $\S 9$ (7), hence it is injective.

On the other hand, because $f$ has order $\leq 2 m$ we get similar commutative squares:

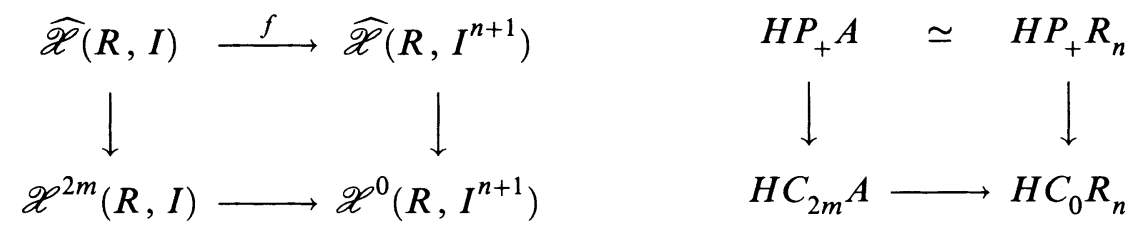

Now the two squares on the right have the same top and right arrows. Using the injectivity of $\gamma$ we see that

$$
\operatorname{Ker}\left(H P_{+} A \rightarrow H C_{2 n} A\right)=\operatorname{Ker}\left(H P_{+} A \rightarrow H C_{2 m} A\right) .
$$

But it is easy to produce algebras $A$ such that this is false, for example, the Weyl algebra with $2 n$ generators, which has $H P_{+} A=H C_{2 k} A=\mathbb{C}$ for $k \geq n$ and $H C_{2 k} A=0$ for $k<n$. One also has a smooth commutative example given by the tensor product of $2 n$ copies of the Laurent polynomial algebra $\mathbb{C}\left[z, z^{-1}\right]$. For such an $A$ the size of the extension $R_{n}$ is $\geq n$, which proves the lemma.

The next two lemmas establish some formal properties of the size.

Lemma 10.4. (i) If $A^{\prime} \rightarrow A$ and $A^{\prime \prime} \rightarrow A$ are nilpotent extensions and there exists a homomorphism $A^{\prime} \rightarrow A^{\prime \prime}$ over $A$, then $s\left(A^{\prime} \rightarrow A\right) \geq s\left(A^{\prime \prime} \rightarrow A\right)$.

(ii) If $A^{\prime \prime} \rightarrow A^{\prime}$ and $A^{\prime} \rightarrow A$ are nilpotent extensions, then

$$
s\left(A^{\prime \prime} \rightarrow A\right) \leq s\left(A^{\prime \prime} \rightarrow A^{\prime}\right)+s\left(A^{\prime} \rightarrow A\right) .
$$

Proof. This is straightforward from the definition.

Lemma 10.5. Let $p: B \rightarrow A$ be a homomorphism of unital algebras, and let $i: A \rightarrow B$ be a homomorphism of the underlying nonunital algebras such that $p i$ is the identity map on $A$. Then $s\left(R_{1} A \rightarrow A\right) \leq s\left(R_{1} B \rightarrow B\right)$.

Proof. Let $\pi_{A}: R_{1} A \rightarrow A$ denote the canonical surjection, and similarly for $B$. We can assume $n=s\left(R_{1} B \rightarrow B\right)$ is finite, whence there exists a homotopy inverse $f$ for $\pi_{B *}: \widehat{\mathscr{X}}_{R_{1} B} \rightarrow \widehat{\mathscr{X}}_{B}$ having order $\leq 2 n$.

We consider first the case where $i: A \rightarrow B$ is a unital homomorphism. The composition

$$
\widehat{\mathscr{X}}_{A} \stackrel{i}{\rightarrow} \widehat{\mathscr{X}}_{B} \stackrel{f}{\rightarrow} \widehat{\mathscr{X}}_{R_{1} B} \stackrel{\left(R_{1} p\right)}{\longrightarrow} \widehat{\mathscr{X}}_{R_{1} A}
$$

has order $\leq 2 n$ and satisfies $\pi_{A *}\left(R_{1} p\right)_{*} f i_{*}=p_{*} \pi_{B *} f i_{*} \sim p_{*} i_{*}=1$. Since $\pi_{A *}$ is a homotopy equivalence, this composition is necessarily a homotopy inverse for $\pi_{A *}$. Thus we have $s\left(R_{1} A \rightarrow A\right) \leq n$, which was to be proved.

The same argument works in general because cyclic homology theory can be defined on the category of nonunital algebras; cf. [K2]. Thus a nonunital homomorphism $i: A \rightarrow B$ determines an induced map $\mathscr{X}_{A} \rightarrow \mathscr{X}_{B}$ defined 
modulo homotopy, or equivalently, a class in $H C^{0}(A, B)$. To be more specific, we note that $i: A \rightarrow B$ is equivalent to a unital aigebra homomorphism $i_{\#}: \widetilde{A} \rightarrow$ $B$, where $\tilde{A}$ is the algebra obtained by regarding $A$ as a nonunital algebra and adjoining an identity element. Moreover we can identify $\tilde{A}$ with the product algebra $\mathbb{C} \times A$. Thus we have maps

$$
\mathscr{X}_{A} \stackrel{(0,1)}{\longrightarrow} \mathscr{X}_{\mathbb{C}} \times \mathscr{X}_{A} \leftarrow \mathscr{X}_{\mathbb{C} \times A} \stackrel{i_{* *}}{\longrightarrow} \mathscr{X}_{B}
$$

where the middle arrow is a homotopy equivalence by the additivity of cyclic homology for a product of two algebras. Using this we obtain a map $i_{*}: \mathscr{X}_{A} \rightarrow$ $\mathscr{X}_{B}$ defined modulo homotopy, which satisfies $p_{*} i_{*} \sim 1$ as one easily checks. Then the induced map on the inverse limits $i_{*}: \mathscr{\mathscr { X }}_{A} \rightarrow \widehat{\mathscr{X}}_{B}$ also satisfies $p_{*} i_{*} \sim$ 1 , permitting the argument given above in the unital case to proceed.

Proposition 10.6. There exists an algebra $A$ such that $s\left(R_{1} A \rightarrow A\right)=\infty$, i.e., such that the Goodwillie class in $\operatorname{HP}^{+}\left(A, R_{1} A\right)$ does not come from $S^{-1} H C^{+}\left(A, R_{1} A\right)$.

Proof. Put

$$
m=\sup _{A} s\left(R_{1} A \rightarrow A\right) .
$$

Since $R_{1} A \rightarrow A$ maps to any square-zero extension of $A$, it follows from 10.4(i) that any square-zero extension has size $\leq m$. Then 10.4(ii) implies for any extension $A=R / I$ and integer $p \geq 0$ that

$$
s\left(R / I^{2^{p}} \rightarrow A\right) \leq \sum_{j=1}^{p} s\left(R / I^{2^{j}} \rightarrow R / I^{2^{j-1}}\right) \leq p m .
$$

Combining this with 10.3 in the case $n=2^{p}-1$, we obtain for each $p$ an algebra $A$ such that

$$
2^{p}-1 \leq s\left(R A / I A^{2^{p}} \rightarrow A\right) \leq p m .
$$

This implies $m=\infty$, i.e., there exists a sequence of algebras $A_{n}$ such that $s\left(R_{1} A_{n} \rightarrow A_{n}\right)$ tends to infinity.

Let $B$ be any algebra such that

$$
\mathbb{C} \oplus \bigoplus_{n} A_{n} \subset B \subset \prod_{n} A_{n}
$$

and let $p_{n}: B \rightarrow A_{n}$ and $i_{n}: A_{n} \rightarrow B$ denote the obvious projections and injections. Note that $p_{n}$ is a homomorphism of unital algebras and $i_{n}$ is a homomorphism of the underlying nonunital algebras such that $p_{n} i_{n}$ is the identity. By 10.5 we have $s\left(R_{1} B \rightarrow B\right) \geq s\left(R_{1} A_{n} \rightarrow A_{n}\right)$ for all $n$, so $s\left(R_{1} B \rightarrow B\right)=\infty$.

\section{Nistor's Bivariant Chern ChaRACTER}

The problem of associating a bivariant Chern character in $H C^{*}(A, B)$ with certain (" $p$-summable") cycles for the bivariant $K$-theory $K K(A, B)$ has been studied by several authors including Wang and Kassel. The most useful and elegant results in this direction have been obtained by Nistor [N]. We now apply 
our framework to construct in a simple way a bivariant Chern character of the same type as Nistor's but with better bounds. One can show our construction is essentially equivalent to Nistor's, but this point will not be discussed here.

Let $A, B, L$ be algebras and let $J \subset L$ be an ideal. Let $\tau$ be a $J$-adic trace on $J^{p}$, that is, a linear functional vanishing on $\left[J^{i}, J^{j}\right]$ for $i+j=p$. In applications to Fredholm modules $L, J, \tau$ are respectively the algebra of bounded operators on Hilbert space, the Schatten ideal of $p$-summable operators, and the canonical trace on trace class operators.

Suppose given a quasi-homomorphism from $A$ to $B$ consisting of two homomorphisms $\varphi, \bar{\varphi}: A \rightarrow L \otimes B$ which are congruent modulo the ideal $J \otimes B$. Improving on Nistor's result slightly, we will construct elements

$$
C h^{2 m}(\varphi, \bar{\varphi}, \tau) \in H C^{2 m}(A, B)
$$

for $2 m \geq p-1$ (rather than $m \geq p-1$ ) such that $C h^{2 m+2}(\varphi, \bar{\varphi}, \tau)=$ $\operatorname{SCh}^{2 m}(\varphi, \bar{\varphi}, \tau)$.

We begin with some preliminary constructions. Let

$$
J_{\#}^{n}=J^{n} / \sum_{i=0}^{n}\left[J^{i}, J^{n-i}\right]
$$

and let $\#_{n}: J^{n} \rightarrow J_{\#}^{n}$ be the canonical surjection. Then $\#_{n}$ is the universal $J$ adic trace on $J^{n}$ with values in a vector space.

Let $S$ be the graded algebra

$$
S=\bigoplus_{n \geq 0} t^{n} J^{n} \subset \mathbb{C}[t] \otimes L
$$

where $t$ is an indeterminate. Then

$$
S_{\natural}=\bigoplus_{n \geq 0} t^{n} J_{\#}^{n}
$$

so that a $J$-adic trace on $J^{n}$ is equivalent to a homogeneous trace of degree $n$ on $S$. Let

We next define certain inhomogeneous traces $\mu_{m}: S \rightarrow J_{\#}^{2 m+1}$ for $m \geq 0$.

$$
P_{m}(z)=\prod_{k=1}^{m}\left(1-\frac{z}{2 k-1}\right)
$$

and define $\mu_{m}$ by

$$
\mu_{m}\left(t^{n} x\right)=\frac{1}{2}\left(1-(-1)^{n}\right) P_{m}(n) \#_{2 m+1}(x), \quad x \in J^{n} .
$$

This is well defined since the numerical function of $n$ vanishes for $0 \leq n \leq 2 m$.

Lemma 11.1. (i) $\mu_{m}$ is a trace on $S$.

(ii) $\mu_{m}$ vanishes on $K^{m+1}$, where $K$ is the ideal in $S$ generated by $\left(1-t^{2}\right) J^{2}$. 
(iii) One has a commutative square

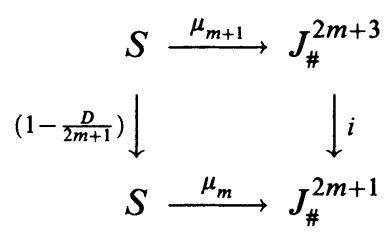

where $D=t \frac{d}{d t}$ is the degree operator on $S$ and $i$ is the map induced by the inclusion $J^{2 m+3} \subset J^{2 m+1}$.

Proof. It is clear from (2) that $\mu_{m}$ descends to $S_{\mathrm{h}}$, so (i) holds.

We can also describe $\mu_{m}$ as the composition of the map

$$
\frac{1}{2}\left(\delta_{1}-\delta_{-1}\right) P_{m}(D): S \rightarrow J^{2 m+1}
$$

where $\delta_{ \pm 1}$ evaluates a polynomial in $t$ at \pm 1 , followed by $\#_{2 m+1}$. The assertion (iii) follows immediately from this description.

Finally as $D$ is a derivation of $S$ and $P_{m}(D)$ has degree $m$ in $D$, we see that $P_{m}(D)$ maps $K^{m+1}$ into $K=\sum_{n \geq 0}\left(1-t^{2}\right) t^{n} J^{2+n}$, which is killed by $\delta_{ \pm 1}$, proving (ii).

Consider now the quasi-homomorphism $\varphi, \bar{\varphi}: A \rightarrow L \otimes B$ and set

$$
\begin{aligned}
& p=\frac{1}{2}(\varphi+\bar{\varphi}): A \rightarrow L \otimes H, \\
& q=\frac{1}{2}(\varphi-\bar{\varphi}): \bar{A} \rightarrow J \otimes H .
\end{aligned}
$$

Then $p+t q: A \rightarrow S \otimes B$ is a linear map respecting identity elements, whose curvature is

$$
a_{1} \otimes a_{2} \mapsto\left(1-t^{2}\right) q a_{1} q a_{2}: \bar{A}^{\otimes 2} \rightarrow\left(1-t^{2}\right) J^{2} \otimes B \subset K \otimes B .
$$

By the universal property 5.1 of $R A$ there is a unique homomorphism $u: R A$ $\rightarrow S \otimes R B$ such that

$$
u \rho_{A}=\left(1 \otimes \rho_{B}\right)(p+t q)
$$

where $\rho_{A}$ denotes the canonical inclusion of $A$ as a subspace of $R A$ and similarly for $\rho_{B}$. Since $p+t q: A \rightarrow(S / K) \otimes B$ is a homomorphism, we have

$$
u(I A) \subset K \otimes R B+S \otimes I B .
$$

Let $\chi_{m}$ denote the composition

$$
X(R A) \stackrel{u_{*}}{\rightarrow} X(S \otimes R B) \stackrel{\alpha}{\rightarrow} S_{\mathrm{h}} \otimes X(R B) \stackrel{\mu_{m}}{\rightarrow} J_{\#}^{2 m+1} \otimes X(R B)
$$

where $\mu_{m}$ strictly speaking is $\mu_{m} \otimes 1$. We now examine the behavior of $\chi_{m}$ with respect to the canonical filtration $F_{I A}^{p}=F_{I A}^{p} X(R A)$ and similarly for $B$.

Proposition 11.2. The map $\chi_{m}: X(R A) \rightarrow J_{\#}^{2 m+1} \otimes X(R B)$ respects the supercomplex structure and has order $\leq 2 m$, i.e., it maps $F_{I A}^{p}$ to $J_{\#}^{2 m+1} \otimes F_{I B}^{p-2 m}$ for 
all $p$. There exists an odd map $h: X(R A) \rightarrow J_{\#}^{2 m+1} \otimes X(R B)$ of order $\leq 2 m+2$ such that $[\partial, h]=\chi_{m}-i \chi_{m+1}$.

Proof. The first assertion is clear from

$$
\begin{aligned}
F_{I A}^{p} & \stackrel{u_{*}}{\rightarrow} F_{K \otimes R B+S \otimes I B}^{p} \\
& \stackrel{\alpha}{\rightarrow} \sum_{i \geq 0} \natural\left(K^{i}\right) \otimes F_{I B}^{p-2 i} \\
& \stackrel{\mu_{m}}{\rightarrow} J_{\#}^{2 m+1} \otimes F_{I B}^{p-2 m} \quad(4.4) \\
& \left(\mu_{m}\left(K^{m+1}\right)=0\right) .
\end{aligned}
$$

For the second assertion we extend $D$ to $S \otimes R B$ and $S_{\natural} \otimes X(R B)$ as $D \otimes 1$. Then

$$
D u: R A \rightarrow S \otimes R B \rightarrow S \otimes R B
$$

is a derivation with respect to $u$, and one has a Lie derivative $L(u, D u)$ fitting into a commutative diagram:

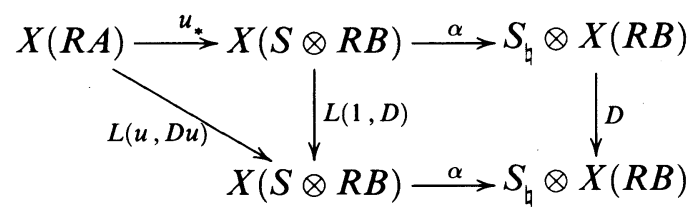

By 7.2 there is an odd map $h^{\phi}(u, D u): X(R A) \rightarrow X(S \otimes R B)$ such that

$$
L(u, D u)=\left[\partial, h^{\phi}(u, D u)\right]
$$

The existence of $\phi$ as in 7.1(b) follows from the fact that $R A$ is a free algebra. In fact there is a canonical choice for $\phi$, characterized by $\phi \rho_{A}=0$.

Using 11.1 (iii) we have

$$
\begin{aligned}
\chi_{m}-i \chi_{m+1} & =\left(\mu_{m}-i \mu_{m+1}\right) \alpha u_{*} \\
& =\frac{1}{2 m+1} \mu_{m} D \alpha u_{*} \\
& =\frac{1}{2 m+1} \mu_{m} \alpha L(u, D u) \\
& =[\partial, h]
\end{aligned}
$$

where $h=\frac{1}{2 m+1} \mu_{m} \alpha h^{\phi}(u, D u)$. By the first case of 7.4 we see that $h^{\phi}(u, D u)$ maps $F_{I A}^{p}$ to $F_{K \otimes R B+S \otimes I B}^{p-2}$, which we have already seen is mapped by $\mu_{m} \alpha$ to $J_{\#}^{2 m+1} \otimes F_{I B}^{p-2-2 m}$. Thus $h$ has order $\leq 2 m+2$.

Let $\tau$ be a $J$-adic trace on $J^{2 m+1}$, and let $\tau \cdot \chi_{m}: X(R A) \rightarrow X(R B)$ be $\chi_{m}$ followed by the map from $J_{\#}^{2 m+1} \otimes X(R B)$ to $X(R B)$ induced by $\tau$. By the first statement of the preceding proposition $\tau \cdot \chi_{m}$ is a map of supercomplexes having order $\leq 2 m$ with respect to the canonical filtrations. Thus it determines a map of special towers $\mathscr{X}_{A} \rightarrow \mathscr{X}_{B}[2 m]$, whose class in $H^{2 m}(A, B)$ is defined to be $C h^{2 m}(\varphi, \bar{\varphi}, \tau)$. 
By the second statement the difference $\tau \cdot \chi_{m}-\tau \cdot \chi_{m+1}$, where the latter map is defined using the restriction of $\tau$ to $J^{2 m+3}$, has the form $[\partial, \tau \cdot h]$, where $\tau \cdot h$ has order $\leq 2 m+2$. This shows that $S C h^{2 m}(\varphi, \bar{\varphi}, \tau)=C h^{2 m+2}(\varphi, \bar{\varphi}, \tau)$.

\section{ChERN CHARACTER}

In this section we use the description of periodic cyclic homology as the homology of $\widehat{\mathscr{X}}(R A, I A)$ in order to construct the canonical Chern character maps from $K_{i} A$ to $H P_{i+2 \mathbb{Z}} A$ for $i=0,1$. Given an extension $A=R / I$, one knows that idempotent and invertible matrices over $A$ can be lifted to the nilpotent extension $R / I^{n}$, and more generally to the $I$-adic completion $\widehat{R}$. We exploit this fact to define canonical maps from $K_{0} A$ and $K_{1} A$ to the homology of $\widehat{\mathscr{X}}(R, I)$ generalizing the familiar 'index' pairings, in which elements of $K_{0} A$ pair with traces on $R / I^{n}$, and elements of $K_{1} A$ pair with traces on $I^{n}$; cf. [Q2, II, §2]. In the case of the universal extension $A=R A / I A$ we obtain the Chern character maps from $K$-theory to periodic cyclic homology. The material in this section is a natural development of ideas introduced by Fedosov [F] in his version of the index theorem.

We begin by constructing canonical additive maps

$$
\begin{gathered}
K_{0} R \rightarrow \operatorname{Ker}\left\{R_{\natural} \stackrel{d}{\rightarrow} \Omega^{1} R_{\natural}\right\}=H D_{0} R, \quad[e] \mapsto \operatorname{ttr}(e), \\
K_{1} R \rightarrow \operatorname{Ker}\left\{\Omega^{1} R_{\natural} \stackrel{\bar{b}}{\rightarrow} R\right\}=H H_{1} R, \quad[g] \mapsto \operatorname{ttr}\left(g^{-1} d g\right) .
\end{gathered}
$$

For any vector space $V$ let $M_{k} V$ be the vector space of $k \times k$ matrices with entries in $V$, and for $x \in M_{k} V$ let $\operatorname{tr}(x) \in V$ be the sum of its diagonal entries. If $R$ is an algebra, then the maps

$$
\text { দtr: } M_{k} R \rightarrow R_{\mathrm{b}}, \quad \text { ttr: } M_{k} \Omega^{1} R \rightarrow \Omega^{1} R_{\mathrm{b}}
$$

are traces on the matrix algebra $M_{k} R$ and the $M_{k} R$ bimodule of matrix 1forms respectively.

The Grothendieck group $K_{0} R$ may be defined as the abelian group with generators $[e]$ for each idempotent matrix $e$ over $R$, subject to the relations:

(1) $[e]=\left[e^{\prime}\right]$ if $e^{\prime}=g e g^{-1}$ in $M_{k} R$.

(2) $\left[e \oplus e^{\prime}\right]=[e]+\left[e^{\prime}\right]$.

(3) $[e]=0$ if $e$ is the zero matrix in $M_{k} R$.

Using this definition we obtain easily an additive map $K_{0} R \rightarrow R_{\natural}$ sending [e] to $\operatorname{tat}(e)$.

Upon applying $d$ to $e^{2}=e$ we obtain $e d e=d e(1-e)$, dee $=(1-e) d e$, hence

$$
\natural \operatorname{tr}(e d e)=\emptyset \operatorname{tr}\left(e^{2} d e\right)=\emptyset \operatorname{tr}(e d e(1-e))=\operatorname{\natural tr}((1-e) e d e)=0 .
$$

Similarly $\operatorname{tat}((1-e) d e)=0$, whence $\operatorname{tat}(d e)=0$. This shows that $\operatorname{tr}(e)$ is killed by $d: R_{\natural} \rightarrow \Omega^{1} R_{\natural}$, so we obtain the map (1).

Next recall that $K_{1} R$ is the inductive limit of the groups of invertible matrices $G L_{k} R$ made abelian. If $g \in G L_{k} R$, then we have a corresponding class $[g] \in$ 
$K_{1} R$ and a matrix 1-form $g^{-1} d g \in M_{k} \Omega^{1} R$. Since

$$
\begin{aligned}
\operatorname{tat}\left(\left(g_{1} g_{2}\right)^{-1} d\left(g_{1} g_{2}\right)\right) & =\operatorname{tat}\left(g_{2}^{-1} g_{1}^{-1} d g_{1} g_{2}+g_{2}^{-1} d g_{2}\right) \\
& =\operatorname{ttr}\left(g_{1}^{-1} d g_{1}\right)+\operatorname{ttr}\left(g_{2}^{-1} d g_{2}\right)
\end{aligned}
$$

we obtain easily an additive map $K_{1} R \rightarrow \Omega^{1} R_{\natural}$ sending $[g]$ to $\operatorname{tr}\left(g^{-1} d g\right)$. As

$$
\bar{b}\left(\text { ttr }\left(g^{-1} d g\right)\right)=\operatorname{tr}\left(g^{-1} g\right)-\operatorname{tr}\left(g g^{-1}\right)=0
$$

this yields the map (2).

Lifting idempotents and invertibles. Let us consider an extension $A=R / I$, and let $\widehat{R}, \widehat{I}$ be the $I$-adic completions of $R, I$. We propose to construct canonical additive maps

$$
K_{i} A \rightarrow H_{i+2 \mathrm{Z}}(\widehat{\mathscr{X}}(R, I)), \quad i=0,1,
$$

by lifting idempotent and invertible matrices over $A$ to $\widehat{R}$, and then using the maps (1), (2) for $\widehat{R}$.

We begin with $K_{0} A$. The following is well known, but we give a proof in order to obtain a useful formula.

Lemma 12.1. An idempotent matrix e over $A$ lifts to an idempotent matrix over $\widehat{R}$ which is unique up to conjugation.

Proof. If $e \in M_{k} A$, then upon replacing the extension $A=R / I$ by the extension of matrix algebras $M_{k}=M_{k} R / M_{k} I$, we can assume $e \in A$.

It is easier if instead of the idempotent $e$ we work with the equivalent involution $2 e-1$. Let $z$ be any lifting of $2 e-1$ to an element of $\widehat{R}$. Then $z^{2}$ belongs to the group $1+\widehat{I}$ under multiplication. Because the exponential map $\widehat{I} \rightarrow 1+\widehat{I}$ is bijective, this group is uniquely divisible. Hence the inverse of $z^{2}$ has a unique square root $\left(z^{2}\right)^{-1 / 2}$ in this group. Then $s=z\left(z^{2}\right)^{-1 / 2}$ is a lifting of $2 e-1$ to an involution in $\widehat{R}$, and $\tilde{e}=\frac{1}{2}(1+s)$ is a lifting of $e$ to an idempotent.

To prove the uniqueness up to conjugacy, let $s^{\prime}$ be another involution in $\widehat{R}$ lifting $2 e-1$. Then $s^{\prime} s$ has a unique square root $u$ in $1+\widehat{I}$. Since $\left(s u^{-1} s\right)^{2}=s u^{-2} s=s s s^{\prime} s=u^{2}$, we have $s u^{-1} s=u$ by uniqueness. Then $u s u^{-1}=u^{2} s=s^{\prime}$, showing that $s, s^{\prime}$ are conjugate.

Let us now extract a formula for the idempotent $\tilde{e}$ lifting the given idempotent $e$ which is constructed in this proof. We have $z=2 x-1$, where $x$ is a lifting of $e$ to an element of $\widehat{R}$. The element $\left(z^{2}\right)^{1 / 2}$ is given by the binomial series

$$
\left(z^{2}\right)^{-1 / 2}=\left(1-\left(1-z^{2}\right)\right)^{-1 / 2}=\sum_{n \geq 0} \frac{1 \cdot 3 \cdots(2 n-1)}{2^{n} n !}\left(1-z^{2}\right)^{n} .
$$

As $1-z^{2}=4\left(x-x^{2}\right)$ we obtain

$$
\tilde{e}=\frac{1}{2}+\left(x-\frac{1}{2}\right) \sum_{n \geq 0} \frac{(2 n) !}{(n !)^{2}}\left(x-x^{2}\right)^{2 n} .
$$


This formula clearly holds more generally with matrices, so we obtain the following.

Lemma 12.2. Given an idempotent matrix $e \in M_{k} A$, let $x \in M_{k} \widehat{R}$ lift $e$. Then

$$
\tilde{e}=x+\left(x-\frac{1}{2}\right) \sum_{n \geq 1} \frac{(2 n) !}{(n !)^{2}}\left(x-x^{2}\right)^{2 n}
$$

is a lifting of $e$ to an idempotent matrix in $M_{k} \widehat{R}$.

It follows easily from the first lemma that we have an isomorphism $K_{0} \widehat{R} \simeq$ $K_{0} A$. Using (1) for $\widehat{R}$ we obtain a canonical map

$$
K_{0} A \rightarrow H D_{0} \widehat{R}=H_{+}(X(\widehat{R}))
$$

sending $[e]$ to $\operatorname{ttr}(\tilde{e})$, where $\tilde{e}$ is a lifting of $e$ to an idempotent matrix over $\widehat{R}$. Composing with the map on $H_{+}$induced by the obvious map $X(\widehat{R}) \rightarrow \widehat{\mathscr{X}}(R, I)$ then yields the desired map

$$
K_{0} A \rightarrow H_{+}(\widehat{\mathscr{X}}(R, I)) .
$$

We next discuss $K_{1} A$.

Lemma 12.3. If $g \in G L_{k} A$, then any lifting of $g$ to a matrix over $\widehat{R}$ is invertible. Proof. Let $p, q$ be arbitrary liftings of $g, g^{-1}$ respectively to matrices over $\widehat{R}$, and let $x=1-q p, y=1-p q$, so that $x, y$ have entries in $\widehat{I}$. Then $p q=1-y, q p=1-x$ are invertible with inverses given by geometric series. Thus $p, q$ are invertible, and

$$
p^{-1}=\sum_{n \geq 0} q y^{n}=\sum_{n \geq 0} x^{n} q
$$

since $p^{-1}=q(1-y)^{-1}=(1-x)^{-1} q$.

This lemma implies that $1+M_{k} \widehat{I}$ is a group under multiplication and that

$$
G L_{K} A=G L_{k} \widehat{R} / 1+M_{k} \widehat{I} \text {. }
$$

It follows that $K_{1} A$ is the quotient of $K_{1} \widehat{R}$ by the subgroup of classes [1-x] with $x$ a matrix over $\widehat{I}$.

We next use the map (2) for the algebra $\widehat{R}$ :

$$
K_{1} \widehat{R} \rightarrow \operatorname{Ker}\left(\Omega^{1}(\widehat{R})_{\natural} \stackrel{\bar{b}}{\rightarrow} \widehat{R}\right) .
$$

In order to obtain a map defined in $K_{1} A$, we must kill elements of the form

$$
\natural \operatorname{tr}\left((1-x)^{-1} d(1-x)\right)=- \text { ตtr }\left(\left(\sum_{n \geq 0} x^{n}\right) d x\right)
$$

where $x$ is a matrix over $\widehat{I}$. Consider the element of $\widehat{I}$,

$$
\operatorname{tr}(\log (1-x))=-\operatorname{tr}\left(\sum_{n \geq 0} \frac{x^{n+1}}{n+1}\right) .
$$


We have

$$
\natural d\left(\operatorname{tr}\left(\frac{x^{n+1}}{n+1}\right)\right)=\frac{1}{n+1} \sum_{i=0}^{n} \natural \operatorname{tr}\left(x^{i} d x x^{n-i}\right)=\operatorname{ttr}\left(x^{n} d x\right) .
$$

If we could take the infinite sum of these equations, we would have

$$
\natural d(\operatorname{tr}(\log (1-x)))=\operatorname{tr}\left((1-x)^{-1} d(1-x)\right)
$$

which would be surprising in view of the algebraic character of $\Omega^{1}(\widehat{R})_{4}$. However, what is clearly true is that this identity holds in $\Omega^{1}\left(R / I^{n}\right)_{b}$ for each $n$, since only finite sums are needed in this case. Thus we have a well-defined map

$$
K_{1} A \rightarrow \operatorname{Ker}\left(\lim ^{\lim } \Omega^{1}\left(R / I^{n}\right)_{\natural} \stackrel{b}{\rightarrow} \widehat{R}\right) / d \widehat{I}
$$

sending $[g]$ to $\operatorname{tr}\left(p^{-1} d p\right)$, where $p$ is any lifting of $g$ to a matrix over $\widehat{R}$. Upon dividing out by the larger space $d \widehat{R}$ on the right, we obtain the desired map

$$
K_{1} A \rightarrow H_{-}(\widehat{\mathscr{X}}(R, I)) .
$$

Clearly (5) gives a finer invariant for elements of $K_{1} A$ than (6). This is related to the fact that the map from algebraic $K$-theory to periodic cyclic theory factors through the negative cyclic theory $\mathrm{HC}^{-}$.

Pairing of $K$-classes with traces. Before proceeding to the universal extension, we discuss how the canonical maps (3) generalize the index pairings which pair elements of $K_{0} A$ and $K_{1} A$ with traces on $R / I^{m}$ and traces on $I^{m}$ respectively; cf. [Q2, II, §2].

First we note that composing our map $K_{0} A \rightarrow H_{+}(\widehat{\mathscr{X}}(R, I))$ with the map on homology induced by the surjection $\widehat{\mathscr{X}}(R, I) \rightarrow X\left(R / I^{m}\right)$ gives a map

$$
K_{0} A \rightarrow H_{+}\left(X\left(R / I^{m}\right)\right) \subset H C_{0}\left(R / I^{m}\right) .
$$

Now a trace $\tau$ on the algebra $R / I^{m}$ is the same as a linear functional on $H C_{0}\left(R / I^{m}\right)$. Composing $\tau$ with (7) gives the linear functional on $K_{0} A$ sending [e] to $\tau(\operatorname{tr}(\tilde{e}))$, where $\tilde{e}$ is a lift of $e$ to an idempotent over $R / I^{m}$. This effectively identifies (7) with the index pairing between elements of $K_{0} A$ and traces on $R / I^{m}$.

Similarly we have a map

$$
K_{1} A \rightarrow H_{-}\left(X\left(R / I^{m}\right)\right)=H C_{1}\left(R / I^{m}\right)
$$

so a cyclic cohomology class of degree one on $R / I^{m}$ determines a linear functional on $K_{1} A$. If the class is represented by the cyclic 1-cocycle $f$ on $R / I^{m}$, then the linear functional sends $[g] \in K_{1} A$ to $\operatorname{tr} f\left(p^{-1}, p\right)$, where $p$ is a lift of $g$ to $R / I^{m}$.

Let us now consider a trace $\tau$ on $I^{m}$ considered as an $R$-bimodule, that is, a linear functional on $I^{m}$ vanishing on $\left[R, I^{m}\right]$. Then $\tau$ determines a class in $H C^{1}\left(R / I^{m}\right)$ as follows. Extend $\tau$ to a linear functional $\tilde{\tau}$ on $R$. Then 
$(b \tilde{\tau})\left(r_{0}, r_{1}\right)=\tilde{\tau}\left(\left[r_{0}, r_{1}\right]\right)$ is a cyclic 1-cocycle on $R$ which vanishes if either $r_{0}$ or $r_{1}$ is in $I^{m}$. Hence it descends to give a cyciic 1-cocycle

$$
f\left(r_{0}+I^{m}, r_{1}+I^{m}\right)=\tilde{\tau}\left(\left[r_{0}, r_{1}\right]\right)
$$

on $R / I^{m}$. A different choice of extension changes this cyclic cocycle by $b h$, where $h$ is a linear functional on $R / I^{m}$, so we obtain a well-defined class $[\tau] \in H C^{1}\left(R / I^{m}\right)$.

Let us write $\langle[\tau],[g]\rangle$ for the value on $[g]$ of the linear functional on $K_{1} A$ associated to the class $[\tau]$. We now compute this number.

Let $p, q$ be liftings of $g, g^{-1}$ respectively to matrices over $R$, and let $x=1-q p, y=1-p q$, so that $x, y$ have entries in $I$. Note that $q y=x q$, hence $q y^{n}=x^{n} q$ for all $n$. The matrix

$$
q_{m}=\sum_{n=0}^{m-1} q y^{n}=\sum_{n=0}^{m-1} x^{n} q
$$

satisfies $p q_{m}=\sum_{n=0}^{m-1}(1-y) y^{n}=1-y^{m}$ and similarly $q_{m} p=1-x^{m}$. Thus $q_{m}$ is an inverse for $p \bmod I^{m}$ and we have

$$
1-p q_{m}=(1-p q)^{m}, \quad 1-q_{m} p=(1-q p)^{m} .
$$

Writing $p+I^{m}$ for the residue class of $p$ modulo matrices of the appropriate size over $I^{m}$, we have

$$
\begin{aligned}
\langle[\tau],[g]\rangle & =\operatorname{tr} f\left(\left(p+I^{m}\right)^{-1}, p+I^{m}\right)=\operatorname{tr} f\left(q_{m}+I^{m}, p+I^{m}\right) \\
& =\operatorname{tr} \tilde{\tau}\left(\left[q_{m}, p\right]\right)=\operatorname{tr} \tau\left(q_{m} p-p_{m} q\right) \\
& =\operatorname{tr} \tau\left(1-p q_{m}\right)-\operatorname{tr} \tau\left(1-q_{m} p\right) \\
& =\operatorname{tr} \tau\left((1-p q)^{m}\right)-\operatorname{tr} \tau\left((1-q p)^{m}\right) .
\end{aligned}
$$

This shows that up to sign $\langle[\tau],[g]\rangle$ is the index of $[g]$ with respect to $\tau$ in analogy with the index theory of pseudo-differential operators. Thus the index pairing between elements of $K_{1} A$ and traces on $I^{m}$ can be obtained from (8).

In principle the families of maps (7) and (8) for different $m$ contain less information than the maps (3) involving $\widehat{\mathscr{X}}(R, I)$ because of the Milnor exact sequence for the homology of a tower of supercomplexes.

The universal extension. For the universal extension we know that the homology of $\widehat{\mathscr{X}}(R A, I A)$ is the periodic cyclic homology, hence (3) yields canonical maps

$$
K_{i} A \rightarrow H P_{i+2 \mathbb{Z}} A, \quad i=0,1 .
$$

We now apply our discussion to compute these maps and identify them with the Chern character maps in cyclic homology theory.

Let $\widehat{R} A$ be the $I A$-adic completion of $R A$, and note that it can be identified with $\widehat{\Omega}^{+} A$ equipped with the Fedosov product. Similarly we can identify $M_{k} \widehat{R} A$ with $M_{k} \widehat{\Omega}^{+} A$ equipped with the Fedosov product.

Given an idempotent matrix $e$ over $A$, we consider the obvious lifting of it to a matrix over $\widehat{R} A$ given by the inclusion $A \subset \widehat{\Omega}^{+} A$, and then apply 12.2 
to obtain an idempotent matrix. Since $e-e \circ e=d e^{2}$ is closed, and Fedosov product coincides with ordinary product when one of the forms is closed, we obtain the matrix

$$
\tilde{e}=e+\sum_{n \geq 1} \frac{(2 n) !}{(n !)^{2}}\left(e-\frac{1}{2}\right) d e^{2 n} \in M_{k} \widehat{\Omega}^{+} A
$$

which is idempotent for the Fedosov product and is a lifting of $e$ over $A$. The trace of this matrix

$$
\operatorname{tr}(\tilde{e})=\operatorname{tr}(e)+\sum_{n \geq 1} \frac{(2 n) !}{(n !)^{2}} \operatorname{tr}\left(\left(e-\frac{1}{2}\right) d e^{2 n}\right) \in \widehat{\Omega}^{+} A
$$

is then an even cycle in $\widehat{\mathscr{X}}(R A, I A)$, whose homology class represents the image of $[e] \in K_{0} A$ under (3) in the case of the universal extension. Thus by 6.2 and the following remark we know that upon applying the homotopy equivalence $c P$ (or $c P_{1,-1}$ ) to this cycle we obtain the $b+B$ cycle representing the image of $[e]$ in $H P_{+} A$. Now

$$
\kappa\left(\operatorname{tr}\left(\left(e-\frac{1}{2}\right) d e^{2 n}\right)\right)=-\operatorname{tr}\left(d e\left(e-\frac{1}{2}\right) d e^{2 n-1}\right)=\operatorname{tr}\left(\left(e-\frac{1}{2}\right) d e^{2 n}\right)
$$

since dee $=(1-e) d e$. Hence $\operatorname{tr}(\tilde{e})$ is $\kappa$-invariant, so $c P$ carries (11) to the $\kappa$-invariant $b+B$ cycle

$$
\operatorname{tr}(e)+\sum_{n \geq 1}(-1)^{n} \frac{(2 n) !}{(n !)} \operatorname{tr}\left(\left(e-\frac{1}{2}\right) d e^{2 n}\right) \in \widehat{\Omega}^{+} A
$$

representing the class in $H P_{+} A$ corresponding to [e] under (9).

Given an invertible matrix $g$ over $A$, we lift it in the obvious way to the matrix $g$ over $\widehat{R} A$, which by 12.3 must be invertible. Let $g^{[-1]}$ denote the inverse, that is, the inverse of $g$ with respect to the Fedosov product. We can compute this inverse by (4) using $p=g$ and $q=g^{-1}$ considered as matrices over $\widehat{R} A$. As $1-g \circ g^{-1}=d g d g^{-1}$ is closed, we find that the inverse of $g$ with respect to the Fedosov product is

$$
g^{[-1]}=\sum_{n \geq 0} g^{-1}\left(d g d g^{-1}\right)^{n} .
$$

The class in $H_{-}(\widehat{\mathscr{X}}(R A, I A))$ corresponding to $[g] \in K_{1} A$ is represented by the odd cycle which is the image of $\operatorname{tr}\left(g^{[-1]} \delta g\right) \in \Omega^{1}(\widehat{R} A)_{\natural}$ in

$$
\stackrel{\lim }{\longleftarrow} \Omega^{1}\left(R A / I A^{n}\right)_{\natural} \simeq \widehat{\Omega}^{-} A \text {. }
$$

Using $\downarrow(x \delta a)=x d a$ we see that this odd cycle is

$$
\sum_{n \geq 0} \operatorname{tr}\left(g^{-1}\left(d g^{-1} d g\right)^{n} d g\right)=\sum_{n \geq 0} \operatorname{tr}\left(g^{-1} d g\left(d g^{-1} d g\right)^{n}\right)
$$

which we know is killed by the differential $\beta$. Now

$$
\begin{aligned}
\kappa\left(\operatorname{tr}\left(g^{-1} d g\left(d g^{-1} d g\right)^{n}\right)\right) & =\operatorname{tr}\left(d g g^{-1} d g\left(d g^{-1} d g\right)^{n-1} d g^{-1}\right) \\
& =-\operatorname{tr}\left(g d g^{-1}\left(d g d g^{-1}\right)^{n}\right)
\end{aligned}
$$


so (14) is invariant under $\kappa^{2}$. Applying $c P_{1,-1}$ we obtain the $\kappa^{2}$-invariant $b+B$ cycle

$$
\sum_{n \geq 0}(-1)^{n} n ! \operatorname{tr}\left(g^{-1} d g\left(d g^{-1} d g\right)^{n}\right)
$$

representing the image of $[g]$ in $H P_{-} A$. Averaging then gives the homologous $\kappa$-invariant $b+B$ cycle

$$
\frac{1}{2} \sum_{n \geq 0}(-1)^{n} n ! \operatorname{tr}\left(g^{-1} d g\left(d g^{-1} d g\right)^{n}-g d g^{-1}\left(d g d g^{-1}\right)^{n}\right) .
$$

We summarize this discussion as follows.

Proposition 12.4. (1) Given an idempotent matrix $e$ over $A$, (10) is then an idempotent matrix with respect to the Fedosov product which is a lifting of $e$. The even form (12) is a $\kappa$-invariant $b+B$ cycle representing the image of $[e]$ under the canonical map (9) from $K_{0} A$ to $H P_{+} A$.

(2) Given an invertible matrix $g$ over $A,(13)$ is then the inverse of $g$ with respect to the Fedosov product. The odd forms (15) and (16) are respectively $\kappa^{2}$-invariant and $\kappa$-invariant cycles which represent the image of $[g]$ under the canonical map from $K_{1} A$ to $H P_{-} A$.

This result shows that the maps (9) we have constructed coincide with the usual Chern character maps; cf. [K3, $\S 9,10]$.

\section{Commutative Algebras}

The purpose of this section is to discuss special features of the above theory in the case of a commutative algebra.

Let us recall that for any algebra $A$ we have the space $\Omega A$ of its noncommutative differential forms, which comes equipped with a canonical DG algebra structure and operators $d, b, \kappa, B, P$. In particular, we have the mixed complex $(\Omega A, b, B)$ giving rise to the cyclic type homology of $A$. Furthermore we have the algebra $R A$ and supercomplex $X(R A)$, which by $\S 5$ can be constructed from $\Omega A$ and its structure.

Suppose now that $A$ is commutative. Then we also have the ordinary de Rham complex $\Omega_{A}$ considered in algebraic geometry. This may be defined abstractly as the universal (super) commutative DG algebra generated by $A$, and it may be constructed concretely as the exterior algebra over $A$ of the module $\Omega_{A}^{1}$ of Kähler differentials. Let

$$
\mu: \Omega A \rightarrow \Omega_{A}
$$

be the unique homomorphism of DG algebras extending the identity on $A$ in degree zero. Then $\mu$ is surjective and it identifies $\Omega_{A}$ with the quotient of $\Omega A$ by the ideal generated by supercommutators $[\omega, \eta]$.

We next observe that the operators $d, b, \kappa, B, P$ on $\Omega A$ descend to $\Omega_{A}$. Indeed, $d$ descends to the differential $d$ of $\Omega_{A}$, and $b$ descends to the zero operator by $\S 3(2)$. The rest are generated from $d$ and $b$, so they descend to give the operators

$$
b=0, \quad \kappa=1, \quad B=N d, \quad P=1, \quad \text { on } \Omega_{A}
$$


as is easily checked. In particular, putting $\tilde{\mu}=(N !)^{-1} \mu$, where $N$ ! is the scaling operator which multiplies by $n$ ! on $\Omega_{A}^{n}$, we have a surjection of mixed complexes

$$
\tilde{\mu}:(\Omega A, b, B) \rightarrow\left(\Omega_{A}, 0, d\right) .
$$

To simplify matters, we will assume

$$
\Omega_{A}^{n}=0, \quad n \gg 0 .
$$

This holds if $A$ is essentially of finite type, for smooth algebras [L, Appendix E] in particular. One can avoid this assumption by working appropriately with the truncated algebra $\Omega_{A} / \Omega_{A}^{>n}$ and completion $\widehat{\Omega}_{A}$.

From (3) we then obtain a surjection of supercomplexes

$$
\tilde{\mu}:(\widehat{\Omega} A, b+B) \rightarrow\left(\Omega_{A}, d\right)
$$

whence a canonical map

$$
H P_{\nu} A \rightarrow H_{\nu}\left(\Omega_{A}, d\right)
$$

from periodic cyclic homology to $(\mathbb{Z} / 2)$-graded de Rham cohomology. When $A$ is smooth the theorem of Hochschild-Kostant-Rosenberg [HKR], [L, 3.4.4] says that (3) is a quasi-isomorphism of mixed complexes (i.e., with respect to the differential $b$ ). As a consequence one deduces that the map (6) is an isomorphism.

We turn next to the analogues of $R A$ and $X(R A)$ that arise when we carry out the constructions of $\S 5$ using $\Omega_{A}$ in place of $\Omega A$. Let $R_{A}$ be the algebra given by the space $\Omega_{A}^{+}$equipped with the Fedosov product. This algebra was originally constructed in the case of differential forms on a manifold by Fedosov [F] in his version of the index theorem. There is a surjective homomorphism

$$
\mu: R A \rightarrow R_{A}
$$

given by (1), and $R_{A}$ is a nilpotent extension of $A$ by the assumption (4).

There are two possible analogues of $X(R A)$. On one hand we have $X\left(R_{A}\right)$, and on the other hand we can mimic the description of $X(R A)$ in terms of $\Omega A$ given in 5.5 to obtain the supercomplex $\left(\Omega_{A}, \beta \oplus \delta\right)$ where

$$
\beta=-2 d: \Omega_{A}^{-} \rightarrow \Omega_{A}^{+}, \quad \delta=N d: \Omega_{A}^{+} \rightarrow \Omega_{A}^{-} .
$$

Using (2) we see that the Hochschild 1-cocycle 5.3 on $R A$ with values in $\Omega^{-} A$ descends to the bilinear map

$$
(x, y) \mapsto|y| d(x y)+x d y
$$

from $R_{A}$ to $\Omega_{A}^{-}$, hence this must be a Hochschild 1-cocycle. Furthermore, it is clear we have a canonical surjection of supercomplexes

$$
X\left(R_{A}\right) \rightarrow\left(\Omega_{A}, \beta \oplus \delta\right)
$$

given by the identity in even degree and the map $\Omega^{1}\left(R_{A}\right)_{\natural} \rightarrow \Omega_{A}^{-}$corresponding to the Hochschild 1-cocycle (8). 
Composing this map with the scaling isomorphisms

$$
\left(\Omega_{A}, \beta \oplus \delta\right) \stackrel{c}{\rightarrow}\left(\Omega_{A}, N d\right) \stackrel{(N !)^{-1}}{\longrightarrow}\left(\Omega_{A}, d\right)
$$

where $c$ is as in 6.1 , we obtain the bottom arrow $\alpha$ of the square

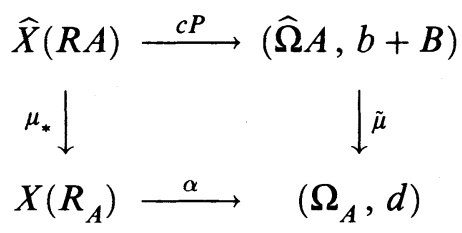

which is easily seen to be commutative; here $\widehat{X}(R A)=\widehat{\mathscr{X}}(R A, I A)$ and $c P$ is a homotopy equivalence by 6.2 .

When $A$ is smooth $\tilde{\mu}$ is a quasi-isomorphism, hence $\mu: R A \rightarrow R_{A}$ induces an injection $H P_{\nu} A \rightarrow H_{\nu}\left(X\left(R_{A}\right)\right)$. Formulating this dually we have proved

Proposition 13.1. If $A$ is smooth, then every periodic cyclic cohomology class of $A$ comes from a trace or cycle 1-cocycle on the nilpotent extension $R_{A}$.

Remarks. (1) Given an arbitrary nonnegatively graded commutative DG algebra $\Omega$, let $R$ be the algebra of its even elements under Fedosov product. The pairing defined by $(8)$ is then a 1-cocycle on $R$, and one has a map of supercomplexes

$$
\alpha: X(R) \rightarrow(\Omega, d)
$$

generalizing the map in (10).

(2) Although it will not be needed, we mention the calculation of $\Omega^{1}\left(R_{A}\right)_{\natural}$ in terms of differential forms. Define the operator

$$
\partial: \Omega_{A}^{n} \rightarrow \Omega_{A}^{n-1} \otimes_{A} \Omega_{A}^{1}
$$

by

$$
\partial\left(\xi_{1} \cdots \xi_{n}\right)=\sum_{j=1}^{n}(-1)^{j-1} \xi_{1} \cdots \widehat{\xi}_{j} \cdots \xi_{n} \otimes \xi_{j}
$$

where the $\xi_{j}$ are 1-forms. For $x, y \in \Omega_{A}^{+}$put

$$
\psi(x, y)=x \partial(d y)-d x \partial y \in \Omega_{A}^{+} \otimes_{A} \Omega_{A}^{1} .
$$

One can show that $\psi$ is a 1-cocycle with respect to Fedosov product on even forms, and that it induces an isomorphism

$$
\Omega^{1}\left(R_{A}\right)_{\natural} \stackrel{\sim}{\rightarrow} \Omega_{A}^{+} \otimes_{A} \Omega_{A}^{1}, \quad \natural(x \delta y) \mapsto \psi(x, y)
$$

with inverse $x \otimes d a \mapsto \mathfrak{t}(x \delta a)$.

Finally we consider the composition of the Chern character from $K_{0} A, K_{1} A$ to periodic cyclic homology with the canonical map (6) from the latter to $(\mathbb{Z} / 2)$ graded de Rham cohomology. Applying $\tilde{\mu}$ to the $b+B$ cycles $\S 12$ (12) and (15) we obtain the closed forms in the de Rham complex

$$
\sum_{n \geq 0} \frac{(-1)^{n}}{n !} \operatorname{tr}\left(e d e^{2 n}\right),
$$




$$
\sum_{n \geq 0} \frac{n !}{(2 n+1) !} \operatorname{tr}\left(\left(g^{-1} d g\right)^{2 n+1}\right)
$$

where we have used that $\operatorname{tr}\left(d e^{2 n}\right)=0$ for $n>0$ by virtue of the supercommutativity of $\Omega_{A}$. These agree with the usual Chern character forms associated to $e, g$ except for the extra signs $(-1)^{n}$ in (11) and the missing signs $(-1)^{n}$ in (12).

Ignoring these signs for the moment, let us consider the construction of the Chern character given in $\S 12$ in light of the square (10). To obtain the Chern character in de Rham cohomology it suffices to lift $e, g$ into $R_{A}$, take the corresponding cycles in $X\left(R_{A}\right)$, then map to the de Rham complex by $\alpha$. This unusual construction goes back to Fedosov's version $[F]$ of the index theorem.

The signs we have mentioned result from the fact that we use the differential $b+B$ in the supercomplex defining periodic cycle homology. If instead we use $-b+B$, the signs $(-1)^{n}$ occurring in $\S 12(12)$ and (15) are absent, and we obtain the usual Chern character forms in the de Rham complex. This observation suggests that it might have been better from the viewpoint of signs if from the outset we had defined the supercomplexes associated to a mixed complex, e.g., $\Omega R / F^{n} \Omega R$ and $X(R)$ using the differential $B-b$.

\section{TENSOR PRODUCTS}

In this section we examine the behavior of the $X$ complex when we form the tensor product $S \otimes T$ of two algebras $S$ and $T$. We wish to compare $X(S \otimes T)$ with the tensor product of the supercomplexes $X(S)$ and $X(T)$. It turns out that $X(S) \otimes X(T)$ lies intermediate between $X(S \# T)$ and $X(S \otimes T)$, where $S \# T$ is a certain square-zero extension of $S \otimes T$.

Let $x$ 's denote elements of $S$, and let $y$ 's denote elements of $T$. Let $F=S * T$ be the free product algebra, and let $J=F[S, T] F$ be the ideal generated by all commutators $[x, y]$. The quotient algebra $F / J$ can then be identified with the tensor product algebra $S \otimes T$ in such a way that $x \otimes 1$ and $1 \otimes y$ correspond to the congruence classes of $x$ and $y$ modulo $J$. We define $S \# T$ to be the quotient algebra $F / J^{2}$.

In [CQ1, 1.4] we gave a Fedosov type description of $F$ analogous to the ones for $R A$ and $Q A$. This description to first order says there is a vector space isomorphism

$$
S \otimes T \oplus \Omega^{1} S \otimes \Omega^{1} T \stackrel{\sim}{\rightarrow} F / J^{2}
$$

given by $x \otimes y \mapsto x y, x_{0} d x_{1} \otimes y_{0} d y_{1} \mapsto x_{0} y_{0}\left[x_{1}, y_{1}\right]$. Moreover, the product on the left side corresponding to the product in $F / J^{2}$ is given by

$$
\left(x_{0} \otimes y_{0}\right) \circ\left(x_{1} \otimes y_{1}\right)=x_{0} x_{1} \otimes y_{0} y_{1}-x_{0} d x_{1} \otimes d y_{0} y_{1}
$$

together with the obvious left and right multiplication of $S \otimes T$ on $\Omega^{1} S \otimes \Omega^{1} T$. It is easy to check (2) directly, namely, we have

$$
\begin{aligned}
\left(x_{0} y_{0}\right)\left(x_{1} y_{1}\right) & =x_{0} x_{1} y_{0} y_{1}-x_{0}\left[x_{1}, y_{0}\right] y_{1} \\
& =x_{0} x_{1} y_{0} y_{1}-x_{0}\left[x_{1}, y_{0} y_{1}\right]+x_{0} y_{0}\left[x_{1}, y_{1}\right]
\end{aligned}
$$


in $F / J^{2}$, and the corresponding element in $S \otimes T \oplus \Omega^{1} S \otimes \Omega^{1} T$ is $x_{0} x_{1} \otimes y_{0} y_{1}-x_{0} d x_{1} \otimes d\left(y_{0} y_{1}\right)+x_{0} d x_{1} \otimes y_{0} d y_{1}=x_{0} x_{1} \otimes y_{0} y_{1}-x_{0} d x_{1} \otimes d y_{0} y_{1}$ as claimed.

We now consider the tensor product $X(S) \otimes X(T)$ of the supercomplexes $X(S)$ and $X(T)$. To simplify the notation we suppress the $\boxminus$ symbol used previously for the canonical map to the commutator quotient space, so that $x_{0} d x_{1}$ now stands for an element of $\Omega^{1} S$ or $\Omega^{1} F$, or its image in some quotient space of either of these, which will be clear from the context. Similarly we write $b, d$ instead of $\bar{b}, \downarrow d$ for the differentials in the $X$ complex. Then $X(S) \otimes X(T)$ is the supercomplex

$$
\begin{array}{ccc}
S \otimes T & & \Omega^{1} S_{\natural} \otimes T \\
\oplus & \stackrel{\partial_{1}}{\leftrightarrows} & \oplus \\
\Omega^{1} S_{\natural} \otimes \Omega^{1} T_{\natural} & & S \otimes \Omega^{1} T_{\natural}
\end{array}
$$

where

$$
\partial_{0}=\left(\begin{array}{cc}
d \otimes 1 & -1 \otimes b \\
1 \otimes d & b \otimes 1
\end{array}\right), \quad \partial_{1}=\left(\begin{array}{cc}
b \otimes 1 & 1 \otimes b \\
-1 \otimes d & d \otimes 1
\end{array}\right) .
$$

We now show $X(S) \otimes X(T)$ is isomorphic to $\mathscr{X}^{2}(F, J)$, which we recall is the following quotient of $X(F)$ :

$$
F / J^{2}+[J, F] \leftrightarrows \Omega^{1} F /\left[\Omega^{1} F, F\right]+J \Omega^{1} F
$$

Proposition 14.1. One has an isomorphism of supercomplexes $\phi: X(S) \otimes X(T) \stackrel{\sim}{\rightarrow}$ $\mathscr{X}^{2}(F, J)$ given by

$$
\begin{aligned}
& \phi(x \otimes y)=x y, \\
& \phi\left(x_{0} d x_{1} \otimes y_{0} d y_{1}\right)=x_{0} y_{0}\left[x_{1}, y_{1}\right], \\
& \phi\left(x_{0} d x_{1} \otimes y\right)=x_{0} d x_{1} y, \\
& \phi\left(x \otimes y_{0} d y_{1}\right)=x y_{0} d y_{1}
\end{aligned}
$$

where the elements on the right are to be interpreted as the images in $\mathscr{X}^{2}(F, J)$ of the indicated elements of $F$ and $\Omega^{1} F$.

Proof. As (1) gives an isomorphism of $\Omega^{1} S \otimes \Omega^{1} T$ with $J / J^{2}$ as bimodules over $S \otimes T=F / J$, it induces an isomorphism

$$
S \otimes T \oplus \Omega^{1} S_{\natural} \otimes \Omega^{1} T_{\natural} \stackrel{\sim}{\rightarrow} F / J^{2}+[J, F] .
$$

This shows $\phi$ is an isomorphism in even degree.

Next, because $F$ is the free product $S * T$, the inclusion homomorphisms from $S$ and $T$ to $F$ induce a canonical $F$-bimodule isomorphism

$$
F \otimes_{S} \Omega^{1} S \otimes_{S} F \oplus F \otimes_{T} \Omega^{1} T \otimes_{T} F \stackrel{\sim}{\rightarrow} \Omega^{1} F .
$$

This follows easily from the universal properties of the free product and $\Omega^{1}$. Tensoring on both sides with $F / J$ gives an isomorphism of $(S \otimes T)$-bimodules

$$
T \otimes \Omega^{1} S \otimes T \oplus S \otimes \Omega^{1} T \otimes S \stackrel{\sim}{\rightarrow} \Omega^{1} F / J \Omega^{1} F+\Omega^{1} F J
$$


such that $y_{0} \otimes x_{0} d x_{1} \otimes y_{1} \mapsto y_{0} x_{0} d x_{1} y_{1}$, and similarly on the other summand. Identifying the commutator quotient spaces as

$$
\begin{array}{ll}
\left(T \otimes \Omega^{1} S \otimes T\right)_{\natural} \stackrel{\sim}{\rightarrow} \Omega^{1} S_{\natural} \otimes T, & y_{0} \otimes x_{0} d x_{1} \otimes y_{1} \mapsto x_{0} d x_{1} \otimes y_{1} y_{0}, \\
\left(S \otimes \Omega^{1} T \otimes S\right)_{\natural} \stackrel{\sim}{\rightarrow} S \otimes \Omega^{1} T_{\natural}, & x_{0} \otimes y_{0} d y_{1} \otimes x_{1} \mapsto x_{1} x_{0} \otimes y_{0} d y_{1},
\end{array}
$$

we obtain an isomorphism

$$
\Omega^{1} S_{\natural} \otimes T \oplus S \otimes \Omega^{1} T_{\natural} \stackrel{\sim}{\rightarrow} \Omega^{1} F /\left[\Omega^{1} F, F\right]+J \Omega^{1} F
$$

which is the map $\phi$ in odd degree.

Thus $\phi$ is an isomorphism, and it remains only to check that it is compatible with the differentials. Let $\beta, \delta$ denote the $b, d$ differentials in $\mathscr{X}^{2}(F, J)$. We have

$$
\begin{aligned}
\beta \phi\left(x_{0} d x_{1} \otimes y\right) & =\beta\left(x_{0} d x_{1} y\right)=\left[y x_{0}, x_{1}\right] \\
& =\left[x_{0} y, x_{1}\right]=x_{0}\left[y, x_{1}\right]+\left[x_{0}, x_{1}\right] y \\
& =\phi\left(-x_{0} d x_{1} \otimes d y+\left[x_{0}, x_{1}\right] \otimes y\right) \\
& =\phi \partial_{1}\left(x_{0} d x_{1} \otimes y\right)
\end{aligned}
$$

where we have used that $\left[\left[x_{0}, y\right], x_{1}\right] \in[J, F]$ vanishes in $\mathscr{X}^{2}(F, J)$. Also

$$
\begin{aligned}
\delta \phi\left(x_{0} d x_{1} \otimes y_{0} d y_{1}\right) & =d\left(x_{0} y_{0}\left[x_{1}, y_{1}\right]\right) \\
& =x_{0} y_{0}\left[d x_{1}, y_{1}\right]+x_{0} y_{0}\left[x_{1}, d y_{1}\right] \\
& =x_{0} d x_{1}\left[y_{1}, y_{0}\right]+\left[x_{0}, x_{1}\right] y_{0} d y_{1} \\
& =\phi\left(-x_{0} d x_{1} \otimes\left[y_{0}, y_{1}\right]+\left[x_{0}, x_{1}\right] \otimes y_{0} d y_{1}\right) \\
& =\phi \partial_{0}\left(x_{0} d x_{1} \otimes y_{0} d y_{1}\right)
\end{aligned}
$$

where we have

and

$$
x_{0} y_{0} d x_{1} y_{1}=y_{0} x_{0} d x_{1} y_{1}=x_{0} d x_{1} y_{1} y_{0}
$$

$$
x_{0} y_{0} y_{1} d x_{1}=y_{0} y_{1} x_{0} d x_{1}=x_{0} d x_{1} y_{0} y_{1}
$$

because a differential form containing a factor in $[S, T]$ vanishes in $\mathscr{X}^{2}(F, J)$. The other cases are easily verified.

Remarks. (1) This result implies the Kunneth formula

$$
H P(S \otimes T)=H P(S) \otimes H P(T)
$$

when $S$ and $T$ are quasi-free. Indeed, in this situation $F$ is quasi-free [CQ1, 5.3], so the tower $\mathscr{X}(F, J)$ represents the cyclic homology type of $S \otimes T$. On the other hand, $S \otimes T$ has Hochschild cohomological dimension $\leq 2$, so the canonical surjection of $\widehat{\mathscr{X}}(F, J)$ onto $\mathscr{X}^{2}(F, J)$ is a quasi-isomorphism and $\mathscr{X}^{2}(F, J)$ calculates $H P(S \otimes T)$. Since $X(S)$ calculates $H P(S)$ and similarly for $T$, the desired formula follows from the proposition.

(2) The isomorphism $\phi$ is clearly natural (i.e., a morphism of functors of $S$ and $T$ ), but it is not completely canonical, as it depends upon the order of the pair of algebras $S, T$ in the following way. Let

$$
\phi_{S T}: X(S) \otimes X(T) \stackrel{\sim}{\rightarrow} \mathscr{X}^{2}(F, J)
$$


denote the isomorphism just constructed. One can compare $\phi_{S T}$ with $\phi_{T S} \sigma$, where

$$
\sigma: X(S) \otimes X(T) \stackrel{\sim}{\rightarrow} X(T) \otimes X(S)
$$

is the commutativity isomorphism for the tensor product of supercomplexes. One finds that $\phi_{S T}^{-1} \phi_{T S} \sigma$ is the identity on the subspaces $\Omega^{1} S_{\natural} \otimes \Omega^{1} T_{\natural}, \Omega^{1} S_{\natural} \otimes T$ and $S \otimes \Omega^{1} T_{\natural}$, but on $S \otimes T$ one has

$$
\phi_{S T}^{-1} \phi_{T S} \sigma(x \otimes y)=x \otimes y-d x \otimes d y
$$

so $\phi_{S T} \neq \sigma_{T S} \sigma$.

We now use the preceding proposition and the canonical surjections

$$
X(S \# T)=X\left(F / J^{2}\right) \rightarrow \mathscr{X}^{2}(F, J) \rightarrow X(F / J)=X(S \otimes T)
$$

to obtain natural surjections

$$
X(S \# T) \stackrel{f}{\rightarrow} X(S) \otimes X(T) \stackrel{g}{\rightarrow} X(S \otimes T)
$$

such that $g f$ is the map on $X$ supercomplexes induced by the canonical surjection $\pi: S \# T \rightarrow S \otimes T$. Note that $g$ is given by the formulas (3) where the expressions on the right are to be interpreted as elements of $X(S \otimes T)$. Like $\phi$ the map $f$ depends on the ordering of the pair $S, T$. The map $g$ however is canonical, since the order ambiguity is killed by $g$.

Let $k: X(S \otimes T) \rightarrow S_{\natural} \otimes X(T)$ be the canonical surjection induced by the DG algebra homomorphism $\Omega(S \otimes T) \rightarrow S \otimes \Omega T$ extending the identity in degree zero, i.e.,

$$
\begin{aligned}
k(x \otimes y) & =\mathrm{\natural}(x) \otimes y, \\
k\left(\left(x_{0} \otimes y_{0}\right) d\left(x_{1} \otimes y_{1}\right)\right) & =\mathrm{\natural}\left(x_{0} x_{1}\right) \otimes y_{0} d y_{1} .
\end{aligned}
$$

One easily verifies that

$$
k g=\varepsilon \otimes 1: X(S) \otimes X(T) \rightarrow S_{\natural} \otimes X(T)
$$

where $\varepsilon: X(S) \rightarrow S_{\natural}$ is the canonical surjection between levels 1 and 0 of the Hodge tower of $\Omega S$; here $S_{\natural}$ is to be regarded as a supercomplex concentrated in even degree.

The following summarizes these facts.

Proposition 14.2. One has a commutative diagram

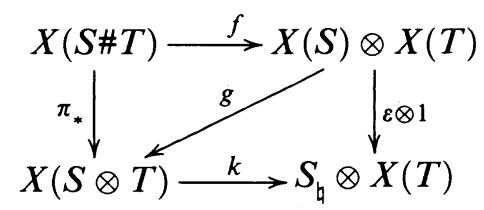

where the arrows are natural surjections of supercomplexes.

We now apply this result to study the homotopy behavior of the $X$ complex. 
Proposition 14.3. Let $A$ be a quasi-free algebra, and let $u: A \rightarrow S \otimes T$ be a homomorphism. Then $k u_{*}: X(A) \rightarrow S_{\natural} \otimes X(T)$ can be lifted to a map of supercomplexes $X(A) \rightarrow X(S) \otimes X(T)$.

Proof. As $S \# T$ is a square-zero extension of $S \otimes T$, we can lift $u$ to a homomorphism $u_{0}: A \rightarrow S \# T$. By 14.2 the composition

$$
X(A) \stackrel{u_{0 *}}{\longrightarrow} X(S \# T) \stackrel{f}{\longrightarrow} X(S) \otimes X(T) \stackrel{\varepsilon \otimes 1}{\longrightarrow} S_{\natural} \otimes X(T)
$$

gives the desired map.

To understand the significance of this result, consider the mapping supercomplex $\operatorname{Hom}(X(A), X(T))$ and note that $k u_{*}$ gives rise to a map of supercomplexes

$$
S_{\natural}^{*} \rightarrow \operatorname{Hom}(X(A), X(T))
$$

sending a trace $\tau: S_{\natural} \rightarrow \mathbb{C}$ to $(\tau \otimes 1) k u_{*}$. The proposition implies that if we regard $S_{\natural}^{*}$ embedded as a subcomplex of $X(S)^{*}$ by the transpose of $\varepsilon$, then (5) extends to a map of supercomplexes

$$
X(S)^{*} \rightarrow \operatorname{Hom}(X(A), X(T)) .
$$

Consequently, if the trace $\tau$ is nullhomotopic, i.e., $\tau=\tau^{\prime} d$ for some trace $\tau^{\prime}$ on $\Omega^{1} S$, then the odd operator corresponding to $\tau^{\prime}$ under (6) is a nullhomotopy for the operator corresponding to $\tau$. This proves

Corollary 14.4. If the trace $\tau$ is nullhomotopic, then $(\tau \otimes 1) k u_{*}: X(A) \rightarrow X(T)$ is nullhomotopic.

As an example, consider $S=\mathbb{C}[t]$ with

$$
\tau(f(t))=f(1)-f(0), \quad \tau^{\prime}(f(t) d t)=\int_{0}^{1} f(t) d t .
$$

In this situation a homomorphism $A \rightarrow S \otimes T$ is a polynomial family of homomorphisms $u_{t}: A \rightarrow T$, and the map from $X(A)$ to $X(T)$ associated to $\tau$ is the difference of the induced maps $u_{1_{*}}, u_{0_{*}}$. The proposition says that these induced maps are homotopic when $A$ is quasi-free, which provides another proof of the second assertion in 8.1. With some more work one can obtain the actual Cartan homotopy formula of $\S 7$ by concretely constructing the lifting $u_{0}$ used above.

Let us now assume $S$ to be commutative. In this case we can think of $u$ as a family of homomorphisms from $A$ to $T$ parametrized by the spectrum of $S$.

We have $S=S_{\natural}, \Omega^{1} S_{\natural}=\Omega_{S}^{1}$, and $X(S)=\Omega_{S} / \Omega_{S}^{>1}$, where $\Omega_{S}^{>n}$ denotes the ideal in the ordinary de Rham complex consisting of forms of degree $>n$.

We shall prove the following higher order version of 14.3.

Proposition 14.5. With these assumptions the map $k u_{*}: X(A) \rightarrow S \otimes X(T)$ can be lifted to a map of supercomplexes

$$
X(A) \rightarrow \underset{n}{\lim }\left(\left(\Omega_{S} / \Omega_{S}^{>n}\right) \otimes X(T)\right) .
$$


Proof. Let $R_{n}$ be the algebra of even elements of $\Omega_{n}=\Omega_{S} / \Omega_{S}^{>n}$ equipped with Fedosov product; clearly $R_{2 k+1}=R_{2 k}$ and $R_{1}=R_{0}=S$. By $\S 13$ (9) there is a canonical map of supercomplexes

$$
\alpha: X\left(R_{n}\right) \rightarrow \Omega_{S} / \Omega_{S}^{>n}
$$

given by the identity in even degree, and $\downarrow(x \delta y) \mapsto|y| d(x y)+x d y$ in odd degree.

Now $R_{n}$ is a nilpotent extension of $S$, hence $R_{n} \otimes T$ is a nilpotent extension of $S \otimes T$, and so $R_{n} \# T$, which is a square-zero extension of $R_{n} \otimes T$, is also a nilpotent extension of $S \otimes T$. Thus we have a tower of nilpotent extensions $R_{n} \# T$ of $S \otimes T$, and as $A$ is quasi-free, there is a compatible family of homomorphisms $u_{n}: A \rightarrow R_{n} \# T$ lifting $u$. This gives a compatible family of maps of supercomplexes

$$
X(A) \stackrel{u_{n *}}{\longrightarrow} X\left(R_{n} \# T\right) \stackrel{f}{\longrightarrow} X\left(R_{n}\right) \otimes X(T) \stackrel{\alpha \otimes 1}{\longrightarrow}\left(\Omega_{S} / \Omega_{S}^{>n}\right) \otimes X(T)
$$

which starts with $k u_{*}$ when $n=0$, as one sees from the proof of 14.3 and the fact that $\alpha=\varepsilon$ for $n=0$. This proves the proposition.

As a consequence, we have a map of supercomplexes

$$
\bigoplus_{n \geq 0}\left(\Omega_{S}^{n}\right)^{*} \rightarrow \operatorname{Hom}(X(A), X(T))
$$

extending (5), which associates to any (finite dimensional) current $\eta$ an operator $\tilde{\eta}: X(A) \rightarrow X(T)$ of the same parity such that to the boundary of $\eta$ corresponds the operator $[d, \tilde{\eta}]$. In particular, a closed current gives rise to a map of supercomplexes of the same parity whose class modulo homotopy depends only on the homology class of the current.

\section{REFERENCES}

[Co1] A. Connes, Non-commutative differential geometry, Inst. Hautes Études Sci. Publ. Math. 62 (1985), 257-360.

[Co2] _ Entire cyclic cohomology of Banach algebras and characters of $\theta$-summable Fredholm modules, $K$-Theory 1 (1988), 519-548.

[CC] A. Connes and J. Cuntz, Quasi-homomorphisms, cohomologie cyclicque et positivité, Comm. Math. Phys. 114 (1988), 515-526.

[Cu1] J. Cuntz, Universal extensions and cyclic cohomology, C. R. Acad. Sci. Paris Sér. I Math. 309 (1989), 5-8.

[Cu2] _ Cyclic cohomology and K-homology, Proc. Internat. Congr. Math. (Kyoto, Japan 1990), Springer-Verlag, Berlin and New York, 1991.

[CQ1] J. Cuntz and D. Quillen, Algebra extensions and nonsingularity, J. Amer. Math. Soc. (to appear).

[CQ2] __, Operators on noncommutative differential forms and cyclic homology, Geometry, Topology, and Physics for Raoul Bott, International Press, Cambridge, MA, 1995.

[D] P. Deligne, Harvard lectures, fall 1969.

[F] B. V. Fedosov, Analytical formulas for the index of an elliptic operator, Trudy Moskov. Mat. Obshch. 30 (1974), 159-241; English transl., Trans. Moscow Math. Soc. 30 (1974), 159-240.

[G] T. G. Goodwillie, Cyclic homology and the free loopspace, Topology 24 (1985), 187-215. 
[H] R. Hartshorne, On the de Rham cohomology of algebraic varieties, Inst. Hautes Études Sci. Publ. Math. 45 (1975), 5-99.

[HKR] G. Hochschild, B. Kostant, and A. Rosenberg, Differential forms on regular affine algebras, Trans. Amer. Math. Soc. 102 (1962), 383-408.

[JK] J. D. S. Jones and C. Kassel, Bivariant cyclic theory, $K$-Theory 3 (1989), 339-365.

[Ka] M. Karoubi, Homologie cyclique et K-théorie, Astérisque 149 (1987).

[K1] C. Kassel, Cyclic homology, comodules, and mixed complexes, J. Algebra 107 (1987), 195-216.

[K2] _- Caractère de Chern bivariant, $K$-Theory 3 (1989), 367-400.

[K3] - Homologie cyclique, caractère de Chern et lemme de perturbation, J. Reine Angew. Math. 408 (1990), 159-180.

[L] J.-L. Loday, Cyclic homology, Springer-Verlag, Berlin and New York, 1992.

[LQ] J.-L. Loday and D. Quillen, Cyclic homology and the Lie algebra homology of matrices, Comment. Math. Helv. 59 (1984), 565-591.

[N] V. Nistor, $A$ bivariant Chern character for p-summable quasihomomorphisms, $K$-Theory 5 (1991), 193-211.

[Q1] D. Quillen, Algebra cochains and cyclic cohomology, Inst. Hautes Études Sci. Publ. Math. 68 (1989), 139-174.

[Q2] _- Cyclic cohomology and algebra extensions, $K$-Theory 3 (1989), 205-246.

[Q3] _ Bivariant cyclic cohomology and models for cyclic homology types, J. Pure Appl. Algebra (to appear).

Institute of Mathematics, University Of Heidelberg, Im Neuenheimer Feld 288, 69120 HeIDELBERG, GeRMANY

E-mail address: cuntz@math.uni-heidelberg.de

University of Oxford, Mathematical Institute, 24-29 St. Giles, Oxford OX1 3LB, UNITED KINGDOM

E-mail address: quillen@maths.ox.ac.uk 